\title{
A REPRESENTATION OF PARTIALLY ORDERED PREFERENCES ${ }^{1}$
}

\author{
By Teddy Seidenfeld, Mark J. Schervish and Joseph B. Kadane
}

\section{Carnegie Mellon University}

\begin{abstract}
This essay considers decision-theoretic foundations for robust Bayesian statistics. We modify the approach of Ramsey, de Finetti, Savage and Anscombe and Aumann in giving axioms for a theory of robust preferences. We establish that preferences which satisfy axioms for robust preferences can be represented by a set of expected utilities. In the presence of two axioms relating to state-independent utility, robust preferences are represented by a set of probability/utility pairs, where the utilities are almost state-independent (in a sense which we make precise). Our goal is to focus on preference alone and to extract whatever probability and/or utility information is contained in the preference relation when that is merely a partial order. This is in contrast with the usual approach to Bayesian robustness that begins with a class of "priors" or "likelihoods," and a single loss function, in order to derive preferences from these probability/utility assumptions.
\end{abstract}

\section{Introduction and overview.}

1.1. Robust Bayesian preferences. This essay is about decision-theoretic foundations for robust Bayesian statistics. The fruitful tradition of Ramsey (1931), de Finetti (1937), Savage (1954) and Anscombe and Aumann (1963) seeks to ground Bayesian inference on a normative theory of rational choice. Rather than accept the traditional probability models and loss functions as given, Savage is explicit about the foundations. He axiomatizes a theory of preference using a binary relation over acts, $A_{1} \leq A_{2}$, "act $A_{1}$ is not preferred to act $A_{2}$." Then, he shows that $\lesssim$ is represented by a unique personal probability (state-independent) utility pair according to subjective expected utility. That is, he shows there exists exactly one pair $(p, U)$ such that, for all acts $A_{1}$ and $A_{2}, A_{1} \leq A_{2}$ if and only if $E_{p, U}\left[A_{1}\right] \leq E_{p, U}\left[A_{2}\right]$. [More precisely, in Savage's theory what is needed to justify the assertion that $p$ is the agent's personal probability is the added assumption that each consequence has a constant value in each state. Unfortunately this is ineffa-

\footnotetext{
Received November 1992; revised June 1995.

${ }^{1}$ The research of Teddy Seidenfeld was supported by the Buhl Foundation and NSF Grant SES-92-08942. Mark Schervish's research on this project was supported through ONR Contract N00014-91-J-1024 and NSF Grant DMS-88-05676. Joseph Kadane's research was supported by NSF grants SES-89-0002 and DMS-90-05858, and ONR contract N00014-89-J-1851.

AMS 1991 subject classifications. Primary 62C05; secondary 62A15.

Key words and phrases. Robust statistics, axioms of decision theory, state-dependent utility, partial order.
} 
ble in Savage's language of preference over acts. [See Schervish, Seidenfeld and Kadane (1990)]. We discuss this in Section 4, below.

In recent years, either under the headings of Bayesian robustness [Berger (1985), Section 4.7; Hartigan (1983), Chapter 12; Kadane (1984)] or sensitivity analysis [Rios Insua (1990)] it has become an increasingly important issue to show how to arrive at Bayesian conclusions from logically weaker assumptions than are required by the traditional Bayesian theory. Given data and a particular likelihood from a statistical model, for example, how large a class of prior probabilities leads to a class of posterior probabilities that are in agreement about some event of interest? Our work differs from the common trend in Bayesian robustness in much the same way that Savage's work differs from the traditional use of probability models and loss functions in Bayesian decision theory. Our goal is to axiomatize robust preferences directly, rather than to robustify given statistical models. Results in this theory are strikingly different from those obtained in the existing Bayesian robustness literature.

For an illustration of the difference, suppose two Bayesian agents each rank the desirability of Anscombe-Aumann ("horse lottery") acts according to his /her subjective expected utility. ("Horse lotteries" are defined in Section 2.) Let $\left(p_{1}, U_{1}\right)$ and $\left(p_{2}, U_{2}\right)$ be the probability/utility pairs representing these two decision makers and assume they have different beliefs and values: that is, assume $p_{1} \neq p_{2}$ and $U_{1} \neq U_{2}$. Denote by $\prec_{1}$ and $\prec_{2}$ their respective (strict) preference relations, each a weak order over acts. Suppose now our goal is to find those coherent (Anscombe-Aumann) preference relations $\lesssim$ corresponding to probability/utility pairs $(p, U)$ such that the following Pareto condition applies:

If $E_{p_{1}, U_{1}}\left[A_{1}\right]<E_{p_{1}, U_{1}}\left[A_{2}\right]$ and $E_{p_{2}, U_{2}}\left[A_{1}\right]<E_{p_{2}, U_{2}}\left[A_{2}\right]$, then $E_{p, U}\left[A_{1}\right]<$ $E_{p, U}\left[A_{2}\right]$. In words, when both agents strictly prefer act $A_{2}$ to act $A_{1}$, then this shared preference is robust for all efficient, cooperative Bayesian decisions that the pair make together. [We assume that though the two Bayesian agents may discuss their individual preferences, nonetheless, some differences remain in their beliefs and in their values even after such conversations. See DeGroot (1974) for a rival model.] We have the following theorem.

Theorem 1 [Seidenfeld, Kadane and Schervish (1989)]. Assume there exists a pair of prizes $\left\{r_{*}, r^{*}\right\}$ which the two agents rank in the same order: $r_{*} \prec_{i} r^{*}(i=1,2)$. Then the set of probability/utility pairs, each of which satisfies the Anscombe-Aumann theory and each of which agrees with the strict preferences shared by these two decision makers, consists exactly of the two pairs themselves $\left\{\left(p_{1}, U_{1}\right),\left(p_{2}, U_{2}\right)\right\}$. There are no other coherent, Pareto compromises. [There is no coherent weak order meeting the strong Pareto condition, which requires that $A_{1} \prec A_{2}$ if $A_{1} \preccurlyeq_{i} A_{2}(i=1,2)$ and at least one of these two preferences is strict.]

Thus, with respect to Pareto-robust preferences, the set of probability/ utility pairs for the problem of two distinct Bayesians is not connected and 
therefore not convex. Hence, a common method of proof-separating hyperplanes (used to develop expected utility representations) - is not available in our investigation. This is just one way in which our methods differ from the usual robust Bayesian analysis. We want the strict preferences held in common by two Bayesians to be a special case of robust preferences. Applied to a class of weak orders, the Pareto condition creates a strict partial order $\prec$ : to wit, the binary relation $\prec$ is irreflexive and transitive.

Our view of robustness is that sometimes a person does not have a (strict) preference for act $A_{1}$ over act $A_{2}$ nor for $A_{2}$ over act $A_{1}$ nor are they indifferent options. Assume that strict preference is a transitive relation. Then such a person's preferences are modeled by a partial order. We ask, under what assumptions on this partial order is there a set of probability/utility pairs agreeing with it according to expected utility theory, a set which characterizes that partial order? In this we are exploring the possibility pointed out by Savage [(1954), page 21].

The general form of our inquiry is as follows. Axiomatize coherent preference $\prec$ as a partial order and establish a representation for it in terms of a set of probability/utility pairs. That is, we characterize each coherent, partially ordered preference $\prec$ in terms of the set of coherent weak orders $\{\lesssim\}$ that extend it. We rely on the usual expected utility theory to depict each coherent weak order $\precsim$ by one probability/utility pair $(p, U)$. Thus, we model $\prec$ by a set of probability/utility pairs.

In contrast with Savage's theory, which uses only personal probability, our approach is based on Anscombe-Aumann's "horse lottery" theory. Preferences over "horse lotteries" accommodate both personal and extraneous (agent-invariant) probabilities. Also, by characterizing strict preference in terms of a set of probability/utility pairs, we improve so-called one-way representations of, for example, Fishburn [(1982), Section 11], as we show more than existence of an agreeing probability/utility pair.

1.2. Overview. In outline, our approach is as follows: In Section 2 we introduce axioms for a partial order over Anscombe-Aumann horse lotteries (HL). Anscombe-Aumann theory contains three substantive axioms that incorporate the (von Neumann-Morgenstern) theory of cardinal utility for simple acts:

1. A postulate that preference $(\preceq)$ is a weak order-analogous to Savage's $\mathrm{P} 1$.

2. The independence postulate - analogous to Savage's P2, "sure thing."

3. An Archimedean condition, which plays an analogous role to Savage's P6.

Our replacement axioms for these are:

HL AxIOM 1. A postulate that strict preference $(\prec)$ is a strict partial order. 
HL Axiom 2. The independence postulate.

HL Axiom 3. A modified Archimedean axiom for discrete (not just simple) lotteries.

To avoid triviality, a commonplace assumption of expected utility theory is that not all acts are indifferent, for example, there exist two acts, $\mathbf{W}$ and $\mathbf{B}$ that do not satisfy $\mathbf{B} \precsim \mathbf{W}$. Let $\prec$ obey our (three) preference axioms on a domain of horse-lottery acts $\mathbf{H}_{\mathbf{R}}$. We show (Theorem 2 ) how to extend $\prec$ to a preference $\prec$ ' over a larger domain that includes two new acts $\mathbf{B}$ (best) and W (worst) where

$\left(\forall H_{1}, H_{2} \in \mathbf{H}_{\mathbf{R}}\right) \quad\left[\left(\mathbf{B} \prec^{\prime} H_{1} \prec^{\prime} \mathbf{W}\right) \&\left(H_{1} \prec H_{2}\right.\right.$ if and only if $\left.\left.H_{1} \prec^{\prime} H_{2}\right)\right]$.

Then we establish three related theorems (Theorems 3,4 and 5 ): $\prec$ is represented by a nonempty (maximal and convex) set $\mathscr{V}=\left\{V: \mathbf{H}_{\mathbf{R}} \rightarrow(0,1)\right\}$ of bounded, real-valued cardinal utilities $V(\cdot)$ defined for acts. Each $V \in \mathscr{V}$ induces a weak order $\precsim_{V}$ that agrees with $\prec$ on the domain of simple acts and almost agrees (Definition 10b) with $\prec$ on all acts. Moreover, given a set $\mathscr{Z}$ of bounded, real-valued cardinal (so-called) "linear" utilities, $Z(\cdot)$ defined on $\mathbf{H}_{\mathbf{R}}$, the partial order formed using the Pareto condition with the set $\mathscr{Z}$ satisfies our three axioms for preference.

In the light of the surprising "shape" that the family of agreeing subjective expected utilities can have (Theorem 1), we employ a modification of Szpilrajn's (1930) transfinite induction for extending a partial order. We show how to extend a partial order while preserving the other preference axioms. The proofs of all results appear in the Appendix. Also, we number definitions, lemmas, and corollaries to coincide with their logical order in our arguments, regardless of whether they appear for the first time in the body of the text or in the Appendix.

In Section 4 we turn our attention to the representation of $\mathscr{V}$ as a set of subjective expected utilities. We discuss when a linear utility $V$ over acts also is a subjective expected utility for a probability/utility pair $(p, U)$. Corollary 4.1 gives a representation of $\mathscr{V}$ in terms of sets of probability/statedependent utility pairs $\left(p,\left\{U_{j}: j=1, \ldots, n\right\}\right)$, where the utility $U_{j}(L)$ of a (von Neumann-Morgenstern) lottery $L$ may depend upon the accompanying state $s_{j}$. (This follows up the issue raised in the first paragraph in Section 1.1.) In Section 4.3 we introduce two axioms (HL Axioms 4 and 5) that parallel the fourth Anscombe-Aumann postulate. That postulate (and our replacements for it) permits a representation of preference using a (nearly) state-independent utility: where (with high personal probability) the value of a lottery $L$ does not depend (by more than amount $\varepsilon>0$ ) upon the state in which it is awarded. In Section 4.3 we lean heavily on the proof technique of Section 3 in order to find a representation for the partial order $\prec$ in terms of a set of agreeing pairs of probabilities and (nearly) state-independent utilities, Lemma 4.3 and Theorem 6. 
Section 5 is about conditional preference. Two theorems (Theorems 7 and 8) relate conditional (called-off) partially ordered preferences and Bayesian updating of the family of unconditional personal probabilities that agree with an unconditional partially ordered preference. We provide an example involving conditional probability that highlights the nonconvexity of the agreeing sets. In Section 6 we conclude with a review of several features that distinguish our results.

\section{The formal theory.}

2.1. The act space: a domain for the preference relation. We provide a representation for a partially ordered strict preference relation over (discrete) Anscombe-Aumann (1963) horse lotteries-acts that generalize von Neumann-Morgenstern (1947) lotteries to allow for uncertainty over states of nature.

Let $\mathbf{R}$ be a set of rewards. We develop our theory for countable sets $\mathbf{R}$.

Definition 1. A simple (von Neumann-Morgenstern) lottery is a simple probability distribution $P$ over $\mathbf{R}$, that is, a distribution with finite support. A discrete lottery is a countably additive probability over $R$ (with a countable support). Denote a lottery by $L$ and its distribution by $P$.

Horse lotteries are defined with respect to a finite partition of states. Let $\pi$ be a finite partition of the sure event $S$ into $n$ disjoint, mutually exhaustive nonempty (sets of) states, $\pi=\left\{s_{1}, \ldots, s_{n}: s_{i} \cap s_{j}=\varnothing\right.$ iff $i \neq j$ and $\bigcup_{j \leq n}\left(s_{j}\right)$ $=S\}$. Strictly speaking, elements of $\pi$ are subsets of $S$. We take this approach rather than supposing $S$ is finite, for example, rather than assuming $S=\left\{s_{1}, \ldots, s_{n}\right\}$. Then our analysis allows for elaborations of a given preference relation in a larger domain of acts defined over (finite) refinements of the partition $\pi$. Having made this point, we allow ourselves the familiar convention of equating the set state $s_{j}$ with its elements. That is, for notational convenience, often we shall use $s_{j}$ when we intend "members of $s_{j}$.

DeFinition 2. A simple (or discrete) horse lottery is a function from states to simple (or to discrete) lotteries. Denote a horse lottery by $H$ and denote the space of (discrete) horse lotteries on the reward set $\mathbf{R}$ by $\mathbf{H}_{\mathbf{R}}$.

In the tradition where acts are functions from states to outcomes, a horse lottery is an act with a lottery outcome. For example, the act that yields a $50-50$ chance at $\$ 10$ and $\$ 20$ provided the Republicans win the next Presidential election, and which yields a 0.25 chance at $\$ 5$ and a 0.75 chance at $\$ 10$ if the Republicans do not win, is a horse lottery over a binary partition with two states: Republicans win and Republicans do not win. Thus, a constant horse lottery is just a von Neumann-Morgenstern lottery, and a 
proper subset of these are the constant von Neumann-Morgenstern lotteries, that is, the acts which yield a specific reward for certain.

Next, we define the operation of convex combination of two horse lotteries, “+”, as the state-by-state mixture of their respective v.N-M lottery outcomes. Thus:

Definition 3. $x H_{1}+(1-x) H_{2}=H_{3}=\left\{x L_{1 j}+(1-x) L_{2 j}: j=1, \ldots, n\right.$; $0 \leq x \leq 1\}$.

The mixture of two lotteries is a lottery $x L_{1}+(1-x) L_{2}=L_{3}$, where $P_{3}(r)=x P_{1}(r)+(1-x) P_{2}(r)$. For the special cases where each $H$ is a "constant" act, that is, if $H_{1}$ is the lottery $L_{1}$ and $H_{2}$ is $L_{2}$, Definition 3 coincides with the von Neumann-Morgenstern operation of "+" for lotteries.

2.2. The axioms for order and independence. The von NeumannMorgenstern theory of preference over (simple) lotteries is encapsulated by three axioms:

1. The assumption that preference $\lesssim$ is a weak order relation.

2. The independence postulate (formulated below).

3. An Archimedean condition (discussed below).

These axioms may be applied to horse lotteries also. Then the three axioms guarantee that (i) preference is represented by a (cardinal) utility $V$ over acts with a property (ii) that utility distributes over convex combinations. To wit, given these three axioms:

(i) There exists a real-value $V$ defined on acts, unique up to positive linear transformations, where $V\left(H_{1}\right) \leq V\left(H_{2}\right)$ if and only if $H_{1} \precsim H_{2}$; and

(ii) $V\left[x H_{1}+(1-x) H_{2}\right]=x V\left(H_{1}\right)+(1-x) V\left(H_{2}\right)$.

DEFINITION 4. When a preference relation over acts satisfies (i) and (ii) we say it has the expected (or linear) utility property and we call $V$ an agreeing expected (or, linear) utility for $\precsim$.

Anscombe-Aumann theory requires a fourth postulate ensuring the existence of a unique decomposition of $V$ as a subjective expected, state-independent utility. That is, subject to a fourth postulate for preference, there exists a (unique) personal probability $p$ defined on states and a utility $U$ defined on lotteries (independent of states) so that:

$$
V(H)=\sum_{j=1}^{n} p\left(s_{j}\right) U\left(L_{j}\right) .
$$

[Recall the notation $H\left(s_{j}\right)=L_{j}$.] Key, here, is that $U$ is a state-independent utility, defined on lotteries independent of the state in which they occur. To be precise, let $H_{L}$ be the constant horse lottery that yields lottery $L$ in each state, $H_{L}\left(s_{j}\right)=L$. 
Definition 5. The utility $\left\{U_{j}: j=1, \ldots, n\right\}$ is state-independent when, for each lottery $L$ and pair of states $s_{j}$ and $s_{j^{\prime}}$,

$$
U_{j}(L)=U_{j^{\prime}}(L)=U(L) .
$$

[For our purposes, and following the usual practice, the condition of Definition 5 is required only for states $s_{j}$ and $s_{j^{\prime}}$ that are not "null," i.e., only when $p\left(s_{j}\right) \neq 0$ is it worth restricting $U_{j}$ in a decomposition of a linear utility $V$.] If the utility is state-independent, for convenience, we drop the subscript (for states) and abbreviate it $U$. When (iii) obtains for a state-independent utility $U, V\left(H_{L}\right)=U(L)$.

DEFINITION 6. When a preference relation over acts satisfies (i)-(iii) we say it has the subjective expected (state-independent) utility property and we say the pair $(p, U)$ agrees with $\lesssim$.

In contrast to (iii), a decomposition of $V$ by a subjective (possibly) statedependent utility allows

$$
V(H)=\sum_{j=1}^{n} p\left(s_{j}\right) U_{j}\left(L_{j}\right) .
$$

We examine such state-dependent decompositions in Section 4.1.

Next, we propose versions of the first two Anscombe-Aumann axioms to accommodate our theory of preference as a partial order. We postpone our discussion of the Archimedean axiom to Section 2.4 to allow for a timely account of "indifference" in Section 2.3.

In this paper, a partial order $\prec$ identifies a strict preference relation.

HL AxIOM 1. $\prec$ is a strict partial order. It is a transitive and irreflexive relation on $\mathbf{H}_{\mathbf{R}} \times \mathbf{H}_{\mathbf{R}}$.

Definition 7. When neither $H_{1} \prec H_{2}$ nor $H_{2} \prec H_{1}$, we say the two lotteries are incomparable by preference, which we denote as $H_{1} \sim H_{2}$. When $\sim$ is transitive-corresponding to a weak order-then the relation $\lesssim$ (standing for “ $\prec$ or $\sim$ ") identifies a weak preference relation. Hereafter, we shall mean by "preference" the strict preference relation.

HL Axiom 2 (Independence). $\forall\left(H_{1}, H_{2}\right.$ and $\left.H_{3}\right)$ and for each $0<x \leq 1$, $x H_{1}+(1-x) H_{3} \prec x H_{2}+(1-x) H_{3}$ if and only if $H_{1} \prec H_{2}$.

This version of independence may be used, also, as the second axiom in the Anscombe-Aumann theory or in the von Neumann-Morgenstern theory.

2.3. Indifference $(\approx)$. Next, we define a (transitive) relation of indifference, $\approx$, based on $\prec$, which will play a central role in our extension of $\prec$ to a weak order. [See Fishburn (1979), Exercises 9.1 and 9.4, pages 126-127, for additional discussion.] 
Definition 8 (Indifference). $H_{1} \approx H_{2}$ iff $\forall H_{3}, H_{4}(0<x \leq 1)$,

$$
x H_{1}+(1-x) H_{3} \sim H_{4} \text { iff } x H_{2}+(1-x) H_{3} \sim H_{4} .
$$

We establish several useful corollaries of the HL Axioms 1 and 2 about indifference.

Corollary 2.1. $\forall H_{1}, H_{2}$, if $H_{1} \approx H_{2}$, then $H_{1} \sim H_{2}$.

That is, when two acts are indifferent, neither is preferred to the other.

COROLLARY 2.2. $\approx$ is an equivalence relation.

Corollary 2.3. $H_{1} \approx H_{2}$ if and only if $\forall H_{3}, H_{4}$, and $0<x \leq 1$,

$$
x H_{1}+(1-x) H_{3} \prec(\succ) H_{4} \quad \text { iff } x H_{2}+(1-x) H_{3} \prec(\succ) H_{4} .
$$

CoROLlaRy 2.4 .

$$
\forall 0<x \leq 1, H, H_{1} \approx H_{2} \quad \text { iff } x H_{1}+(1-x) H \approx x H_{2}+(1-x) H .
$$

Corollaries 2.3 and 2.4 establish important substitution properties for elements of the same indifference equivalence class.

2.4. The Archimedean axiom: continuity of preference. First, define convergence for acts. Let $\left\{H_{n}\right\}$ be a denumerable sequence of horse lotteries.

Definition 9. $\left\{H_{n}\right\}$ converges to a lottery $H^{*}$, denoted by $\left\{H_{n}\right\} \Rightarrow H^{*}$, just in case the respective discrete lottery distributions $\left\{P_{j}^{n}(\cdot)\right\}$ converge (pointwise) to the lottery distribution $P_{j}^{*}(\cdot)$.

The third (Archimedean) axiom precludes infinitesimal degrees of preference. As we show in Theorem 4, it suffices for representing preferences by sets of agreeing real-valued utilities.

HL Axiom 3. Let $\left\{H_{n}\right\} \Rightarrow H$ and $\left\{M_{n}\right\} \Rightarrow M$.

(a) If $\forall n\left(H_{n} \prec M_{n}\right)$ and $(M \prec N)$, then $(H \prec N)$.

(b) If $\forall n\left(H_{n} \prec M_{n}\right)$ and $(J \prec H)$, then $(J \prec M)$.

The familiar Archimedean condition from Anscombe-Aumann theory (also from von Neumann-Morgenstern theory), denoted here as Axiom $3^{*}$, is this:

Axiom $3^{*}$. Whenever $H_{1} \prec H_{2} \prec H_{3}, \exists(0<x, y<1), y H_{1}+(1-y) H_{3} \prec$ $H_{2} \prec x H_{1}+(1-x) H_{3}$.

However, Axiom $3^{*}$ is overly restrictive for our purposes, as a simple example shows. 
EXAMPLE 2.1. Consider a set of three rewards $\mathbf{R}=\left\{r_{w}, r^{*}, r_{b}\right\}$ and a minimal, one element partition comprising the single sure state $\pi=\{s\}$. Then $\mathbf{H}_{\mathbf{R}}$ is the set of von Neumann-Morgenstern lotteries on $\mathbf{R}$. (Denote by $\mathbf{r}$ the horse lottery with constant prize $r$.) Let $\mathscr{V}=\left\{V_{x}: 0<x<1\right\}$ be a (convex) set of linear utilities, where $V_{x}\left(\mathbf{r}_{w}\right)=0, V_{x}\left(\mathbf{r}^{*}\right)=x$ and $V_{x}\left(\mathbf{r}_{b}\right)=1$. Figure 1 graphs these utilities.

Let $\prec_{\mathscr{V}}$ be the partial order on lotteries generated by this set of utilities according to the weak Pareto condition. That is, $L_{1} \prec_{\mathscr{V}} L_{2}$ iff $(\forall V \in \mathscr{V}$ ) $E_{V}\left[L_{1}\right]<E_{V}\left[L_{2}\right]$. By Theorem 4 (below), $\prec_{\mathscr{V}}$ satisfies HL Axioms 1 and 2. However, it fails Axiom 3*. Specifically, $\mathbf{r}_{w} \prec_{\mathscr{V}} \mathbf{r}^{*} \prec_{\mathscr{V}} \mathbf{r}_{b}$, but $\forall(0<y<1)$, $y \mathbf{r}_{w}+(1-y) \mathbf{r}_{b} \sim_{\mathscr{V}} \mathbf{r}^{*}$. Of course, $\prec_{\mathscr{V}}$ is represented by the (convex) set of agreeing (real-valued) utilities $\mathscr{V}$.

Next, we provide a connection between the Archimedean condition HL Axiom 3 and $\approx$-indifference.

CoROLlaRY 2.5. Let $\left\{H_{n}\right\},\left\{H_{n}^{\prime}\right\} \Rightarrow H ;\left\{M_{n}\right\},\left\{M_{n}^{\prime}\right\} \Rightarrow M$. If $\forall n\left(H_{n} \prec M_{n}\right.$ and $\left.M_{n}^{\prime} \prec H_{n}^{\prime}\right)$, then $H \approx M$.

We conclude this discussion of HL Axiom 3 by showing that the familiar Archimedean axiom, Axiom $3 *$, follows from our replacement HL Axiom 3 when preference over lotteries is a weak order.

LEMMA 2.1. If $\lesssim$ is a weak order over discrete horse lotteries meeting conditions HL Axioms 2 and 3, then $\lesssim$ satisfies Axiom 3*.

2.5. Utility for discrete lotteries. The theory of von Neumann and Morgenstern addresses preference over simple lotteries, that is, those with finite support. These constitute the subdomain of constant, simple horse lotteries. However, there are weakly ordered preferences over lotteries which satisfy the expected utility hypothesis (Definition 4) for simple lotteries, that is, which are represented by a cardinal, linear utility $V$ over the domain of simple lotteries, but which fail the expected utility hypothesis over the larger domain of discrete lotteries. [See Fishburn's (1979), Section 10, discussion;

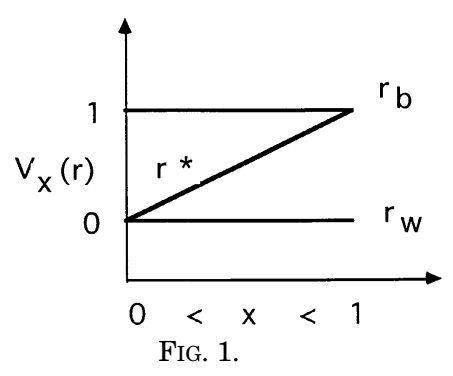


also Fishburn (1982), Section 11.3. A similar problem arises in Savage's (1954) theory, as shown by Seidenfeld and Schervish (1983). In a related matter, Aumann's $(1962,1964)$ argument about a utility for a partially ordered preference does not apply when the set of rewards is denumerable rather than finite, even though all lotteries are simple. Kannai (1963) showed that, and strengthened Aumann's Archimedean condition to remedy the problem.] We address this problem with an extended dominance condition.

Let $\mathbf{r}$ denote the simple horse lottery with constant prize $r$. Recall that $H_{L}$ denotes the constant horse lottery that yields $L$ in each state. Consider the following dominance principle:

Dominance. $\forall\left(\mathbf{r}, H_{L}\right)$ if for each $r_{n} \in \operatorname{supp}(L), \mathbf{r}_{\mathbf{n}} \prec \mathbf{r}$, then it is not the case that $\left(\mathbf{r} \prec H_{L}\right)$ [or, alternatively, if universally, $\mathbf{r} \prec \mathbf{r}_{\mathbf{n}}$, then $\operatorname{not}\left(H_{L} \prec \mathbf{r}\right)$ ].

This weak dominance condition contrasts each reward $r$ with the lottery $L$ through the (countably many) constant horse lotteries $\mathbf{r}_{\mathbf{n}}$ taken from $L$ 's support. The condition precludes a preference for $L$ over $\mathbf{r}$ if it occurs for $\mathbf{r}$ over each $\mathbf{r}_{\mathbf{n}}$.

Our first three axioms yield dominance, as the next lemma establishes.

\section{LEMma 2.2. HL Axioms 1-3 entail dominance.}

Based on Lemma 2.2, we may apply Fishburn's [(1979), page 139] Theorem 10.5 to argue that a weakly ordered preference $\lesssim$ over discrete (horse) lotteries which satisfies our HL Axioms 2 and 3 has the expected utility property. (See Remark 1.) The import for our representation theorems is given in terms of agreeing and almost agreeing utilities for a partial order:

Definition 10a. A utility $V$ agrees with a partial order $\prec$ iff $V\left[H_{1}\right]<$ $V\left[H_{2}\right]$ whenever $\left(H_{1} \prec H_{2}\right)$.

DEFINITION 10b. A utility $V$ almost agrees with a partial order $\prec$ iff $V\left[H_{1}\right] \leq V\left[H_{2}\right]$ whenever $\left(H_{1} \prec H_{2}\right)$.

Thus, when our strategy for extending a partial order $\prec$ to a weak order $\precsim$ succeeds, it induces a linear utility $V$ that agrees with $\prec$ for discrete horse lotteries, not just for simple ones.

Unfortunately, our argument for extending a partial order $\prec$ produces a set of expected utilities $\{V\}$ each of which agrees with $\prec$ for simple acts, and only almost agree with it for discrete acts. Of course, by itself the condition of "almost agreeing" is quite weak. A utility $V$ that makes all options indifferent almost agrees with every partially ordered preference. The point, however, is that we consider an almost agreeing utility for a partial order $\prec$ only in the case in which it agrees with $\prec$ on all simple acts. [This idea parallels a similar distinction between a qualitative probability (a weak order on events) and a quantitative probability that agrees or almost agrees with it. See 
Savage (1954), Section 3.3.] Through Corollary 3.2, we provide a sufficient condition for the existence of a (convex) set of utilities that agree with $\prec$ on all of $\mathbf{H}_{\mathbf{R}}$.

REMARK 1. Fishburn's Theorem 10.5 uses the traditional Archimedean axiom Axiom $3 *$. However, by Lemma 2.2 , Axiom $3 *$ follows from the assumptions that $\precsim$ is a weak order satisfying HL Axioms 2 and 3. Also, dominance is equivalent to Fishburn's [(1979, page 138] Axiom 4c, given the other three axioms and our structural assumptions about the domain of lotteries.

2.6. Bounded preferences. Next in our discussion of the axioms, we settle the question whether a partial order $\prec$ satisfying HL Axioms 1-3 admits an unbounded utility that agrees with it or agrees with it on simple lotteries. It is well known that utilities for von Neumann-Morgenstern lotteries are finite. In light of our assumption that all discrete lotteries are acts, utilities that agree with $\prec$ are bounded as well.

Corollary 2.6. Let $\prec$ satisfy HL Axioms 1 and 2. If $V$ agrees with $\prec$, it is bounded. Hence, all utilities that agree with $\prec$ are bounded.

As noted above, we sometimes construct a utility $V$ that agrees with a partial order $\prec$ for all simple lotteries but (merely) almost agrees with $\prec$ for discrete lotteries. Thus, as $V$ may fail to agree with $\prec$ on nonsimple acts, it is worthwhile to show (appealing only to simple acts) that each utility $V$ we construct is bounded. For this purpose we formalize a condition that a partially ordered preference is bounded, and establish it as a corollary of two of our axioms, HL Axioms 1 and 3. From the fact that a partial order $\prec$ is bounded, we show that a utility $V$ agreeing with it on simple acts also is bounded.

Call a countable (finite or denumerably infinite) sequence of lotteries $\left\{H_{n}\right.$ : $n=1, \ldots\}$ an increasing (decreasing) chain if $H_{i} \prec H_{j}\left(H_{j} \prec H_{i}\right)$ whenever $i<j$. The following concepts deal with chains of strict preference. $\left\{H_{n}\right\}$,

DEFINITION 11a. Say $\prec$ is bounded above if, for each increasing chain

$$
\lim _{n \rightarrow \infty} \sup \left\{x:\left(H_{2} \prec x H_{1}+(1-x) H_{n}\right)\right\}<1
$$
$\left\{H_{n}\right\}$,

Definition 11b. Say $\prec$ is bounded below if, for each decreasing chain

$$
\lim _{n \rightarrow \infty} \sup \left\{x:\left(x H_{1}+(1-x) H_{n} \prec H_{2}\right)\right\}<1 .
$$

Definition 11c. Call $\prec$ bounded if it is bounded both above and below. 
LEMMA 2.3. If $\prec$ satisfies HL Axioms 1 and 3 , then $\prec$ is bounded, that is, all $\prec$-chains are bounded.

Also, Lemma 2.3 yields the following claim about utilities for rewards:

COROLlary 2.7. Let $W$ be a (real-valued) utility and assume that, in the domain of simple lotteries, its strict order $\prec_{W}$ satisfies HL Axioms 1 and 3. Then $\sup _{\mathbf{R}}|W(r)|<\infty$.

Recall that a linear utility $V$ is defined only up to a positive linear transformation. We use the facts reported by Corollaries 2.6 and 2.7 to standardize the units $(0$ and 1$)$ for each $V$ in a set of agreeing utilities $\mathscr{V}$ (agreeing with $\prec$ on simple acts, at least).

Definition 11d. A set $\mathscr{V}=\{V\}$ of utilities is bounded if, for some standardization of its elements,

$$
\sup _{\mathscr{V}, \mathbf{H}_{\mathbf{R}}}|V(H)|<\infty .
$$

The problem we face is this. Though each $V \in \mathscr{V}$ is bounded, there exist what are for our purposes undesirable standardizations of the $V$ 's which fail to satisfy Definition 11d. For an illustration, recall Example 2.1. There, the domain of (simple) lotteries $\mathbf{H}_{\mathbf{R}}$ is generated by three rewards $\mathbf{R}=\left\{r_{w}, r^{*}, r_{b}\right\}$ using a partition of one (sure) state. That is, Example 2.1 is about preferences over von Neumann-Morgenstern lotteries. A partially ordered preference $\prec_{\mathscr{V}}$ over $\mathbf{H}_{\mathbf{R}}$ arises (by the Pareto rule) from the convex set of utilities $\mathscr{V}=$ $\left\{V_{x}: 0<x<1\right\}$, where $V_{x}\left(\mathbf{r}_{w}\right)=0, V_{x}\left(\mathbf{r}^{*}\right)=x$ and $V_{x}\left(\mathbf{r}_{b}\right)=1$. That is, $H_{L 1} \prec_{\mathscr{V}}$ $H_{L 2}$ iff $V\left(H_{L 1}\right)<V\left(H_{L 2}\right)$ for each $V \in \mathscr{V}$.

Obviously, the two constant acts (the rewards) $\mathbf{r}_{\mathbf{w}}$ and $\mathbf{r}_{\mathbf{b}}$ bound the partial order $\prec_{\mathscr{V}}$, that is, for each act $H_{L}$ different from $\mathbf{r}_{\mathbf{w}}$ and $\mathbf{r}_{\mathbf{b}}, \mathbf{r}_{\mathbf{w}} \prec_{\mathscr{V}} H \prec_{\mathscr{V}} \mathbf{r}_{\mathbf{b}}$. Moreover, in this standardization of $\mathscr{V}, \sup _{\mathscr{V}, \mathbf{H}_{\mathbf{R}}}|V(H)|=1$. Hence, it is a bounded set of utilities. However, we may standardize the elements of $\mathscr{V}$ so that it fails the condition in question. Rewrite each $V_{X}$, instead, so that $V_{X}\left(\mathbf{r}_{w}\right)=0, V_{X}\left(\mathbf{r}^{*}\right)=1$ and $V_{X}\left(\mathbf{r}_{b}\right)=1 / X$. Then $\lim _{X \rightarrow 0} V_{X}\left(\mathbf{r}_{b}\right)=\infty$.

To ensure a simple standardization which establishes our $\mathscr{V} \mathrm{s}$ are, indeed, bounded sets of utilities, we verify that (without loss of generality) we may introduce two rewards $W$ and $B$ (analogous to $r_{w}$ and $r_{b}$ in Example 2.1) that serve to bound the preferences for all other acts: Theorem 2 . Then, the sets $\mathscr{V}$ are bounded sets of utilities since we standardize all $V \in \mathscr{V}$ with $V(\mathbf{W})=0$ and $V(\mathbf{B})=1$.

2.7. Standardizing <-preferences with "best" and "worst" acts. In this section we show how to extend the domain of a partially ordered preference by bounding it with "worst" and "best" acts. First, however, we review two concepts of "null" events. 
Definition 12. An event $e$ is the set of states in a subset $T$ of $\pi:(\forall e) \exists$ $(T \subset \pi), e=\cup_{s \in T}[s]$.

Definition 13. Call $H_{1}$ and $H_{2}$ a pair of e-called-off acts when $H_{1}(s)=$ $\mathrm{H}_{2}(s)$ if $s \notin e$.

Distinguish two senses of "null" events.

Definition 14a. An event $e$ is potentially null iff for each pair of $e$-calledoff acts $H_{1}$ and $H_{2}, H_{1} \sim H_{2}$.

DEFINITION 14b. Event $e$ is essentially null iff for each pair of $e$-called-off acts $H_{1}$ and $H_{2}, H_{1} \approx H_{2}$.

It is evident that when event $e$ is essentially null, so too is each state that comprises it. Denote by $\mathbf{n}$ the union of the essentially null states. It follows (as is proven next) that the union of essentially null states is an essentially null event. Hence, $\mathbf{n}$ is the maximal essentially null event.

Corollary 2.8. Let $N \subset \pi$ be the subset of all essentially null states $N=\left\{s_{j_{1}}, \ldots, s_{j_{k}}\right\}$, with $\mathbf{n}=\cup_{s \in N}(s)$. Then $\mathbf{n}$ is essentially null.

THEOREM 2. Assume $\prec$ is a partially ordered preference (satisfying $H L$ Axioms 1-3) over a set of discrete horse lotteries $\mathbf{H}_{\mathbf{R}}$, defined on the partition $\pi^{n}=\left\{s_{j}: j=1, \ldots, n\right\}$. Let $\mathbf{R}^{\prime}=\mathbf{R} \cup\{\mathbf{W}, \mathbf{B}\}$, where neither $W$ nor $B$ is an element of $\mathbf{R}$. Then we may extend $\prec$ to a partially ordered preference $\prec$ ' over $\mathbf{H}_{\mathbf{R}}$, so that:

1. $\prec^{\prime} / \mathbf{H}_{\mathbf{R}}=\prec$. That is, $\prec^{\prime}$ restricted to $\mathbf{H}_{\mathbf{R}}$ is just $\prec$.

2. $\forall\left(H \in \mathbf{H}_{\mathbf{R}}\right), \mathbf{W} \prec^{\prime} H \prec^{\prime} \mathbf{B}$.

3. $\prec^{\prime}$ satisfies HL Axioms 1-3.

Since $\mathbf{n}=S$ iff $\prec$ is trivial, that is, iff $\forall\left(H_{1}, H_{2}\right), H_{1} \approx H_{2}$ [also, iff $\forall$ $\left(H_{1}, H_{2}\right), H_{1} \sim H_{2}$ ], without loss of generality, by Theorem 2 , assume preference is not trivial by including rewards $W$ and $B$. (This proposition, warranted by Theorem 2, is the counterpart in our theory to Savage's P5.)

\section{Extending strict partial orders: the inductive argument.}

3.1. An overview. Let $\mathbf{R}=\left\{r_{1}, r_{2}, \ldots\right\}$ be a countable (finite or denumerable) set of rewards and let $\prec$ be a preference over $\mathbf{H}_{\mathbf{R}}$ satisfying HL Axioms 1-3. Based on Theorem 2, without loss of generality, assume the existence of two distinguished rewards not in $\mathbf{R}$ : reward $W$, where $\mathbf{W}$ is the worst act, and reward $B$, where $\mathbf{B}$ is the best act. Acts $\mathbf{W}$ and $\mathbf{B}$ are to serve as the common 0 and 1 in a (convex) set $\mathscr{V}$ of bounded utility functions $V$ that agree with $\prec$. Hence for all $H \in \mathbf{H}_{\mathbf{R}}, \mathbf{W} \prec H \prec \mathbf{B}$.

Let us highlight the major results in this section of our essay. 
Our strategy is to use a transfinite induction to extend the preference $\prec$ (a partial order) to a weak order $\precsim$ over simple horse lotteries in $\mathbf{H}_{\mathbf{R}}$. Let $\prec$ $\left(=\prec_{0}\right)$ serve as the basis for the induction. The induction at the $i$ th stage extension of $\prec, \prec_{i}$, obtains by assigning a utility $v_{i}$ to act $\tilde{H}_{i}, V\left(\tilde{H}_{i}\right)=v_{i}$, so that $\tilde{H}_{i} \approx_{i} v_{i} \mathbf{B}+\left(1-v_{i}\right) \mathbf{W}$. The quantity $v_{i}$ is chosen (in accord with Definitions 20 and 25 in the Appendix) from a (convex) set of target utilities for $\tilde{H}_{i}, \mathscr{T}_{i}\left(\tilde{H}_{i}\right)$. The sequence $\left\{\tilde{H}_{i}\right\}$ is chosen (see Definition 26 in the Appendix) so that the limit stage $\prec_{\omega}$ is a weak order over $\mathbf{H}_{\mathbf{R}}$. We use $\mathbf{W}$ and $\mathbf{B}$ as the 0 and 1 of our utility, as follows.

Assume $\left\{H_{n}\right\} \Rightarrow H$ and $H_{n} \in \mathbf{H}_{\mathbf{R}}$. The general target sets $\mathscr{T}_{i}(H)$ are defined through endpoints that bound the candidate utilities:

Definition 17. Let $v_{i}^{*}(H)$ be the liminf of the quantities $x_{n}$ for which $H_{n} \prec_{i-1} x_{n} \mathbf{B}+\left(1-x_{n}\right) \mathbf{W}$.

Definition 18. Let $v_{i *}(H)$ be the lim sup of the quantities $x_{n}$ for which $x_{n} \mathbf{B}+\left(1-x_{n}\right) \mathbf{W} \prec_{i-1} H_{n}$.

[The "utility" bounds $v_{*}(H)$ and $v^{*}(H)$ do not depend upon which sequence $\left\{H_{n}\right\} \Rightarrow H$ is used, as explained in the Appendix.] Next, define the (closed) target set of utilities for an act $H \in \mathbf{H}_{\mathbf{R}}$ :

Definition 19. $\mathscr{T}(H)=\left\{v: v_{*}(H) \leq v \leq v^{*}(H)\right\}$.

We report two key properties of $\mathscr{T}(H)$ with the following lemma.

LEMma 3.1. Assume $\prec$ satisfies the three axioms. Then:

(i) $v_{*}(H) \leq v^{*}(H)$; and

(ii) $v_{*}(H)=v^{*}(H)=v_{H}$ iff $H \approx v_{H} B+\left(1-v_{H}\right) W$.

Our plan succeeds because $\prec_{i}$ extends $\prec_{i-1}$, it satisfies the three HL axioms and it preserves the $\approx_{i-1}$-indifference relations. Each weak order $\Im_{V}=\lessgtr_{\omega}$ (corresponding to the limit stage relation " $\prec_{\omega}$ or $\approx_{\omega}$ ") is defined by inequalities in expected $V$-utility, based on the utilities $v_{i}$ for each act $\tilde{H}_{i}$ in a (finite or) denumerable class $\mathscr{H} \subset \mathbf{H}_{\mathbf{R}}$. (As explained below, $\mathscr{H}$ is finite or denumerable depending upon whether $\mathbf{R}$ is.) We choose $\mathscr{H}$ to form a basis for $\precsim_{V}$, that is, each $H \in \mathbf{H}_{\mathbf{R}}$ is a limit point of simple acts and each simple act has its utility fixed by some finite stage of the transfinite induction. Then, $\nwarrow_{V}$ extends $\prec$ on simple acts in $\mathbf{H}_{\mathbf{R}}$. Also, the utility $V$ almost agrees with $\prec$ over the discrete lotteries $\mathbf{H}_{\mathbf{R}}$. That is, if $H_{1} \prec H_{2}$, then $H_{1} \precsim_{V} H_{2}$. In Corollary 3.1, we provide sufficient conditions under which $\precsim_{V}$ extends $\prec$ for all the acts in $\mathbf{H}_{\mathbf{R}}$. (See Remark 2.)

We show in Theorem 4 that each set $\mathscr{Z}$ of (bounded, standardized) real-valued utility functions over $\mathbf{R}$ induces a partial order, $\prec_{\mathscr{Z}}$, according to the Pareto preference relation, and $\prec_{\mathscr{Z}}$ satisfies our axioms. Of course, each 
utility $Z \in \mathscr{Z}$ agrees with $\prec_{\mathscr{Z}}$. That is, $\mathscr{Z}$ is a subset of the set of all utilities agreeing with $\prec_{\mathscr{Z}}$. However [Seidenfeld, Schervish and Kadane (1990), E.3], distinct convex sets of bounded utilities may induce the same strict partial order. Thus, our representation of the partial order $\prec$ is in terms of the largest convex set of agreeing linear utilities - the union of all sets of utilities where each set induces $\prec$ according to the Pareto condition.

Assume $\prec$ satisfies our axioms and let $\mathscr{Z}^{\mathscr{S}}$ be the nonempty (convex) set of bounded utilities that agree with $\prec$ for simple acts. That is, $\mathscr{Z}^{\mathscr{S}}$ is the set of all bounded utilities with the property that, for simple acts $H_{1}$ and $H_{2}$, $H_{1} \prec H_{2}$ only if for each utility $Z$ in $\mathscr{Z}^{\mathscr{S}}$, the expected $Z$-utility of $H_{2}$ is greater than that of $H_{1}$. Let $\mathscr{V}$ be the nonempty (convex) set of utilities created for $\prec$ by (our method of induction in) Theorem 3 . Theorem 5 asserts $\phi \neq \mathscr{V}=\mathscr{Z}^{\mathscr{S}}$. Last, when the conditions of Corollary 3.1 apply, then (Corollary 3.2) $\mathscr{V}$ is the nonempty set of all utilities that agree with $\prec$.

REMARK 2. If the Archimedean axiom is ignored and only simple lotteries are considered, the induction for extending $\prec$ to a weak order (in fact, to a total order) $\lesssim$ over $\mathbf{H}_{\mathbf{R}}$ is elementary and applies without a cardinality restriction on the reward set $\mathbf{R}$ and without need of the special acts $\mathbf{W}$ and $\mathbf{B}$. See the Appendix to Schervish and Kadane (1990). There, we show the following: Let $\kappa$ be the cardinality of $\mathbf{R}$. Using Hausner's (1954) result, the order $\precsim\left(=\prec_{K}\right)$ is a lexicographic expected utility.

\subsection{The central theorem.}

TheOREm 3. Let $\prec$ be a nontrivial partial order over $\mathbf{H}_{\mathbf{R}}$ satisfying $\mathrm{HL}$ Axioms 1-3. Then:

(i) For simple lotteries in $\mathbf{H}_{\mathbf{R}}, \prec$ can be extended to a weak order $\lessgtr_{\omega}=\lessgtr$ satisfying HL Axioms 2 and 3. That is, $\lesssim$ is uniquely represented by a (bounded) real-valued utility $V$ over $\mathbf{R}$ which agrees with $\prec$ for simple acts. In symbols, $\forall$ (simple $\left.H_{1}, H_{2} \in \mathbf{H}_{\mathbf{R}}\right)$, if $\left(H_{1} \prec H_{2}\right)$, then $E_{V}\left[H_{1}\right]<$ $E_{V}\left[H_{2}\right]$, and if $\left(H_{1} \approx H_{2}\right)$, then $E_{V}\left[H_{1}\right]=E_{V}\left[H_{2}\right]$.

(ii) V almost agrees with $\prec . \forall\left(H_{1}, H_{2} \in \mathbf{H}_{\mathbf{R}}\right)$, if $\left(H_{1} \prec H_{2}\right)$, then $E_{V}\left[H_{1}\right]$ $\leq E_{V}\left[H_{2}\right]$.

It is instructive to illustrate how $\nwarrow_{\omega}$ may fail to agree with $\prec$ for some nonsimple lotteries in $\mathbf{H}_{\mathbf{R}}$. The example motivates a condition on $\prec$ which proves sufficient for $\prec_{\omega}$ to extend $\prec$.

EXAMPLE 3.1. Let $\mathscr{W}=\left\{W_{j}: j=1, \ldots\right\}$ be a countable set of utilities on $\mathbf{R}=\left\{r_{i}: i=1, \ldots\right\}$ with the two properties that $W_{j}\left(\mathbf{r}_{m}\right)=0.25$ if $m \neq 2 j$, while $W_{j}\left(\mathbf{r}_{2 j}\right)=0.5$. According to Theorem 4 (below), under the (weak) Pareto rule, $\mathscr{W}$ induces a partial order $\prec_{\mathscr{W}}$ which satisfies our three horse lottery axioms. Define the constant, nonsimple acts $H_{a}$ and $H_{b}$ by $H_{a}=\left\{P\left(r_{i}\right)=\right.$ 
$1 / 2^{m}$ if $i=2 m-1, P\left(r_{i}\right)=0$ otherwise $\}$ and $H_{b}=\left\{P\left(r_{i}\right)=1 / 2^{m}\right.$ if $i=2 m$, $P\left(r_{i}\right)=0$ otherwise $\}$. Then, evidently $\left(H_{a} \prec_{\mathscr{V}} H_{b}\right)$. However, at the $k$ th stage $\prec_{k}$ in the extension of $\prec_{\mathscr{W}}$, we may arrange our choices of utilities for rewards so that $V\left(r_{k}\right)=0.25(k=1, \ldots)$. However, then $\precsim_{\omega}$ does not extend $\prec_{\mathscr{W}}$ as $H_{a} \approx_{\omega} H_{b}$.

Definition 28. Given two subsets of $\prec$-preferences $\mathscr{Q}$ and $\mathscr{R}$, say that $\mathscr{Q}$ is a basis for $\mathscr{R}$ if every preference, $\left(H_{1}<H_{2}\right) \in \mathscr{R}$, is a consequence (under HL Axioms 1-3) of preferences in $\mathscr{Q}$.

COROLlaRY 3.1. If there exists a countable basis $\mathscr{B}$ for $\prec$, then there exists a (bounded) real-valued utility $V$ and corresponding weak order $\lessgtr$ that agrees with $\prec$ on all of $\mathbf{H}_{\mathbf{R}}$. (Thus, a sufficient condition for the existence of an agreeing $\precsim$ is that $\prec$ is a separable partial order.)

Next, we show that our axioms are not overly restrictive for representing a partial order by a (convex) set of agreeing utilities. We investigate relationships between a partial order $\prec_{\mathscr{Z}}$ (formed by the Pareto rule with a set $\mathscr{Z}$ of utilities) and the set $\mathscr{V}$ of utilities created by induction on $\prec_{\mathscr{Z}}$. Let $\mathscr{Z}$ be a set of bounded utilities on $\mathbf{R}$, standardized so that for $Z \in \mathscr{Z}$ and $H \in \mathbf{H}_{\mathbf{R}}$, $0=Z(\mathbf{W})<Z(H)<Z(\mathbf{B})=1$. Define the relation $\prec_{\mathscr{Z}}$ on $\mathbf{H}_{\mathbf{R}}$ by the Pareto condition:

Definition 29. $\left(H_{1} \prec_{\mathscr{Z}} H_{2}\right)$ iff $\forall Z \in \mathscr{Z}, Z\left(H_{1}\right)<Z\left(H_{2}\right)$.

THEOREM 4. $\prec_{\mathscr{Z}}$ satisfies HL Axioms 1-3.

Next, let $\mathscr{V}$ be the set of utilities that can be generated from the partial order $\prec$ according to our induction. Let $\mathscr{Z}^{\mathscr{S}}$ be the set of all bounded utilities $Z$ that agree with $\prec$ on simple lotteries.

THEOREM 5. $\phi \neq \mathscr{V}=\mathscr{Z}^{\mathscr{S}}$.

Last, assume $\prec$ satisfies our axioms, let $\mathscr{Z}$ be the set of all utilities that agree with $\prec$ and let $\mathscr{V}$ be the set of utilities created by (our induction in) Theorem 3. We state three immediate corollaries of Theorem 5:

COROLLARY 3.2. When $\prec$ satisfies the separability condition of Corollary 3.1 , then $\phi \neq \mathscr{V}=\mathscr{Z}$.

Corollary 3.3. The set $\mathscr{V}$ does not depend upon the ordering of $\mathscr{H}$.

Corollary 3.4. The set $\mathscr{V}$ is convex. 


\section{A representation of $\prec$ in terms of probabilities and state- dependent utilities.}

4.1. The underdetermination of personal probability by HL Axioms 1-3. Let $\mathscr{V}$ be the set of utilities $V$, each of which (by Theorem 5) corresponds to a limit stage $\nwarrow_{V}$ in our inductive extensions of the partial order $\prec$. According to Theorem $5, \mathscr{V}$ is the set of all and only utilities that agree with $\prec$ on simple acts. According to Corollary 3.2, when $\prec$ is separable, $\mathscr{V}$ is the set of utilities that agree with $\prec$. We examine decompositions of $V \in \mathscr{V}$ as a subjective expected (state-dependent) utility.

Let $\precsim$ be a weak order over the discrete horse lotteries $\mathbf{H}_{\mathbf{R}}$. Let $p(\cdot)$ be a (personal) probability defined on states in $\pi$, with $P(\mathbf{n})=0$ for the set of $\precsim$-null states $\mathbf{n}$. Finally, let $U_{j}(\cdot)$ be a (possibly) state-dependent utility on the discrete v.N-M lotteries $\mathbf{L}_{\mathbf{R}}$, defined for the $\precsim$-nonnull states $s_{j}$. That is, for each $\precsim$-nonnull state, $s_{j} \notin \mathbf{n}, U_{j}$ is a v.N-M utility. (For completeness, we may take $U_{j}$ to be a constant function when $s_{j} \in \mathbf{n}$.)

DeFINITION 30. Say that $\precsim$ represented as a subjective expected (statedependent) utility by the pair $\left(p,\left\{U_{j}: j=1, \ldots, n\right\}\right)$, whenever

$$
H_{1} \precsim H_{2} \text { iff } \sum_{j} p\left(s_{j}\right) U_{j}\left(L_{1 j}\right) \leq \sum_{j} p\left(s_{j}\right) U_{j}\left(L_{2 j}\right) .
$$

For convenience, abbreviate the probability/(state-dependent) utility pairs as $\left(p, U_{j}\right)$.

We rely on a result due to Fishburn [(1979), Theorem 13.1] to show that each $\Im_{V}, V \in \mathscr{V}$, bears the subjective expected utility property for a large class of $\left(p, U_{j}\right)$ pairs. In fact, for each such $\preccurlyeq_{V}$, the $\left(p, U_{j}\right)$ pairs range over all mutually absolutely continuous probabilities defined on the $\Im_{V}$-nonnull states. Specifically:

LEMMA 4.1. Let $\lesssim$ be a (nontrivial) weak order on $\mathbf{H}_{\mathbf{R}}$ satisfying $\mathrm{HL}$ Axioms 2 and 3. For each probability $p(\cdot)$ with support the (nonempty) set of $\precsim$-nonnull states, there is a ( possibly) state-dependent utility $U_{j}(\cdot)$ on discrete lotteries for which $\lesssim$ has property (4.1) under $\left(p, U_{j}\right)$. lary:

Putting Theorem 5 and Lemma 4.1 together, we have the following corol-

Corollary 4.1. There exists a set of pairs $\left\{\left(p(\cdot), U_{j}(\cdot)\right)\right\}$, with $p$ a personal probability defined on the set of $\lessgtr_{V}$-nonnull states in $p$ and $U_{j}$ a state dependent utility over the discrete lotteries, where $\forall\left(H \in \mathbf{H}_{\mathbf{R}}\right), V(H)=$ $\sum_{j} p\left(s_{j}\right) \times U_{j}\left(L_{j}\right)$.

[Being linear utilities, the $U_{j}$ have the expected utility property for lotteries. That is, $U_{j}\left(x L_{1}+(1-x) L_{2}\right)=x U_{j}\left(L_{1}\right)+(1-x) U_{j} L_{2}$. Moreover, for each $V \in \mathscr{V}$, the set of personal probabilities $\left\{p: \exists U_{j}\right.$ with $\precsim_{V}$ represented by $\left.\left(p, U_{j}\right)\right]$ is closed under the relation of mutual absolute continuity.] 
4.2. State-independent utilities and a counterexample. Anscombe and Aumann's theory of horse lotteries introduces a fourth axiom which suffices for a unique expected utility representation of a weak order $\lesssim$ by a pair $(p, U)$, with $p$ a personal probability over states and $U$ a state-independent utility over rewards.

Recall Definition 5, when $U$ is state-independent, a lottery $L$ has the same utility independent of the (nonnull) state $s_{j}$. Hence [as in Savage's (1954) theory], $U$ assigns a constant utility across (nonnull) states to each "constant" act. In Anscombe and Aumann's theory, then (4.1) is strengthened to read:

$$
H_{1} \precsim H_{2} \quad \text { iff } \quad \sum_{j} p\left(s_{j}\right) U\left(L_{1 j}\right) \leq \sum_{j} p\left(s_{j}\right) U\left(L_{2 j}\right)
$$

and each $\lesssim$ is so represented by a unique $(p, U)$ pair.

The existence of a state-independent utility for $\precsim$ is assured through a contrast between (unconditional) preferences over constant horse lotteries and preferences over $s_{j}$-called-off horse lotteries: pairs of acts that differ only in one state. Specifically, let $H_{L_{i}}(i=1,2)$ be two constant horse lotteries that award, respectively, the v.N-M lottery $L_{i}$ in all states. Let $H_{i}(i=1,2)$ be two $s_{j}$-called-off horse lotteries with $H_{i}\left(s_{j}\right)=L_{i}$ [and $H_{1}(s)=H_{2}(s)$ for $s \neq s_{j}$ ]. The Anscombe-Aumann (AA) axiom for state-independent utility reads:

AA Axiom 4. Provided $s_{j} \notin \mathbf{n}$, for each such quadruple of acts, $H_{L_{1}} \precsim H_{L_{2}}$ iff $H_{1} \preccurlyeq H_{2}$.

(Recall, their Axiom 1 stipulates that preferences are weakly ordered, $\lesssim$; hence, in their theory there is no difference between "potentially null" and "essentially null" states.)

This axiom requires that $\precsim$-preferences over "constant" acts (such as the $H_{L_{i}}$ ) are reproduced by called-off choices (the $H_{i}$ ) given each nonnull $s_{j}$. The unconditional preference for v.N-M lotteries is their conditional (that is, called-off) preference, given a nonnull state. (We discuss conditional partially ordered preferences in Section 5.)

It is significant to understand that AA Axiom 4, though sufficient to create state-independent utilities when preference satisfies the usual ordering, independence and Archimedean conditions, does not preclude alternative expected utility representations by state-dependent utilities. Lemma 4.1 continues to apply, even in the presence of the extra axiom for state-independent utilities. Weak orderings that satisfy the independence, Archimedean and state-independent utility axioms admit a continuum of different probability/utility representations, each in accord with (4.1).

What the Anscombe-Aumann fourth axiom achieves, however, is to guarantee that precisely one probability/utility pair, among the set of all pairs $\left\{\left(p, U_{j}\right)\right\}$ indicated by Lemma 4.1, satisfies the more restrictive condition, (4.2). In Anscombe and Aumann's theory, as in Savage's theory, this probability/utility pair $(p, U)$ is given priority over the others. That is, these theories 
select the one (and only one) expected state-independent utility representation of preference, in accordance with (4.2) and, thereby, fix a personal probability uniquely from $\precsim$-preferences.

We are not satisfied with a conventional resolution of the representation problem indicated by Lemma 4.1. If state-dependent utilities are plausible candidates for an agent's values, and we think sometimes they are, then the measurement question remains open despite the fourth axiom. What justification is there for a convention which gives priority to state-independent values? In two essays [Schervish, Seidenfeld and Kadane (1990, 1991)], we examine the case of weakly ordered preferences without the extra axiom for "state-independent" utility. Here, instead, we adopt the strategy of imposing a modified Axiom 4 and asking which probability/state-independent utility pairs agree with the partial order $\prec$. Unlike the Anscombe-Aumann or Savage theories, ours does not assert that these pairs of probability/(stateindependent) utility functions identify the agent's degrees of beliefs and values.

We adapt Anscombe and Aumann's final axiom to our construction by restricting it to states which are not potentially null. This produces the following axiom:

HL AXIOM 4. If $s_{k}$ is not $\prec$-potentially null, then for each quadruple of acts $H_{L_{i}}, H_{i}(i=1,2)$ as described above, $H_{L_{1}} \prec H_{L_{2}}$ iff $H_{1} \prec H_{2}$.

Suppose, $\prec$ is a preference on horse lotteries subject to HL Axioms 1-4. Surprisingly, there may not exist a probability and state-independent utility agreeing with $\prec$ [according to (4.2)], even for simple acts. Moreover, the problem has nothing to do with existence of potentially null states. That is, even if no state is potentially null, the fourth axiom (HL Axiom 4) is insufficient for the existence of a probability/state-independent utility pair agreeing with $\prec$.

EXAMPLE 4.1. Let $\mathbf{R}=\left\{r_{*}, r, r^{*}\right\}$ be three rewards and consider the set $\mathbf{H}_{\mathbf{R}}$ of horse lotteries defined on the binary partition $\left\{s_{1}, s_{2}\right\}$. Next, consider two probability /utility pairs $\left(p^{i}, U^{i}\right)(i=1,2)$, where $U^{i}\left(r_{*}\right)=0, U^{i}\left(r^{*}\right)=1$, $U^{1}(r)=0.1$ and $U^{2}(r)=0.4$; also, $p^{1}\left(s_{1}\right)=0.1$ and $p^{2}\left(s_{1}\right)=0.3$. Define $H_{1} \prec H_{2}$ iff $p^{i}\left(s_{1}\right) U^{i}\left(L_{1,1}\right)+p^{i}\left(s_{2}\right) U^{i}\left(L_{1,2}\right)<p^{i}\left(s_{1}\right) U^{i}\left(L_{2,1}\right)+$ $p^{i}\left(s_{2}\right) U^{i}\left(L_{2,2}\right)(i=1,2)$. Then, by Theorem 4, and we claim it satisfies HL Axiom 4 as well. Moreover neither state is potentially null under $\prec$.

The proof that $\prec$ satisfies HL Axiom 4 is straightforward. We observe the following (expected utility) bounds on $\prec$-preferences for the constant horse lottery r. $\quad(\forall 0.1>\varepsilon>0),(0.9+\varepsilon) \mathbf{r}_{*}+(0.1-\varepsilon) \mathbf{r}^{*} \prec \mathbf{r} \prec(0.6-\varepsilon) \mathbf{r}_{*}+$ $(0.4+\varepsilon) \mathbf{r}^{*}$. However, the utilities $U^{i}$ are state-independent and neither state is null for either $p^{i}(i=1,2)$. That is, using conditional preference (see Definition 34, $(\forall 0.1>\varepsilon>0)(0.9+\varepsilon) \mathbf{r}_{*}+(0.1-\varepsilon) \mathbf{r}^{*} \prec \mathbf{s}_{\mathbf{j}}, \quad \mathbf{r} \prec \mathbf{s}_{\mathbf{j}}(0.6-$ 
$\varepsilon) \mathbf{r}_{*}+(0.4+\varepsilon) \mathbf{r}^{*}(j=1,2)$. The utility bounds for $\mathbf{r}$ reproduce in both families of $s_{j}$-called-off acts. Hence, $\prec$ satisfies HL Axiom 4.

According to Theorem 1, the two pairs $\left(p^{i}, U^{i}\right)$ are the sole state-independent expected utilities agreeing with $\prec$ [according to (4.2)]. Next, we assert that $\prec$ may be extended to a strict partial order $\prec$ ", also satisfying HL Axioms 2-4, but where $\prec "$ narrows the expected utility bounds for $r$, as follows: $0.9 r_{*}+0.1 r^{*} \prec " \mathbf{r} \prec " 0.6 r_{*}+0.4 r^{*}$.

We outline a general result for extending $\prec$ by forcing a new strict preference $H_{1} \prec^{\prime} H_{2}$, when $H_{1} \sim H_{2}$. This contrasts with the extension created through Definition 20, which, instead, forces a new indifference relation.

Suppose $H_{1}$ and $H_{2}$ are elements of $\mathbf{H}_{\mathbf{R}}$ that satisfy (1) $H_{1} \sim H_{2}$ and (2) there do not exist two sequences $\left\{H_{i, n}\right\} \Rightarrow H_{i}(i=1,2)$, where $\forall(n=1, \ldots)$, $H_{2, n} \prec H_{1, n}$. Create an extension $\prec^{\prime}$ of $\prec$ as follows:

Definition $\left(\prec^{\prime}\right) . \quad \forall\left(H_{a}, H_{b} \in \mathbf{H}_{\mathbf{R}}\right), H_{a} \prec^{\prime} H_{b}$ if and only if either:

or

(i) $H_{a} \prec H_{b}$ (so $\prec^{\prime}$ extends $\prec$ )

(ii) $\exists\left\{H_{a, n}\right\} \Rightarrow H_{a}$ and $\exists\left\{H_{b, n}\right\} \Rightarrow H_{b}$ and $\exists\left\{x_{n}\right\}$ with $\lim _{n \rightarrow \infty}\left\{x_{n}\right\} \neq 1$,

$$
x_{n} H_{a, n}+\left(1-x_{n}\right) H_{2} \prec x_{n} H_{b, n}+\left(1-x_{n}\right) H_{1} \text {. }
$$

Claim. $\quad \prec^{\prime}$ satisfies HL Axioms 1-3, provided $\prec$ does. Also, $H_{1} \prec ' H_{2}$.

We omit the proof which follows along similar lines for the demonstration of Lemma 3.3. Regarding HL Axiom 4, it suffices that $\prec^{\prime}$ is formed by extending $\prec$ using a target set endpoint, for example, let $H_{1}=v_{*} B+(1-$ $\left.v_{*}\right) W$, where $\mathscr{T}\left(H_{2}\right)=\left[v_{*}, v^{*}\right]$ and this interval has interior, that is, $v_{*}<v^{*}$. Then $\prec$ ' satisfies HL Axiom 4 too.

Last, for Example 4.1, apply the claim, twice over, first to force $0.9 \mathbf{r}_{*}+$ $0.1 \mathbf{r}^{*} \prec^{\prime} \mathbf{r}$, then to force $\mathbf{r} \prec^{\prime \prime} 0.6 \mathbf{r}_{*}+0.4 \mathbf{r}^{*}$.

Consider the convex sets $\mathscr{V}$ and $\mathscr{V}^{\prime \prime}$ of agreeing utilities for $\prec$ and $\prec$ " provided by Corollary 3.2. (These utilities agree since $\mathbf{R}$ is finite.) Because $\prec$ " extends $\prec$, then $\mathscr{V}^{\prime \prime} \subset \mathscr{V}$. A fortiori, each agreeing expected stateindependent utility model for $\prec "$ also is one for $\prec$. However, by Theorem 1 , there does not exist an agreeing expected state-independent utility model for $V^{\prime \prime} \in \mathscr{V}^{\prime \prime}$, since $\mathscr{V}^{\prime \prime}$ excludes all (that is, both) expected state-independent utility models for $\prec$. Nonetheless, $\prec "$ satisfies HL Axioms 1-4. This ends our discussion of Example 4.1.

4.3. Representation of $\prec$ in terms of (nearly) state-independent utilities. The four axioms HL Axioms 1-4 are insufficient for the existence of an agreeing state-independent utility. However, with the addition of a fifth axiom to regulate state-dependence for potentially null states, the resulting theory is sufficient for an agreeing "almost" state-independent utility. First, we make precise the notion of an "almost" state-independent utility. 
Consider a set of probability/state-dependent utility pairs $\left\{\left(p, U_{j}\right)\right\}$, each pair agreeing with the partial order $\prec$ for simple acts, according to (4.1).

Definition 31. Say that $\prec$ admits almost state-independent utilities for a set of $n$-rewards $\left\{r_{1}, \ldots, r_{n}\right\}$ if, for each $\varepsilon>0$, there exists a pair $\left(p, U_{j}\right)$ that agrees with $\prec$ on simple acts (and almost agrees, otherwise), where for a set of states $S^{\#}=\left\{s_{j 1}, \ldots, s_{j k}\right\}, p\left(S^{\#}\right) \geq 1-\varepsilon$,

$$
\max _{\substack{s_{j}, s_{j^{\prime}} \in S^{\#} \\ 1 \leq i \leq n}}\left|U_{j}\left(r_{i}\right)-U_{j^{\prime}}\left(r_{i}\right)\right| \leq \varepsilon .
$$

Say $\prec$ admits almost state-independent utilities if it does so for each set of $n$-rewards, $n=1, \ldots$.

Obviously, if $(p, U)$ agrees with $\prec$ and $U$ is state-independent, then $\prec$ admits almost state-independent utilities.

There are two problems created by state-dependent utilities. First, given the partial order $\prec$, we would like to indicate probability bounds for an event $E$ by $\prec$-preferences between a constant act of the form $H_{x}(s)=x B+$ $(1-x) W$ and the act $H_{E}(s)=B$ if $s \in E$, and $H_{E}(s)=W$ if $s \notin E$. That is, in general, we want the upper probability bound $p^{*}(E)$ to be the l.u.b.\{x: $H_{E} \prec H_{x}$ \} (or 1, if $H_{E} \sim \mathbf{B}$ ), and we want the lower bound, $p_{*}(E)$, to equal the g.l.b. $\left\{x: H_{x} \prec H_{E}\right\}$ (or 0, if $H_{E} \sim \mathbf{W}$ ). However, if such preferences are to indicate probability bounds, then we require that the rewards $B$ and $W$ carry state-independent utilities 1 and 0 , respectively. Thus the first problem.

Second, if a state $s_{j}$ is potentially null under $\prec$, then there are no $\prec$-preferences among pairs of acts called-off in case $s_{j}$ does not obtain. Let $\mathbf{H s}_{\mathbf{j}}$ be the family of $s_{j}$-called-off acts that yield outcome $W$ for all $s \notin s_{j}$. When $s_{j}$ is a potentially null state, $\forall\left(H_{1}, H_{2} \in \mathbf{H s}_{\mathbf{j}}\right), H_{1} \sim H_{2}$. Suppose $\precsim_{V}$ $(V \in \mathscr{V})$ extends $\prec$ (on simple acts) and let $\left\{\left(p, U_{j}\right)\right\}$ be the set of probability/(possibly) state-dependent utilities which represent $\precsim_{V}$ according to (4.1). Then, if state $s_{j}$ is potentially null under $\prec$, unfortunately, HL Axioms 1-4 impose too few restrictions on $U_{j}$ (the state-dependent utility, given state $s_{j}$ ) even when $p\left(s_{j}\right)>0$. In particular, it may be that $V\left(\mathbf{r}_{1}\right)>V\left(\mathbf{r}_{2}\right)$, yet for all the $U_{j}, U_{1}\left(r_{1}\right) \leq U_{1}\left(r_{2}\right)$.

To resolve both these problems, we impose a fifth axiom - a requirement of "stochastic dominance" among lotteries. For each state $s_{j}$ and each v.N-M lottery $L_{\alpha}$, define the set of acts $\left\{H_{j, m}^{\alpha}: H_{j, m}^{\alpha}(s)=\left(1-2^{-m}\right) W+\left(2^{-m}\right) L_{\alpha}\right.$, if $s \notin s_{j} ; H_{j, m}^{\alpha}\left(s_{j}\right)=L_{\alpha}$ for state $\left.s_{j}\right\} \quad(m=1, \ldots)$. Observe that, $(\forall j)$ $\lim _{m \rightarrow \infty}\left\{H_{j, m}^{\alpha}\right\}=H_{j, \alpha} \in \mathbf{H s}_{\mathbf{j}}$. Moreover, $H_{j, \alpha}\left(s_{j}\right)=L_{\alpha}$. Then, we require the following axiom:

HL Axiom 5. For each two "constant" acts $H_{L \alpha}(s)=L_{\alpha}$ and $H_{L \beta}(s)=L_{\beta}$,

$$
\forall(j, m)\left[H_{L \alpha} \prec H_{L \beta} \text { iff } H_{j, m}^{\alpha} \prec H_{j, m}^{\beta}\right] \quad(j=1, \ldots, n ; m=1, \ldots) .
$$


Thus, exactly when $\mathbf{L}_{\beta}$ is $\prec$-preferred to $\mathbf{L}_{\alpha}$ (as constant acts), HL Axiom 5 imposes a $\prec$-preference on sequences of pairs of lotteries, $\left(H_{j, m}^{\alpha}, H_{j, m}^{b}\right)$ which converge to the $s_{j}$-called-off pair $\left(H_{j, \alpha} ; H_{j, \beta}\right)$. Thus, we obtain the constraint (Definition 21 of the Appendix) “ $\neg\left(H_{j, \beta} \prec H_{j, \alpha}\right)$."

Lemma 4.2. Suppose $\prec$ satisfies HL Axioms 1-5. Then, for each $V \in \mathscr{V}$ (of Theorem 3.1) we may select (exactly) one pair $\left(p^{V}, U_{j}^{V}\right)$ from the set of pairs $\left\{\left(p, U_{j}\right)\right\}$ provided by Corollary 4.1 -where each pair represents $\precsim_{V}$ in accord with (4.1) - so that acts $\mathbf{W}$ and $\mathbf{B}$ have constant value and bound the state-dependent utilities of other rewards. In symbols,

$$
\begin{aligned}
\forall\left(s_{j}\right) \forall\left(L_{i}, L_{k} \in \mathbf{L}_{\mathbf{R}-\{W, B\}}\right), \quad H_{L_{i}} \prec H_{L_{k}} \\
\text { iff } 0=U_{j}^{V}(W) \leq U_{j}^{V}\left(L_{i}\right) \leq U_{j}^{V}\left(L_{k}\right) \leq U_{j}^{V}(B)=1,
\end{aligned}
$$

with at least one outside inequality strict for each $s_{j}$ such that $p\left(s_{j}\right)>0$, and all inequalities strict for each $s_{j}$ that is not $\prec$-potentially null.

Definition 32. We call $\left(p^{V}, U_{j}^{V}\right)$ the standard representation of $V$.

Thus, HL Axiom 5 (via HL Axiom 3) constrains state-dependent utilities of the rewards $\mathbf{W}$ and $\mathbf{B}$ in potentially null states, as desired. In the course of the proof of Theorem 6 (below), we explain how HL Axiom 5 also regulates the $\prec$-potentially null, state-dependent utilities of all v.N-M lotteries.

Of course, HL Axioms 1-5 are insufficient for guaranteeing existence of a state-independent utility agreeing with $\prec$. Counterexample 4.1 applies, that is, $\prec$ ' satisfies all five axioms (since no states are $\prec^{\prime}$-potentially null). However, as we show next, these axioms suffice for an almost state independent utility.

Theorem 6. Assume that $\prec$ satisfies HL Axioms 1-5.

(i) Then $\prec$ admits almost state-independent utilities.

(ii) If $\prec$ has a countable basis $\mathscr{B}$, each $\left(p, U_{j}\right)$ pair in Definition 31 agrees with $\prec$.

There is a sufficient condition for the existence of a state-independent utility over the finite set $\left\{W, B, r_{1}, \ldots, r_{i}, \ldots, r_{n}\right\}$, using closure (at one endpoint, at least) of the target sets $\mathscr{T}_{i}\left(r_{i}\right)$ defined in Definition 19.

LEMMA 4.3. If the target sets $\mathscr{T}_{i}\left(r_{i}\right)(i=1, \ldots, n)$ are not open intervals, there exists a subset $\mathscr{V}^{\prime} \subset \mathscr{V}$ of expected utilities for $\prec$ (agreeing on simple acts), where each $V^{\prime} \in \mathscr{V}^{\prime}$ is standardly represented by the set of pairs $\left\{\left(p^{\prime}, U_{j}^{\prime}\right)\right\}$ according to (4.1) and where $U_{j}^{\prime}\left(r_{i}\right)$ is state-independent $(i=$ $1, \ldots, n)$. 
Note: $\mathscr{V}^{\prime}$ may fail to be convex. Also, results similar to Lemma 4.3, using different assumptions, appear in Rios Insua (1992). Related ideas appear in Nau (1992).

5. Conditional preference and conditional probabilities. Let $e$ be an event. (Recall, we equate the set state $s_{j}$ with its elements.) Let $H_{1}$ and $H_{2}$ be a pair of $e$-called-off acts. Suppose $\prec$ satisfies HL Axioms 1 and 2 .

LEMMA 5.1. Let $H_{1}^{\prime}$ and $H_{2}^{\prime}$ be another pair of e-called-off acts which agree with $H_{1}$ and $H_{2}$ (respectively) on $e$, that is, $\forall(s \notin e),\left[H_{1}^{\prime}(s)=H_{2}^{\prime}(s)\right]$ and $\forall$ $(s \in e)$, $\left[H_{1}(s)=H_{1}^{\prime}(s)\right.$ and $\left.H_{2}(s)=H_{2}^{\prime}(s)\right]$ : (i) $H_{1} \prec H_{2}$ iff $H_{1}^{\prime} \prec H_{2}^{\prime}$ and (ii) $H_{1} \approx H_{2}$ iff $H_{1}^{\prime} \approx H_{2}^{\prime}$.

Therefore, a $\prec$-preference (or $\approx$-indifference) among two $e$-called-off acts does not depend upon how they are called-off, that is, the preference (or indifference) does not depend upon how the acts agree with each other when $e$ fails. This replicates the core of Savage's [(1954), page 23] "sure thing" postulate, $\mathrm{P} 2$, as that applies to our concept of a partially ordered preference.

Consider a (maximal) subset of $\mathbf{H}_{\mathbf{R}}$, denoted by $\mathbf{H}_{\mathbf{e}}$, where every two elements of $\mathbf{H}_{\mathbf{e}}$ form an e-called-off pair. Obviously, each such family $\mathbf{H}_{\mathbf{e}}$ of $e$-called-off acts is closed under convex combinations.

Definition 33. Define $\prec_{\mathbf{e}}=\prec / \mathbf{H}_{\mathbf{e}}$, the restriction of $\prec$ to the family of $e$-called-off acts in $\mathbf{H}_{\mathbf{e}}$. We call $\prec_{\mathbf{e}}$ the conditional $\prec$-preference relation, given $e$. (The preceding lemma insures this relation is well defined, that is, it depends on $e$ but not on how acts are called-off.)

Note: The event $e^{c}$ is essentially null with respect to the conditional preference $\prec_{\mathbf{e}}$.

Definition 34. Also, for each pair of horse lotteries $H_{1}$ and $H_{2}$, say that $H_{2}$ is $\prec$-preferred to $H_{1}$ given $e$, provided that, for some pair $H_{1}^{\prime}$ and $H_{2}^{\prime}$ (and by Lemma 5.1, provided for all pairs) of $e$-called-off acts agreeing (respectively) with $H_{1}$ and $H_{2}$ on $e, H_{1}^{\prime} \prec_{\mathbf{e}} H_{2}^{\prime}$.

In light of Lemma 5.1(i) and because $\mathbf{H}_{\mathbf{e}}$ is a subset of $\mathbf{H}_{\mathbf{R}}$, the following result is immediate.

Theorem 7. If $\prec\left(\right.$ over $\mathbf{H}_{\mathbf{R}}$ ) satisfies (a subset of) HL Axioms 1-5, then $\prec_{\mathbf{e}}\left(\right.$ over $\left.\mathbf{H}_{\mathbf{e}}\right)$ also satisfies the same horse lottery axioms, at least.

Theorem 7 prompts an interesting question: What is the relation between (i) the set of conditional probability/utility pairs $\left\{p(\mid e), U_{j \in e}\right\}$, given $e$, that arise from the representation of $\prec$ over the family of acts $\mathbf{H}_{\mathbf{R}}$ and (ii) the set of probability/utility pairs $\left\{p_{e}, U_{e, j \in e}\right\}$ that represent the conditional preference $\prec_{\mathbf{e}}$ over the restricted family of acts $\mathbf{H}_{\mathbf{e}}$ ? 
The following discussion of conditional indifference tells some of the answer.

DEFINITION 35. Let $\approx_{\mathbf{e}}$ be the conditional $\approx$-indifference relation, given $e$, defined by restricting $\approx$ to acts in the family $\mathbf{H}_{\mathbf{e}}$. Then, say that horse lotteries $H_{1}$ and $H_{2}$ are $\approx$-indifferent, given $e$, provided that for some pair $H_{1}^{\prime}$ and $H_{2}^{\prime}$ (and by Lemma 5.1, provided for all such pairs) of $e$-called-off acts agreeing (respectively) with $H_{1}$ and $H_{2}$ on $e, H_{1}^{\prime} \approx_{\mathbf{e}} H_{2}^{\prime}$.

It is important, however, to see that $\approx_{\mathrm{e}}$ is not always the same as the $\approx$-indifference relation (defined by Definition 8) induced by $\prec_{\mathbf{e}}$ over acts solely in $\mathbf{H}_{\mathbf{e}}$.

DEFINITION 36. Denote by $\approx_{\mathbf{H}_{\mathbf{e}}}$ the $\approx$-indifference relation over elements of $\mathbf{H}_{\mathbf{e}}$, induced by $\prec_{\mathbf{e}}$.

Of course $\approx_{\mathrm{e}}$-indifference entails $\approx_{\mathbf{H}_{\mathrm{e}}}$-indifference, but not conversely. $H_{1}$ and $H_{2}$ may be two $e$-called-off acts from a family $\mathbf{H}_{\mathbf{e}}$ which satisfies $H_{1} \approx_{\mathbf{H}_{\mathrm{e}}} H_{2}$, but where, nevertheless, $H_{1} \approx H_{2}$, that is, $H_{1} \approx_{\mathbf{e}} H_{2}$. We illustrate this phenomenon using a potentially null state which is not essentially null.

EXAMPLE 5.1. Consider a binary partition $S=\left\{s_{1}, s_{2}\right\}$ and horse lotteries defined over a binary reward set $\mathbf{R}=\{W, B\}$. Suppose $\prec$ is created by the Pareto principle applied to expected utility inequalities from the following set of probability/(state-independent) utility pairs: $S=\left\{(p, U): 1 \geq p\left(s_{1}\right) \geq 0.5\right.$; $U(B)>U(W)\}$. Then, $s_{2}$ is potentially null: acts are $\sim$-incomparable whenever they belong to a common $\mathbf{H}_{\mathbf{S}_{2}}$ family. [With $p\left(s_{1}\right)=1$, all elements of $\mathbf{H}_{\mathbf{S}_{2}}$ have equal expected utility.] Hence, $\prec_{\mathbf{S}_{2}}$ is vacuous. So, based on $\prec_{\mathbf{S}_{2}}$ restricted to a family $\mathbf{H}_{\mathbf{S}_{2}}$, all pairs of (e-called-off) acts are $\approx_{\mathbf{H}_{\mathbf{S} 2}}$-indifferent. However, the pair of $s_{2}$-called-off acts $\left(H_{1}, H_{2}\right)$, defined by $H_{1}\left(s_{1}\right)=H_{2}\left(s_{1}\right)=$ $W, H_{1}\left(s_{2}\right)=W$ and $H_{2}\left(s_{2}\right)=B$, though $\sim$-incomparable are not $\approx-$ indifferent: $H_{1} \sim H_{2}$ and $H_{1} \approx H_{2}$. This is shown as follows. Consider the act $H_{3}$ defined by $H_{3}\left(s_{1}\right)=B$ and $H_{3}\left(s_{2}\right)=W$. Observe that $0.5 H_{1}+0.5 H_{3} \sim$ $0.7 \mathrm{~W}+0.3 B$, whereas $0.7 \mathrm{~W}+0.3 B \prec 0.5 \mathrm{~W}+0.5 B=0.5 H_{2}+0.5 H_{3}$. This shows that $H_{1} \approx H_{2}$.

Returning to the question, above, we state our central result about conditional probabilities and conditional preferences.

THEOREM 8. (i) If $\left(p, U_{j}\right)$ belongs to the set of probability/utility pairs representing $\prec$, then the pair $\left(p(\mid e), U_{j \in e}\right)$ belongs to the set that represent the conditional preference $\prec_{\mathbf{e}}$.

(ii) Suppose that the pair $\left(p_{e}, U_{e, j \in e}\right)$ belongs to the set representing the conditional preference $\prec_{\mathbf{e}}$ with respect to the family $\mathbf{H}_{\mathbf{e}}$. Then for some pair $\left(p, U_{j}\right)$ in the set that represents $\prec, p(\mid e)=p_{e}$ and $U_{j \in e}=U_{e, j \in e}$, provided two conditions obtain: (1) The event $e$ is not potentially null (with respect 
to $\prec$ ) and (2) the expected utility $V_{\mathbf{e}}\left(\right.$ ) (with arguments from $\mathbf{H}_{\mathbf{e}}$ ), corresponding to the pair $\left(p_{e}, U_{e, j \in e}\right)$, does not use $\prec-p r e c l u d e d$ target endpoints, as regulated by Definition 21 (of the Appendix).

Next, we offer an example of Theorem 8, relating Bayes' updating to conditional preferences.

ExAMPLE 5.2. Consider a partition into three states $\left\{s_{1}, s_{2}, s_{3}\right\}$ and acts involving the three rewards, $W, r$ and $B$. Let $\mathbf{r}$ denote the constant act, with outcome $r$ in each state. For $j=1,2$ and 3, define the three acts $H_{j}\left(s_{j}\right)=B$, $H_{j}\left(s_{k}\right)=W(j \neq k)$ and also the three acts $H_{j, r}\left(s_{j}\right)=r$ and $H_{j, r}\left(s_{k}\right)=W$ $(j \neq k)$. Apart from the strict preferences that follow because $\mathbf{W}$ and $\mathbf{B}$ are, respectively, the "worst" and "best" acts, suppose also the agent reports these preferences:

$$
0.5 \mathrm{~W}+0.5 H_{3, r} \prec H_{1} \prec H_{3, r} \prec H_{2} \prec H_{3} \prec \mathbf{r} \prec 0.5 H_{3}+0.5 \mathbf{r} .
$$

We investigate the standardized, state-independent utility representations for these preferences. That is, with $U(W)=0, U(B)=1$, let $u=U(r)$, independent of the state $s_{j}$. If we denote by $p_{j}$ the probability of state $s_{j}$, then the preferences above are modeled by each probability/utility pair $\left(p_{1}, p_{2}, p_{3} ; u\right)$ satisfying $0<0.5 p_{3} u<p_{1}<p_{3} u<p_{2}<u<0.5 p_{3}+0.5$.

For each $0<u<1$, it is possible to determine the set $\mathscr{P}(u)$ of all $\left(p_{1}, p_{2}, p_{3}\right)$ that satisfy these inequalities. For example, the set $\mathscr{P}(0.5)$ is shown in Figure 2. The union of all sets $\mathscr{P}(u) \times\{u\}$ such that $\mathscr{P}(u) \neq \varnothing$ is the set of all probability/utility pairs that agree with the strict preferences above. From this set, one can determine other preferences not listed above which must also hold if the axioms do. For example, it is required, though not obvious from the reported preferences, that $0.4 B+0.6 \mathrm{~W} \prec \mathbf{r}$. [By contrast, it is obvious from the preferences above that $(1 / 3) \mathbf{B}+(2 / 3) \mathbf{W} \prec \mathbf{r}$.]

If we were to learn that, say, the event $E=\left\{s_{1}, s_{2}\right\}$ occurred, we can determine which preferences are implied in the conditional problem. The set of all pairs $\left(q_{1}, u\right)$, where $q_{1}$ is a conditional probability of $s_{1}$ given $E$, is shown in Figure 3. Observe that, as provided by Theorem 8, the set of conditional probabilities from Figure 2 is exactly the set represented by the vertical line (at $u=0.5$ ) in Figure 3. However, the set shown in Figure 3 is not convex since it contains the points $(0.415,0.293)$ and $(0.455,0.379)$, but does not contain the point $(0.435,0.336)=0.5(0.415,0.293)+$ $0.5(0.455,0.379)$.

6. Concluding remarks. There is a burgeoning literature dealing with applications of sets of probabilities. Separate from work on robust Bayesian statistical analysis, they occur also in the following settings: as a rival account to strict Bayesian theory for representing uncertainty, such as in Levi's $(1974,1980)$ theory for Ellsberg's (1961) "paradox"; relating to indeterminate degrees of belief, as in Smith's (1961) theory of "medial odds" developed by Williams (1976), Giron and Rios (1980), Walley (1991) and Nau 
$(0,1,0)$

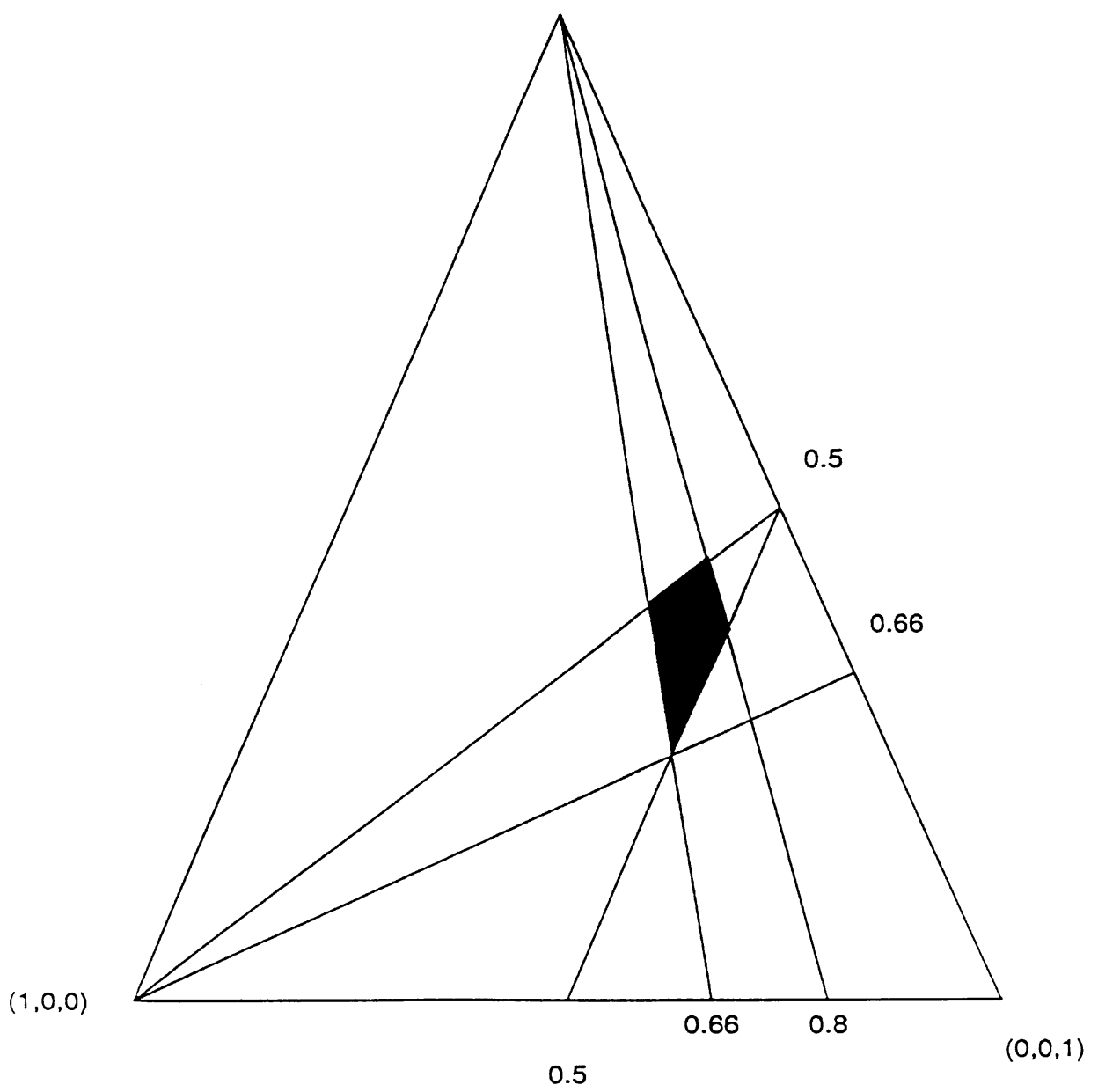

Fig. 2. The set $P(0.5)$ in Example 5.2.

(1993); and as a method for capturing multiple "expert" opinions [Kadane and Sedransk (1980); Kadane (1986)]. In addition, sets of probabilities arise from incomplete elicitations, where some but not all of an agent's opinions are formalized by inequalities in probabilities and the question is what decisions are fixed by these partially reported degrees of belief; see Moskowitz, Wong and Chu (1988) and White (1986). Dual to sets of probabilities, the articles by Aumann (1962) and Kannai (1963) explore the existence of ("linear") utilities for von Neumann-Morgenstern lotteries when probabilities are completely specified.

However, these efforts rely on convexity of the spaces of probabilities and utilities to arrive at their conclusions. From our point of view, this mathemat- 


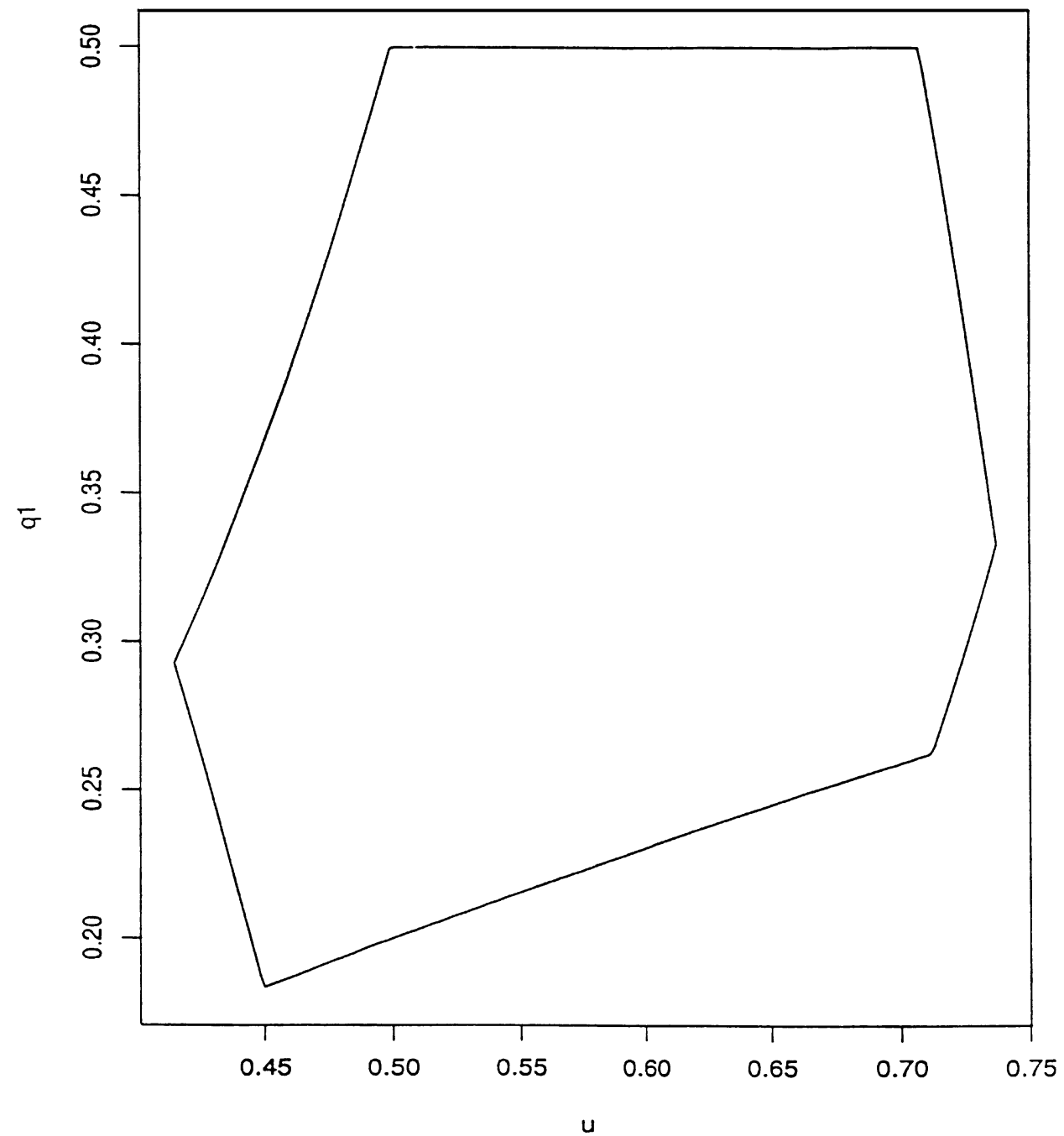

FIG. 3. The set of conditional probabilities and utilities.

ical convenience is justified under an assumption, for example, that at least one of the agent's probability and utility is fully determinate. For instance, in light of Corollary 3.4, convexity is appropriate for Bayesian robustness when a loss function is specified but probability is left indeterminate. Likewise, our theory endorses the use of a convex set of utilities given a determinate probability, as in Aumann's (1962) result concerning existence of a utility agreeing with a partially ordered preference over simple von Neumann-Morgenstern lotteries. However, as shown by Theorem 1, 
partially ordered preferences that obey a (weak) Pareto condition may not admit a convex (or even connected) set of agreeing probability/utility pairs. One way to require convexity of the agreeing sets, then, is to restrict the scope of the Pareto condition [see Levi (1990)], but that is a move we are not willing to make.

Corollary 3.4 prompts a serious question, we think, about the extent to which our proof technique for extending a partially ordered preference is useful for the representation theorems of this essay. To wit, since the set $\mathscr{V}$ of agreeing "linear" utilities in Theorem 5 is convex, why bother with the elaborate inductive argument only to arrive at what "separating hyperplanes" yields directly? The answer has two parts.

As a first reason, Theorem 5 applies without additional topological assumptions about the relation $\prec$. Specifically, to the best of our knowledge, all the existing theorems that appeal to "separating hyperplanes" in order to provide necessary and sufficient conditions for representing a partially ordered strict preference relation $\prec$ by a convex set of "linear" utilities or by a convex set of probabilities, make assumptions regarding the boundaries of $\prec$. Otherwise, for results that are based on a partially ordered weak preference relation $\precsim$, whether preference at the boundary of $\mathscr{V}$ is strict or not, is not determined by such an approach.

For an illustration of the former approach, Walley [(1991), Section 3.7.8, condition R8] requires that strict preference over gambles be "open" so that so-called strong separation leads to a representation in terms of sets of probabilities "closed" with respect to infimums. By avoiding "separating hyperplanes," we are able to sidestep this artifice. Surfaces of the set $\mathscr{V}$ need not have a simple topological character.

For an illustration of the latter approach, Giron and Rios (1980) use a reflexive, partial (quasi-Bayesian) preference relation, denoted in their paper by $\lesssim$, which they represent with a closed, convex set of probabilities. They note [Giron and Rios (1980), footnote 3, page 20] that their method generates the same "quasi-Bayesian preorder" whether the so-called uncertainty set of probabilities (which they denote by $K^{*}$ ) or its closure $\left(\bar{K}^{*}\right)$ is used. To explain our assertion about the loss of information at the boundary of $\mathscr{V}$, consider the following example involving preferences over acts using only two prizes, $\mathbf{W}$ and $\mathbf{B}$.

Example 6.1. Define act $H_{E}$ as $H_{E}(s)=B$ for $s \in E$, and $H_{E}(s)=W$ otherwise.

Case 1. The agent reports the strict preferences $x B+(1-x) W \prec H_{E}$ for $0<x \leq 0.6$ and noncomparability $x B+(1-x) W \sim H_{E}$ for $0.6<x \leq 1$.

Case 2. The agent reports the strict preferences $x B+(1-x) W \prec H_{E}$ for $0<x<0.6$ and noncomparability $x B+(1-x) W \sim H_{E}$ for $0.6 \leq x \leq 1$.

The (closed) target set of utilities for $H_{E}$ is the same in both cases: $\mathscr{T}\left(H_{E}\right)=[0.6,1]$. However, in the first case the lower bound is not a "candidate utility" (Definition 24), whereas in the second case it is. Therefore, by our construction, the representation for the agent's strict preferences in Case 
1 is the set $\mathscr{P}=\{P: 0.6<P(E) \leq 1\}$ and in the second case it is the closed set $\overline{\mathscr{P}}=\{P: 0.6 \leq P(E) \leq 1\}$.

By contrast, the Giron and Rios (1980) theory uses only a weak preference relation, $\precsim$. In both Cases 1 and 2 their theory entails

$$
\begin{array}{ll}
x B+(1-x) W \precsim H_{E} & \text { for } 0<x \leq 0.6 \text { and } \\
x B+(1-x) W \sim H_{E} & \text { for } 0.6<x \leq 1 .
\end{array}
$$

[The weak preferences of Case 2 result from applying Giron and Rios' Axiom A5 (continuity). In particular, that axiom yields the conclusion $0.6 B+$ $0.4 W \precsim H_{E}$ from the premise $x B+(1-x) W \precsim H_{E}(0<x<0.6)$.] In their notation, the weak-preference relation does not distinguish between these two cases: where $\left.K^{*}=\{p: 0.6<p(E) \leq 1\}\right)$ and $\bar{K}^{*}=\{p: 0.6 \leq p(E) \leq 1\}$, though our strict-preference does.

As a second reason for bypassing proof techniques using "separating hyperplanes," though the set $\mathscr{V}$ is convex, not so for the set of "linear" utilities that admit a decomposition as subjective (almost) state-independent utilities. We do not see how to show the existence of the set of agreeing probability/(almost) state-independent utility pairs, corresponding to Theorem 6, without exploring details about the surface of $\mathscr{V}$. In light of Theorem 5 , we have no right to assume those surfaces are closed. By contrast, when $\mathscr{V}$ has sufficiently many closed faces, Lemma 4.3 gives a representation of $\prec$ in terms of sets of probability/state-independent utility pairs. Thus, we feel justified in our choice of an "inductive" proof technique by the increased content to the theorems reached.

\section{APPENDIX}

\section{Proofs of selected results.}

A. Results from Section 2. Corollaries 2.1, 2.2 and 2.3 have elementary proofs.

Proof of Corollary 2.4. From left to right, argue indirectly and apply Corollary 2.3 for a contradiction. In the other direction, assume that $x H_{1}$ $+(1-x) H \approx x H_{2}+(1-x) H$ for some $1 \geq x>0$ and some lottery $H$. Also, assume $y H_{1}+(1-y) H_{3} \prec(\succ) H_{4}$, with $1 \geq y>0$. Then by HL Axiom 2, $\forall(1 \geq z>0), \quad z\left(y H_{1}+(1-y) H_{3}\right)+(1-z) H \prec(\succ) z H_{4}+(1-z) H$. Let $z * /(x+y-x y)>0$ and then $1>z$ (unless $x=y=1$, in which case we are done). Last, define the term $w=y /(x+y-x y)$ and we know that $0<w<1$. Thus, we have $w\left(x H_{1}+(1-x) H\right)+(1-w) H_{3} \prec(\succ) z H_{4}+(1-z) H$. Since $\left(x H_{1}+(1-x) H\right) \approx\left(x H_{2}+(1-x) H\right)$, by Corollary 2.3 also we have $w\left(x H_{2}+(1-x) H\right)+(1-w) H_{3} \prec(\succ) z H_{4}+(1-z) H$. Again by HL Axiom 2 , we may cancel the common factor $(1-z) H$ from both sides, to yield $y H_{2}+(1-y) H_{3} \prec(\succ) H_{4}$. By Corollary $2.3, H_{1} \approx H_{2}$. 
Proof of Corollary 2.5. Assume the premises and, by Corollary 2.3, show using HL Axiom 3 that $\forall\left(0<x \leq 1, H_{a}, H_{b}\right)$ whenever $x M+(1-$ $x) H_{a} \prec(\succ) H_{b}$, then $x H+(1-x) H_{a} \prec(\succ) H_{b}$.

Lemma 2.1 has a straightforward proof.

Proof of Lemma 2.2. Without loss of generality, as $L$ is discrete, write $L$ as $\left\{P\left(r_{n}\right): P\left(r_{i}\right) \geq P\left(r_{j}\right)\right.$ for $\left.i \leq j\right\}$. Let

$$
x_{n}=\sum_{i=1}^{n} P\left(r_{i}\right)
$$

and define the simple lotteries $L_{n}=\left\{\left(1 / x_{n}\right) P\left(r_{i}\right): i=1, \ldots, n\right\}$. Then $\left\{H_{L_{n}}\right\}$ $\Rightarrow H_{L}$. If for each $r_{n} \in \operatorname{supp}(L), \mathbf{r}_{\mathbf{n}} \prec \mathbf{r}$, then by HL Axioms 1 and $2, H_{L_{n}} \prec \mathbf{r}$ (or, if $\mathbf{r} \prec \mathbf{r}_{n}$, then $\mathbf{r} \prec H_{L_{n}}$ ). Thus, we have the desired conclusion: not $\left(\mathbf{r} \prec H_{L}\right)$ [or, alternatively, not $\left(H_{L} \prec \mathbf{r}\right)$ ]. For if not, by HL Axiom 3 and transitivity of $\prec,\left(H_{L} \prec H_{L}\right)$.

Proof of Corollary 2.6. On the contrary, if a utility $V$ for acts is unbounded, then there are acts with infinite utility.

Just consider the discrete horse lottery $H_{\infty}$, where $H_{\infty}\left(s_{j}\right)=\sum_{i}\left(2^{-i}\right) P_{i, j}$ for a sequence of acts $H_{i}$ such that $V\left(H_{i}\right) \geq 2^{i}(i=1, \ldots)$. Then, by the expected utility property, $V\left(H_{\infty}\right)=\infty$. The existence of such acts leads to a contradiction with the first two axioms, just as in the St. Petersburg paradox. Assume for convenience that $H_{1} \prec H_{2}$. By axiom HL Axiom 2, $0.5 H_{1}+0.5 H_{\infty} \prec 0.5 H_{2}$ $+0.5 H_{\infty}$. However, $V\left(0.5 H_{1}+0.5 H_{\infty}\right)=V\left(0.5 H_{2}+0.5 H_{\infty}\right)=V\left(H_{\infty}\right)=\infty$, which, if $V$ agrees with $\prec$, entails the contrary result that $0.5 H_{1}+0.5 H_{\infty} \sim$ $0.5 H_{2}+0.5 H_{\infty}$.

Proof of Lemma 2.3. The proof is indirect. Most of the work is done by HL Axiom 3. We present the argument for the case in which $\prec$ fails to be bounded above, using Axiom 3(b). By similar reasoning using Axiom 3(a) instead, the result obtains when $\prec$ fails to be bounded below.

Let $\left\{H_{n}: n=1, \ldots\right\}$ be an increasing chain and suppose it is not bounded above, that is, $\lim _{n \rightarrow \infty} \sup \left\{x:\left(H_{2} \prec x H_{1}+(1-x) H_{n}\right)\right\}=1$. Choose a subsequence, also denoted by $\left\{H_{n}\right\}$, so that $x_{n} \geq 1-1 / n$ and so that $H_{2} \prec x_{n} H_{1}+$ $\left(1-x_{n}\right) H_{n}$. However, $\left\{x_{n} H_{1}+\left(1-x_{n}\right) H_{n}\right\} \Rightarrow H_{1}$. Trivially, the constant sequence $\left\{H_{2}\right\} \Rightarrow H_{2}$. Also, $H_{1} \prec H_{2}$ by assumption. Then by HL Axiom 3(b), $H_{1} \prec H_{1}$, contradicting HL Axiom 1 .

Proof OF CoRollary 2.7. If not, then there is an unbounded increasing (or decreasing) $\prec_{W}$-chain of preferences amongst the set of rewards $\mathbf{R}$. By Lemma 2.3, $\prec_{W}$ does not satisfy both Axioms 1 and 3.

Proof of Corollary 2.8. Let $H_{1}$ and $H_{2}$ be a pair of acts which are "called-off" in case $\mathbf{n}$ does not obtain, that is, $\forall(s \notin \mathbf{n}), H_{1}(s)=H_{2}(s)$. 
(Properties of "called-off" acts are examined in Section 5.) Define $k$ pairs of acts "called-off" in case $s_{j_{i}}$ obtains, $H_{1_{i}}$ and $H_{2_{i}}(i=1, \ldots, k)$ as follows: Let $l$ be a lottery. $\forall\left(s \in s_{j_{i}}\right),\left[{ }^{2} H_{1_{i}}(s)=H_{1}(s)\right.$ and $\left.H_{2_{i}}(s)=H_{2}(s)\right] ; \forall(s \in \mathbf{n} \& s \notin$ $\left.s_{j_{i}}\right),\left[H_{1_{i}}(s)=H_{2_{i}}(s) \stackrel{j_{i}}{=} L\right] ; \forall(s \notin \mathbf{n}),\left[H_{1_{i}}(s)=H_{2_{i}}(s)=H_{1}(s)=H_{2}(s)\right]$. By assumption, each $s_{j_{i}} \in \mathbf{n}$ is essentially null. Therefore, by iteration of Corollary 2.4 (and transitivity of $\approx$ ) $H_{1}^{\prime} \approx H_{2}^{\prime}$, where $H_{1}^{\prime}=\sum_{i=1}^{k}(1 / k) H_{1_{i}}$ and $H_{2}^{\prime}=\sum_{i=1}^{k}(1 / k) H_{2_{i}}$. However, $H_{1}^{\prime}=(1 / k) H_{1}+(k-1 / k) H$ and, likewise, $H_{2}^{\prime}=(1 / k) H_{2}+(k-1 / k) H$ for act $H$ defined by $\forall(s \in \mathbf{n}),[H(s)=L]$ and $\forall(s \notin \mathbf{n})\left[H(s)=H_{1}(s)=H_{2}(s)\right]$. Then, by Corollary 2.4, the desired result obtains, $H_{1} \approx H_{2}$.

B. Proof of Theorem 2. The extension from $\prec$ to $\prec$ ' is given in steps, by adding the two new rewards one at a time. First, extend $\prec$ to a partial order $\prec *$ on $\mathbf{H}_{\mathbf{R} \cup\{W\}}$, where $W$ is left $\sim{ }^{*}$-incomparable with elements of $\mathbf{H}_{\mathbf{R}}$. The definition of $\prec *$ is introduced by a lemma that shows the extension is minimal.

Lemma 2.4. Suppose $H_{1}, H_{2}, H_{1}^{\prime}$ and $H_{2}^{\prime} \in \mathbf{H}_{\mathbf{R}}$ and are related as follows: $H_{1}\left(s_{j}\right)=x_{j} L_{j}+\left(1-x_{j}\right) L_{1, j}$ and $H_{2}\left(s_{j}\right)=x_{j} L_{j}+\left(1-x_{j}\right) L_{2, j}$, while $H_{1}^{\prime}\left(s_{j}\right)=$ $x_{j} L_{j}^{\prime}+\left(1-x_{j}\right) L_{1, j}$ and $H_{2}^{\prime}\left(s_{j}\right)=x_{j} L_{j}^{\prime}+\left(1-x_{j}\right) L_{2, j}$. Then $H_{1} \prec H_{2}$ iff $H_{1}^{\prime} \prec$ $H_{2}^{\prime}$.

Proof. The lemma is immediate by HL Axiom 2 and the identity $0.5 H_{1}+$ $0.5 H_{2}^{\prime}=0.5 H_{2}+0.5 H_{1}^{\prime}$.

Now, let $H_{i} \in \mathbf{H}_{\mathbf{R} \cup\{W\}}$ be written $H_{i}\left(s_{j}\right)=x_{i, j} W+\left(1-x_{i, j}\right) L_{i, j}$, where $L_{i, j} \in \mathbf{H}_{\mathbf{R}}$ is well defined if and only if $x_{i, j}<1$. Choose a reward $r \in \mathbf{R}$ and let $H_{i}^{\#} \in \mathbf{H}_{\mathbf{R}}$ be the act that results by substituting $r$ for $W$ in $H_{i}$. Thus, $H_{i}^{\#}\left(s_{j}\right)=x_{i, j} r+\left(1-x_{i, j}\right) L_{i, j}$. Lemma 2.4 shows this choice is arbitrary and, if $\prec *$ is to extend $\prec$, it must satisfy the following:

Definition $15(\prec *)$. Given $H_{1}, H_{2} \in \mathbf{H}_{\mathbf{R} \cup\{W\}}$, as expressed above, define the preference $\prec *$ from $\prec$ by $H_{1} \prec^{*} H_{2}$ iff $x_{1, j}=x_{2, j}$ (for all $s_{j} \notin \mathbf{n}$ ) and $H_{1}^{\#} \prec H_{2}^{\#}$.

LEMMA 2.5. The order $\prec *$ is identical with $\prec$ on $\mathbf{H}_{\mathbf{R}}$ and satisfies $H L$ Axioms 1-3.

Proof. If $H_{1}, H_{2} \in \mathbf{H}_{\mathbf{R}}$, then $x_{1, j}=x_{2, j}=0, H_{1}=H_{1}^{\#}, H_{2}=H_{2}^{\#}$ and thus $H_{1} \prec * H_{2}$ iff $H_{1} \prec H_{2}$. Next, we show that $\prec *$ satisfies the axioms. Consider all $H_{i} \in \mathbf{H}_{\mathbf{R} \cup\{W\}}$.

$H L$ Axiom 1 (irreflexivity). If, on the contrary, for some $H_{1}, H_{1} \prec * H_{1}$, then $H_{1}^{\#} \prec H_{1}^{\#}$, contradicting the irreflexivity of $\prec$.

Transitivity holds because if $H_{1} \prec * H_{2}$ and $H_{2} \prec * H_{3}$, then the corresponding three $H_{i}^{\#}$ acts $(i=1,2,3)$ can be written $x_{j}(r)+\left(1-x_{j}\right) L_{i, j}$. Since $\prec$ is transitive, $H_{1}^{\#} \prec H_{3}^{\#}$; thus, $H_{1} \prec{ }^{*} H_{3}$. 
HL Axiom 2 (independence). $\forall(0<y \leq 1), \forall H \in \mathbf{H}_{\mathbf{R} \cup\{W\}} H_{1} \prec * H_{2}$ iff $x_{1, j}=x_{2, j}$ and $H_{1}^{\#} \prec H_{2}^{\#}$ iff $y x_{1, j}+(1-y) x_{3, j}=y x_{2, j}+(1-y) x_{3, j}$ and $y H_{1}^{\#}+(1-y) H_{3}^{\#} \prec y H_{2}^{\#}+(1-y) H_{3}^{\#}$ iff $y H_{1}+(1-y) H_{3} \prec * y H_{2}+$ $(1-y) H_{3}$.

$H L$ Axiom 3. Let $\left\{H_{1 n} \prec * H_{2 n}\right\}$ be an infinite sequence of $\prec *$-preferences where $\left\{H_{1 n}\right\} \Rightarrow H_{1},\left\{H_{2 n}\right\} \Rightarrow H_{2}$ and assume $H_{2} \prec * H_{3}$. Thus $H_{1 n}^{\#} \prec H_{2 n}^{\#}$, where $\left\{H_{1 n}^{\#}\right\} \Rightarrow H_{1}^{\#}$ and $\left\{H_{2 n}^{\#}\right\} \Rightarrow H_{2}^{\#}$. Since $H_{2} \prec * H_{3}$, then $H_{2}^{\#} \prec H_{3}^{\#}$. By applying HL Axiom 3 to these $\prec$-preferences, we obtain $H_{1}^{\#} \prec H_{3}^{\#}$. We derive $x_{1, j}=x_{3, j}$ from the equalities $x_{1, j}^{n}=x_{2, j}^{n}$ and $x_{2, j}=x_{3, j}$. Therefore, $H_{1} \prec{ }^{*} H_{3}$. The argument for HL Axiom 3(b) is similar.

Lemma 2.5 shows, also, that if $\prec$ ' is defined on $\mathbf{R} \cup\{W\}$, extends $\prec$ and satisfies the axioms, then it extends $\prec *$. That is, $\prec *$ is a minimal extension of $\prec$ to the domain $\mathbf{R} \cup\{W\}$. Next, we extend $\prec^{*}$ to a preference $\prec_{W}$ in which $\mathbf{W}$ serves as a least preferred (worst) act. (The reader is alerted to the fact that, though the partial order $\prec_{W}$ makes $\mathbf{W}$ a least preferred act with respect to elements of $\mathbf{H}_{\mathbf{R}}$, it does not guarantee that $W$ is, state by state, a least favorable reward. This feature is addressed in Section 4.1, where we consider state-dependent utilities for partial orders.)

Definition $16\left(\prec_{W}\right) . \quad \forall\left(H_{1}, H_{2} \in \mathbf{H}_{\mathbf{R} \cup\{W\}}\right), H_{1} \prec_{\mathbf{w}} H_{2}$ iff either (a) $H_{1} \prec * H_{2}$ or (b) $\exists\left\{H_{n} \in \mathbf{H}_{\mathbf{R}}\right\}, \exists\left\{H_{1 n}\right\} \Rightarrow H_{1}, \exists\left\{H_{2 n}\right\} \Rightarrow H_{2}$ and $\exists\left(q_{n}\right.$ : $\left.0.5 \leq q_{n}<1\right)$ with $\lim _{n \rightarrow \infty}\left\{q_{n}\right\}=q, q<1$, such that $\forall n, q_{n} H_{1 n}+(1-$ $\left.q_{n}\right) H_{n} \prec *($ or $\approx *) q_{n} H_{2 n}+\left(1-q_{n}\right) \mathbf{W}$.

LEMMA 2.6. (1) The partial order $\prec_{W}$ agrees with $\prec$ on $\mathbf{H}_{\mathbf{R}}$.

(2) $\mathbf{W}$ bounds $\prec *$ from below; that is, $\forall\left(H \in \mathbf{H}_{\mathbf{R}}\right), \mathbf{W} \prec_{W} H$.

(3) $\prec_{W}$ satisfies HL Axioms 1-3.

Proof. (1) Let $H_{1}$ and $H_{2}$ belong to $\mathbf{H}_{\mathbf{R}}$. If $H_{1} \prec H_{2}$, then by Lemma 2.4, $H_{1} \prec * H_{2}$, and by clause (a) in Definition 16, $H_{1} \prec_{W} H_{2}$. For the converse, if $H_{1} \prec_{W} H_{2}$, then it is not by clause (b), since $\forall\left(H_{n} \in \mathbf{H}_{\mathbf{R}}\right)$ and for all sufficiently large $n$, as $1>q \geq 0.5, q_{n} H_{1 n}+\left(1-q_{n}\right) H_{n} \sim^{*}$ (and $\neq^{*}$ ) $q_{n} H_{2 n}+\left(1-q_{n}\right) \mathbf{W}$. Hence, it must be that clause (a) obtains. So, $H_{1} \prec * H_{2}$ and $H_{1} \prec H_{2}$.

(2) For each $H \in \mathbf{H}_{\mathbf{R}}$, recall that $0.5 H+0.5 \mathbf{W} \approx * 0.5 \mathbf{W}+0.5 H$. Then, in Definition 16(b), set $H_{1}=\left\{H_{1 n}\right\}=\mathbf{W}, H_{2}=\left\{H_{2 n}\right\}=H, H_{n}=H$ and $q_{n}=0.5$. Thus, $\mathbf{W} \prec_{W} H$.

(3) We verify the axioms individually:

$H L$ Axiom 1 (irreflexivity). On the contrary, suppose that $H_{1} \prec_{W} H_{1}$. There are two cases to consider. If this $\prec_{W}$-relation results by Definition 16(a), then $H_{1} \prec * H_{1}$, contradicting Lemma 2.4. If we hypothesize that $H_{1} \prec_{W} H_{1}$ results by Definitions $16(\mathrm{~b})$, then we derive a contradiction as follows. Let $H_{1 n}^{\prime}=q_{n} H_{1 n}+\left(1-q_{n}\right) H_{n}$ and $H_{2 n}^{\prime}=q_{n} H_{2 n}+\left(1-q_{n}\right) \mathbf{W}$. A necessary condition for the relation $H_{1 n}^{\prime} \prec *\left(\right.$ or $\left.\approx^{*}\right) H_{2 n}^{\prime}$ to obtain is that $x_{H_{1 n}^{\prime}}=x_{H_{2 n}^{\prime}}$ 
(except on essentially null states). However, as both $\left\{H_{1 n}\right\} \Rightarrow H_{1}$ and $\left\{H_{2 n}\right\} \Rightarrow$ $H_{1}$, while $\lim _{n \rightarrow \infty} q_{n}=q<1$, this is impossible. That is, $\lim _{n \rightarrow \infty} x_{H_{1 n}^{\prime}}=$ $\lim _{n \rightarrow \infty} q_{n} x_{H_{1 n}}=0$, while $x_{H_{2 n}^{\prime}} \geq\left(1-q_{n}\right)$; hence, for all sufficiently large $n$, $x_{H_{1 n}^{\prime}}<x_{H_{2 n}^{\prime}}$.

HL Axiom 1 (transitivity). Suppose both $H_{1} \prec_{W} H_{2}$ and $H_{2} \prec_{W} H_{3}$ obtain. There are four cases to consider depending upon which clause in Definition 16 is used for each $\prec_{W}$-preference. The argument is most complicated when Definition 16(b) is used twice; hence, we give the details for this case only. Thus, assume there are two sequences of *-relations:

$$
H_{1 n}^{\prime} \prec *\left(\text { or } \approx^{*}\right) H_{2 n}^{\prime} \text { and } H_{2 n}^{\prime \prime} \prec *\left(\text { or } \approx^{*}\right) H_{3 n}^{\prime}
$$

where

$$
\begin{array}{ll}
H_{1 n}^{\prime}=q_{n} H_{1 n}+\left(1-q_{n}\right) H_{n}, & H_{2 n}^{\prime}=q_{n} H_{2 n}+\left(1-q_{n}\right) \mathbf{W}, \\
H_{2 n}^{\prime \prime}=q_{n}^{\prime} \hat{H}_{2 n}+\left(1-q_{n}^{\prime}\right) H_{n}^{\prime}, & H_{3 n}^{\prime}=q_{n}^{\prime} H_{3 n}+\left(1-q_{n}^{\prime}\right) \mathbf{W}
\end{array}
$$

and where $\left\{H_{1 n}\right\} \Rightarrow H_{1}, \quad\left\{H_{2 n}\right\} \Rightarrow H_{2}, \quad\left\{\hat{H}_{2 n}\right\} \Rightarrow H_{2}, \quad\left\{H_{3 n}\right\} \Rightarrow H_{3}$ and $\lim _{n \rightarrow \infty}\left\{q_{n}\right\}=q, \lim _{n \rightarrow \infty}\left\{q_{n}^{\prime}\right\}=q^{\prime}$, with $0.5 \leq q, q^{\prime}<1$. Then $\forall\left(0<r_{n}<1\right)$, $r_{n} H_{1 n}^{\prime}+\left(1-r_{n}\right) H_{2 n}^{\prime \prime}{ }^{*}$ (or $\left.\approx *\right) r_{n} H_{2 n}^{\prime}+\left(1-r_{n}\right) H_{3 n}^{\prime}$. This is an $\prec^{*}$ preference, unless both equations of (B1) are $\approx^{*}$-indifferences. Choose $r_{n}$ so that $r_{n} q_{n}=\left(1-r_{n}\right) q_{n}^{\prime}$. Since $\left\{H_{2 n}\right\}$ and $\left\{\hat{H}_{2 n}\right\}$ both converge to $H_{2}$, by HL Axiom 2 , cancel the common acts in $H_{2 n}^{\prime}$ and $H_{2 n}^{\prime \prime}$ (also common with acts in $H_{2}$ ). Then apply clause Definition 16(b) to obtain $H_{1} \prec_{W} H_{3}$.

$H L$ Axiom 2 (independence). $\forall H \in \mathbf{H}_{\mathbf{R} \cup\{\mathbf{w}\}}, \forall 0<x \leq 1$ :

Case (a). $H_{1} \prec^{*} H_{2}$ iff $x H_{1}+(1-x) H \prec * x H_{2}+(1-x) H$ iff $x H_{1}+$ $(1-x) H \prec_{W} x H_{2}+(1-x) H$.

Case (b)-(i). If $q_{n} H_{1 n}+\left(1-q_{n}\right) H_{n} \prec *\left(\right.$ or $\left.\approx^{*}\right) q_{n} H_{2 n}+\left(1-q_{n}\right) \mathbf{W}$, then $r_{n}\left[q_{n} H_{1 n}+\left(1-q_{n}\right) H_{n}\right]+\left(1-r_{n}\right) H \prec * \quad($ or $\approx *) r_{n}\left[q_{n} H_{2 n}+\left(1-q_{n}\right) \mathbf{W}\right]+$ $\left(1-r_{n}\right) H$. Write $r_{n}=x /\left(q_{n}+\left(1-q_{n}\right) x\right)$. Then $x H_{1}+(1-x) H \prec_{W} x H_{2}+$ $(1-x) H$ by Definition $16(\mathrm{~b})$.

Case (b)-(ii). Suppose $x H_{1}+(1-x) H \prec_{W} x H_{2}+(1-x) H$. Let $\left\{H_{3 n}\right\} \Rightarrow$ $x H_{1}+(1-x) H$ and $\left\{H_{4 n}\right\} \Rightarrow x H_{2}+(1-x) H$. Assume $q_{n} H_{3 n}+\left(1-q_{n}\right) H_{n}$ $\prec *($ or $\approx *) q_{n} H_{4 n}+\left(1-q_{n}\right) \mathbf{W}$. Apply HL Axiom 2 to cancel acts in $H_{3 n}$ and $H_{4 n}$ common with $H$. Regroup the remainders to yield a $\prec *$-relation of the desired form for Definition 16(b): $q_{n}^{\prime} H_{1 n}+\left(1-q_{n}^{\prime}\right) H_{n} \prec *($ or $\approx *) q_{n}^{\prime} H_{2 n}+$ $\left(1-q_{n}^{\prime}\right) \mathbf{W}$, where $\left\{H_{1 n}\right\} \Rightarrow H_{1}$ and $\left\{H_{2 n}\right\} \Rightarrow H_{2}$. (A simple calculation shows that $\lim _{n \rightarrow \infty}\left\{q_{n}^{\prime}\right\}=q^{\prime} \geq 0.5$.) Thus $H_{1} \prec * H_{2}$.

Next, we give the details for HL Axiom 3(a) [Axiom 3(b) follows similarly.]

HL Axiom 3(a) (Archimedes). Assume $H_{n} \prec_{W} M_{n}$ and $M \prec_{W} N$, where $\left\{H_{n}\right\} \Rightarrow H$ and $\left\{M_{n}\right\} \Rightarrow M$. We are to show that $H \prec_{W} N$. Again, there are four cases to consider, depending upon how (infinitely many of) the first and the second $\prec_{W}$-preferences arise through Definition 16 . The argument is most complicated in case clause 16(b) is used throughout.

That is, assume $\exists\left\{R_{n_{m}}, S_{n} \in \mathbf{H}_{\mathbf{R}}\right\}, \exists\left\{H_{n_{m}}^{\prime}\right\}, \exists\left\{M_{n_{m}}^{\prime}\right\}, \exists\left\{M_{n}^{\prime \prime}\right\}$ and $\exists\left\{N_{n}^{\prime}\right\}$ such that, $\forall n$, as $m \rightarrow \infty,\left\{H_{n_{m}}^{\prime}\right\} \Rightarrow H_{n}$ and $\left\{M_{n_{m}}^{\prime}\right\} \Rightarrow M_{n}$, while as $n \rightarrow \infty$, 
$\left\{M_{n}^{\prime \prime}\right\} \Rightarrow M$ and $\left\{N_{n}^{\prime}\right\} \Rightarrow N$. Also assume $\exists\left\{q_{n_{m}}, s_{n} \geq 0.5\right\}$, so $\forall n, \lim _{m \rightarrow \infty}\left\{q_{n_{m}}\right\}$ $=q_{n}<1$ and $\lim _{n \rightarrow \infty}\left\{s_{n}\right\}=s<1$. By Definition 16,

$$
\begin{gathered}
q_{n_{m}} H_{n_{m}}^{\prime}+\left(1-q_{n_{m}}\right) R_{n_{m}} \prec *(\text { or } \approx *) q_{n_{m}} M_{n_{m}}^{\prime}+\left(1-q_{n_{m}}\right) \mathbf{W}, \\
s_{n} M_{n}^{\prime \prime}+\left(1-s_{n}\right) S_{n} \prec *(\text { or } \approx *) s_{n} N_{n}^{\prime}+\left(1-s_{n}\right) \mathbf{W} .
\end{gathered}
$$

Since $\left\{H_{n}\right\} \Rightarrow H$ and $\left\{M_{n}\right\} \Rightarrow M, \forall n, \exists\left(m^{*}=m(n)\right)$ so that, as $n \rightarrow \infty$, both $\left\{H_{n_{m^{*}}}^{\prime}\right\} \Rightarrow H$ and $\left\{M_{n_{m^{*}}}^{\prime}\right\} \Rightarrow M$. Moreover, we may choose (a subsequence of) these $m^{*}$ so that $\lim _{n \rightarrow \infty}\left\{q_{n_{m^{*}}}\right\}=q, 0.5 \leq q<1$. Thus we have

$$
q_{n_{m^{*}}} H_{n_{m^{*}}}^{\prime}+\left(1-q_{n_{m^{*}}}\right) R_{n_{m^{*}}} \prec *(\text { or } \approx *) q_{n_{m^{*}}} M_{n_{m^{*}}}^{\prime}+\left(1-q_{n_{m^{*}}}\right) \mathbf{W} \text {. }
$$

An application of the first two axioms to (B2) and (B3) yields

$$
\begin{aligned}
& x_{n}[\text { left side }(\mathrm{B} 2)]+\left(1-x_{n}\right)[\text { left side }(\mathrm{B} 3)] \\
& \quad \prec *\left(\text { or } \approx^{*}\right) x_{n}[\operatorname{right} \operatorname{side}(\mathrm{B} 2)]+\left(1-x_{n}\right)[\text { right side(B3)]. }
\end{aligned}
$$

Let $x_{n}=s_{n} /\left(s_{n}+q_{n_{m^{*}}}\right)$. Then as both $\left\{M_{n_{m^{*}}}^{\prime}\right\} \Rightarrow M$ and $\left\{M_{n}^{\prime \prime}\right\} \Rightarrow M$, we may cancel acts common to $M$ on both sides of (B4) to yield $z_{n} H_{n}^{\prime \prime}+\left(1-z_{n}\right) T_{n} \prec *$ (or $\left.\approx^{*}\right) z_{n} N_{n}^{\prime}+\left(1-z_{n}\right) \mathbf{W}$, where $\left\{H_{n}^{\prime \prime}\right\} \Rightarrow H,\left(N_{n}^{\prime \prime}\right) \Rightarrow N, T_{n} \in H_{\mathbf{R}}$ and $\lim _{n \rightarrow \infty}\left\{z_{n}\right\}=z=s q /(s+q-s q)$. Last, $0.5 \leq z<1$ because $0.5 \leq s, q<1$. Therefore, by Definition 16(b), $H \prec_{W} N$ as required.

Finally, Theorem 2 is concluded by repeating this construction in a dualized form: extend the preference $\prec_{W}$ to $\prec^{\prime}$ by introducing the act $\mathbf{B}$ and making it most preferred in $\mathbf{H}_{\mathbf{R} \cup\{\mathbf{W}\}}$.

C. Proof of Theorem 3. We show (by induction) how to extend $\prec\left(=\prec_{0}\right)$ while preserving Axioms 2 and 3 over simple lotteries until the desired weak order is achieved. At stage $i$ of the induction, the strategy is to identify a utility $v_{i}$ for act $\tilde{H}_{i} \in \mathscr{H}$, where $v_{i}$ is chosen (arbitrarily) from a (convex) set of target utilities for $\tilde{H}_{i}, \mathscr{T}_{i}\left(\tilde{H}_{i}\right)$. We create the partially ordered preference $\prec_{i}$ so that $\tilde{H}_{i} \approx_{i} v_{i} B+\left(1-v_{i}\right) W$.

Begin with a function $\mathscr{T}$ which provides a set of target "utilities" for all elements of $\mathbf{H}_{\mathbf{R}}$. We use $\mathbf{W}$ and $\mathbf{B}$ as the 0 and 1 of our utility. Assume $\left\{H_{n}\right\} \Rightarrow H$ and $H_{n} \in \mathbf{H}_{\mathbf{R}}$. For $i=1$, by Definitions 17 and 18, $v_{1}^{*}(H)$ is the lim inf of the quantities $x_{n}$ for which $H_{n} \prec x_{n} \mathbf{B}+\left(1-x_{n}\right) \mathbf{W}$ and $v_{1 *}(H)$ is the lim sup of the quantities $x_{n}$ for which $x_{n} \mathbf{B}+\left(1-x_{n}\right) \mathbf{W} \prec H_{n}$. The two "utility" bounds, $v_{*}(H)$ and $v^{*}(H)$, do not depend upon which sequence $\left\{H_{n}\right\} \Rightarrow H$ is used. We show this for $v_{*}(H)$. The argument for $v^{*}(H)$ is the obvious dual.

Claim 1. Let $\left\{H_{n}\right\} \Rightarrow H$ and $\left\{H_{n}^{\prime}\right\} \Rightarrow H$. Then $v_{*}(H)$ is the same for both sequences.

Proof. Suppose $v_{*}(H)=\lim \sup \left\{x_{n}: x_{n} B+\left(1-x_{n}\right) W \prec H_{n}\right\}$. Then we show that $v_{*}(H) \leq \lim \sup \left\{x_{n}: x_{n} B+\left(1-x_{n}\right) W \prec H_{n}^{\prime}\right\}$. This suffices, since by symmetry with $\left\{H_{n}^{\prime}\right\}$, when $v_{*}^{\prime}(H)=\lim \sup \left\{x_{n}: x_{n} B+\left(1-x_{n}\right) W \prec H_{n}^{\prime}\right\}$, 
then $v_{*}^{\prime}(H) \leq \lim \sup \left\{x_{n}: x_{n} B+\left(1-x_{n}\right) W \prec H_{n}\right\}$; hence, $v_{*}(H)=v_{*}^{\prime}(H)$. Since both sequences $\left\{H_{n}\right\}$ and $\left\{H_{n}^{\prime}\right\}$ converge to act $H$, we can write each pair $\left(H_{n}, H_{n}^{\prime}\right)$ as the pair $\left(y_{n} K_{n}+\left(1-y_{n}\right) M_{n}, y_{n} K_{n}+\left(1-y_{n}\right) M_{n}^{\prime}\right)$, where $\lim _{n \rightarrow \infty} y_{n}=1$. Assume all but finitely many $y_{n}<1$; else we are finished. Acts $M_{n}$ and $M_{n}^{\prime}$ belong to $\mathbf{H}_{\mathbf{R}}$ because $H_{n}$ and $H_{n}^{\prime}$ do. Of course, neither of the two sequences of acts $\left\{M_{n}\right\}$ and $\left\{M_{n}^{\prime}\right\}$ need be convergent, but $\left\{K_{n}\right\} \Rightarrow H$. For each $n$, define the act $N_{n}=y_{n} K_{n}+\left(1-y_{n}\right) \mathbf{W}$. Clearly, $\left\{N_{n}\right\} \Rightarrow H$. It follows from the preference $\mathbf{W} \prec M_{n}$ that $N_{n} \prec H_{n}$ and from the preference $\mathbf{W} \prec M_{n}^{\prime}$ that $N_{n} \prec H_{n}^{\prime}$. By hypothesis, there exists a sequence $\left\{x_{n}\right\}$ such that $x_{n} \mathbf{B}+\left(1-x_{n}\right) \mathbf{W} \prec H_{n}$ and $\lim _{n \rightarrow \infty}\left\{x_{n}\right\}=v_{*}(H)$. Let $\alpha_{n}$ be the maximum of 0 and $\left(x_{n}+y_{n}-1\right)$. Since $x_{n} \mathbf{B}+\left(1-x_{n}\right) \mathbf{W} \prec y_{n} K_{n}+\left(1-y_{n}\right) \mathbf{B}$, then $\alpha_{n} \mathbf{B}$ $+\left(1-\alpha_{n}\right) \mathbf{W} \prec y_{n} K_{n}+\left(1-y_{n}\right) \mathbf{W}=N_{n}$. Transitivity of $\prec$ yields $\alpha_{n} \mathbf{B}+$ $\left(1-\alpha_{n}\right) \mathbf{W} \prec H_{n}^{\prime}$. However, as $\lim _{n \rightarrow \infty}\left\{y_{n}\right\}=1$ and $\lim _{n \rightarrow \infty}\left\{x_{n}\right\}=v_{*}(H)$, then $\lim _{n \rightarrow \infty}\left\{\alpha_{n}\right\}=v_{*}(H)$. Thus, $v_{*}(H) \leq \lim \sup \left\{x_{n}: x_{n} \mathbf{B}+\left(1-x_{n}\right) \mathbf{W} \prec H_{n}^{\prime}\right\}$.

Observe that if $v^{*}\left(H_{1}\right)<v_{*}\left(H_{2}\right)$, then $H_{1} \prec H_{2}$, by Axiom 3 and the fact that $\mathbf{W} \prec \mathbf{B}$. However, these "utility" bounds are merely sufficient, not necessary, for the $\prec$-preference $H_{1} \prec H_{2}$.

Proof of Lemma 3.1. Note that $x B+(1-x) W \prec y B+(1-y) W$ whenever $x<y$.

(i) Suppose, on the contrary, that $v^{*}(H)<v_{*}(H)$. Then by Corollary 2.5 applied twice over, $v^{*}(H) B+\left(1-v^{*}(H)\right) W \approx H \approx v_{*}(H) B+(1-$ $\left.v_{*}(H)\right) W$. Since $v^{*}(H)<v_{*}(H)$, also $v^{*}(H) B+\left(1-v^{*}(H)\right) W \prec v_{*}(H) B+$ $\left(1-v_{*}(H)\right) W$, contradicting the $\approx$-relation between them, as just derived.

(ii) This is immediate, by similar reasoning.

Next, we show that $\prec$ may be extended to $\prec_{H}$, a strict partial order satisfying HL Axioms 2 and 3 , in which $H \approx_{H} v_{H} B+\left(1-v_{H}\right) W$ and where the utility $v_{H}$ may be any value in the interior of the closed target set $\mathscr{T}(H)$. We resolve when an endpoint of the (closed) target set may be a utility afterward.

Definition 20. For $H \in \mathbf{H}_{\mathbf{R}}$, let $v \in \operatorname{int} \mathscr{T}(H)$. [When $\mathscr{T}(H)$ has no interior, when $v_{*}(H)=v^{*}(H)=v$, then by Lemma 3.1(ii), $H \approx v \mathbf{B}+(1-$ $v)$ W. Thus it is appropriate that Definition 20 creates no extension of $\prec$. Then act $H$ already has its "utility" fixed by $\prec$.] Define $\prec_{H}$ by

$$
\begin{gathered}
\left(H_{1} \prec_{H} H_{2}\right) \quad \text { iff } \exists(0<x<1) \exists\left(G, G^{\prime}\right), \\
x H_{1}+(1-x) G \prec x H_{2}+(1-x) G^{\prime},
\end{gathered}
$$

where $G$ and $G^{\prime}$ are symmetric mixtures of $H$ and $v B+(1-v) W$.

Specifically, $\exists y$ with $G=y H+(1-y)[v B+(1-v) W]$ and $G^{\prime}=y[v B+$ $(1-v) W]+(1-y) H$. 
LEMMA 3.2. $\prec_{H}$ extends $\prec$.

Proof. Assume $H_{1} \prec H_{2}$. Choose $y=0.5$ in Definition 20 , so $G=G^{\prime}$. By Axiom $2, x H_{1}+(1-x) G \prec x H_{2}+(1-x) G^{\prime}$, so that $\left(H_{1} \prec_{H} H_{2}\right)$.

Lemma 3.3. $\succ_{H}$ satisfies HL Axioms 1-3.

Proof. We establish Axioms 1-3 separately.

$H L$ Axiom 1 (irreflexivity). Assume not $\left(H_{1} \prec_{H} H_{1}\right)$. Then $x H_{1}+(1-x) G$ $\prec x H_{1}+(1-x) G^{\prime}$, which by Axiom 2 yields $G \prec G^{\prime}$. By Definition 20 and another application of Axiom 2, either $H \prec v B+(1-v) W$ or else $v B+$ $(1-v) W \prec H$. Either contradicts the relation $H \sim v B+(1-v) W$. That follows from the assumption $v_{*}<v<v^{*}$.

HL Axiom 1 (transitivity). Assume $\left(H_{1} \prec_{H} H_{2}\right)$ and $\left(H_{2} \prec_{H} H_{3}\right)$. Then we have

$$
\begin{aligned}
x H_{1}+(1-x) G & \prec x H_{2}+(1-x) G^{\prime} \text { and } \\
w H_{2}+(1-w) J & \prec w H_{3}+(1-w) J^{\prime},
\end{aligned}
$$

where both pairs $\left(G, G^{\prime}\right)$ and $\left(J, J^{\prime}\right)$ satisfy Definition 20 . These equations may be combined to create $\forall z, z\left(x H_{1}+(1-x) G\right)+(1-z)\left(w H_{2}+\right.$ $(1-w) J) \prec z\left(x H_{2}+(1-x) G^{\prime}\right)+(1-z)\left(w H_{3}+(1-w) J^{\prime}\right)$. Choose $z /(1$ $-z)=w / x$. By HL Axiom 2, we may cancel the common term $z x H_{2}[=(1-$ $z) w H_{2}$ ] from both sides and recombine the pairs $(G, J)$ and $\left(G^{\prime}, J^{\prime}\right)$ to yield $u H_{1}+(1-u) K \prec u H_{3}+(1-u) K^{\prime}$. Thus, $H_{1} \prec_{H} H_{3}$.

$H L$ Axiom 2. Argue that $H_{1} \prec_{H} H_{2}$ iff $y H_{1}+(1-y) G \prec y H_{2}+(1-y) G^{\prime}$ iff $\forall(0<z<1), \quad z\left(y H_{1}+(1-y) G\right)+(1-z) H_{3} \prec z\left(y H_{2}+(1-y) G^{\prime}\right)+$ $(1-z) H_{3}$ iff $(\exists w) w\left(x H_{1}+(1-x) H_{3}\right)+(1-w) G \prec w\left(x H_{2}+(1-x) H_{3}\right)$ $+(1-w) G^{\prime}$ (choose $\left.w=z y / x\right)$ iff $x H_{1}+(1-x) H_{3} \prec_{H} x H_{2}+(1-x) H_{3}$.

$H L$ Axiom 3(a). Assume $\forall n\left(M_{n} \prec_{H} N_{n}\right)$, and $\left(N \prec_{H} O\right)$. Then show $\left(M \prec_{H} O\right)$.

1. Thus (a) $\left(x_{n} M_{n}+\left(1-x_{n}\right) G_{n}\right) \prec\left(x_{n} N_{n}+\left(1-x_{n}\right) G_{n}^{\prime}\right)$ and also (b) $(y N+$ $(1-y) J) \prec\left(y O+(1-y) J^{\prime}\right)$.

As Definition 20 applies to the pairs $\left(G_{n}, G_{n}^{\prime}\right),\left(J, J^{\prime}\right)$, we may cancel (by Axiom 2) common terms to create:

2. Either (a) $u_{n} M_{n}+\left(1-u_{n}\right) H \prec u_{n} N_{n}+\left(1-u_{n}\right)(v B+(1-v) W)$ or (b) $u_{n} M_{n}+\left(1-u_{n}\right)(v B+(1-v) W) \prec u_{n} N_{n}+\left(1-u_{n}\right) H$.

3. Also, in addition, either (a) $w N+(1-w) H \prec w O+(1-w)(v B+$ $(1-v) W)$ or $(\mathrm{b}) w N+(1-w)(v B+(1-v) W) \prec w O+(1-w) H$, where $u_{n} \geq x_{n}$ and $w \geq y$.

At least one of 2(a) or 2(b) occurs infinitely often. Without loss of generality, assume 2(a) does. Since $v_{*}(H)<v<v^{*}(H)$, then $\liminf \left\{u_{n}\right\}=u>0$, in this infinite subsequence. [Only here do we use the fact that $v$ is an interior point of $\mathscr{T}(H)$. See Lemma 3.5 for additional remarks.] 
Thus, we are justified in considering a convergent sequence of the form 2(a), also indexed by $n$, with coefficients converging to $u>0$. We argue by cases: Assume 3(a) obtains. Using Axiom 2, we mix in the act $H$ to both sides of 2(a) and the act $(v B+(1-v) W)$ to both sides of 3(a), yielding:

$$
\text { 4(a) } \begin{aligned}
& x\left[u_{n} M_{n}+\left(1-u_{n}\right) H\right]+(1-x) H \quad \prec \quad x\left[u_{n} N_{n}+\left(1-u_{n}\right)(v B+(1-v) W)\right] \\
& \quad+(1-x) H . \\
& \text { 4(b) } z[w N+(1-w) H]+(1-z)(v B+(1-v) W) \prec z[w O+(1-w) \\
&\times(v B+(1-v) W)]+(1-z)(v B+(1-v) W) .
\end{aligned}
$$

Choose $x u=z w=q \neq 0,(1-x)=z(1-w)$. Note all of the following occur: the l.h.s. of 4(a) converges to the act $(q M+(1-q) H)$; the r.h.s. of $4(\mathrm{~b})$ is the act $(q O+(1-q)(v B+(1-v) W))$; the r.h.s. of $4(\mathrm{a})$ converges to the l.h.s. of 4(b). Then by HL Axiom 3(a), $(q M+(1-q) H) \prec(q O+(1-q)(v B+$ $(1-v) W)$ ), so by Definition $20, M \prec_{H} O$.

In case $3(\mathrm{~b})$ obtains, instead, we modify this argument by mixing the term $(v B+(1-v) W)$ into $2(\mathrm{a})$ in case $u<w$ or into $3(\mathrm{~b})$ in case $w<u$, so that (as above) the r.h.s. of 4(a) converges to the l.h.s. of 4(b) and so forth.

HL Axiom 3(b). This is verified just as HL Axiom 3(a) is.

Thus, $\prec_{H}$ satisfies the axioms.

To complete our discussion of $\mathscr{T}(H)$, we explain when $\prec_{H}$ may be created using an endpoint of the target set. To motivate our analysis, consider when a partial order $\prec$ precludes an extension by a particular new preference or indifference.

DEFINITION 21. Say that a preference for act $H_{a}$ over act $H_{b}$ is precluded by the partial order $\prec$, denoted as $\neg\left(H_{b} \prec H_{a}\right)$, if there exist two convergent sequences of acts $\left\{H_{a, n}\right\} \Rightarrow H_{a}$ and $\left\{H_{b, n}\right\} \Rightarrow H_{b}$, where $(\forall n) H_{a, n} \prec H_{b, n}$. [ Note: $H_{a} \approx H_{b}$ or $H_{a} \prec H_{b}$ implies the condition $\neg\left(H_{b} \prec H_{a}\right)$.]

Definition 22. Say that indifference between acts $H_{a}$ and $H_{b}$ is precluded by the partial order $\prec$, denoted as $\neg\left(H_{b} \approx H_{a}\right)$, if assuming the relation $\left(H_{a} \approx H_{b}\right)$, the three axioms and the preferences $\prec$ all yield a preference precluded by $\prec$.

EXAMPLE 3.2. We illustrate $\neg\left(H_{b} \approx H_{a}\right)$. Suppose $\prec$ satisfies the axioms and the following obtain. Let $H_{a} \sim H_{b}$. However, there exist two convergent sequences of acts $\left\{M_{n}\right\} \Rightarrow M$ and $\left\{N_{n}\right\} \Rightarrow N$ and coefficients $\left\{x_{n}: x_{n}>0\right.$, $\left.\lim _{n \rightarrow \infty} x_{n}=0\right\}$, where $x_{n} M_{n}+\left(1-x_{n}\right) H_{a} \prec x_{n} N_{n}+\left(1-x_{n}\right) H_{b}$. However, for some $y>0, \quad y N+(1-y) H_{a} \prec y M+(1-y) H_{b}$. Thus, $\left(H_{a} \approx H_{b}\right.$ ) entails $M_{n} \prec N_{n}$ and $N \prec M$. By Axiom 3, then $M \prec M$, which is a $\prec$-precluded preference since $M \approx M$ obtains whenever $\prec$ satisfies the axioms.

[We sketch a model for these $\prec$-preferences. Let $H_{a}=v_{*} B+\left(1-v_{*}\right) W$ and $H_{b}=H$. Suppose $\mathscr{V}$ is a set of utilities $\left\{V_{d}: 1>d>0 ; V_{d}(H)=v_{*}+d\right.$ and $V_{d}(M)-V_{d}(N)=d^{0.5}$. Consider $\prec_{\mathscr{V}}$, when $\mathscr{T}(H)=\left[v_{*}, v^{*}\right]$ yet $H_{a} \prec_{\mathscr{V}}$ 
$H_{b}$. Let $\prec$ be as $\prec_{\mathscr{V}}$ except that $H_{a} \sim H_{b}$ is forced. $V_{d}\left(x M+(1-x) v_{*}\right) \leq$ $V_{d}(x N+(1-x) H)$ entails that $x /(1-x) \leq d^{0.5}$. Since $d$ assumes each value in (0.1), $x=0$ is a necessary condition for the preferences of Example 3.2.]

ClaIm 2. If both $\neg\left(H_{a} \prec H_{b}\right)$ and $\neg\left(H_{b} \prec H_{a}\right)$, then $H_{a} \approx H_{b}$.

Proof. When $\neg\left(H_{a} \prec H_{b}\right)$ and $\neg\left(H_{b} \prec H_{a}\right)$, then there exist pairs of convergent acts $\left\{H_{a, n}\right\},\left\{H_{a, n}^{\prime}\right\} \Rightarrow H_{a}$ and $\left\{H_{b, n}\right\},\left\{H_{b, n}^{\prime}\right\} \Rightarrow H_{b}$, where $(\forall n)$ $H_{a, n} \prec H_{b, n}$ and $H_{b, n}^{\prime} \prec H_{a, n}^{\prime}$. Then by Corollary $2.5, H_{a} \approx H_{b}$.

Example 3.2 illustrates that our axioms are not strong enough to ensure the preference $H_{a} \prec H_{b}$ when, for example $\neg\left(H_{b} \prec H_{a}\right)$ and $\neg\left(H_{a} \approx H_{b}\right)$. It so happens that when both the conditions $\neg\left(H_{b} \prec H_{a}\right)$ and $\neg\left(H_{a} \approx H_{b}\right)$ obtain and these two acts do not involve the distinguished rewards $W$ and $B$, then each extension $\prec *$ of $\prec$ which fixes "utilities" for $H_{a}$ and $H_{b}$ (and where $\prec *$ arises by iteration of Definition 20) has the desired relation $H_{a} \prec *$ $H_{b}$. Our specific problem, however, is with the case when one of these two acts is a utility endpoint of the (closed) target set $\mathscr{T}(H)$, for example, let $H_{a}=v_{*} B+\left(1-v_{*}\right) \mathbf{W}$ and $H_{b}=H$, as in the model for the $\prec$-preferences sketched in Example 3.2. We require an extra consideration, then, to determine whether, though $v_{*} B+\left(1-v_{*}\right) W \sim H$, a combination of $\prec-$ preferences arises which prohibits an extension $\prec_{H}$ of $\prec$ that assigns the "utility" $v_{*}$ for $H$.

Our solution is to show how to extend the partial order $\prec$ to a partial order $\prec^{+}$that includes all the so-called missing preferences $H_{a} \prec H_{b}$.

Definition 23. Define $\prec^{+}$from $\prec$ by $H_{a} \prec^{+} H_{b}$ iff $H_{a} \prec H_{b}$ or, both $\neg\left(H_{b} \prec H_{a}\right)$ and $\neg\left(H_{a} \approx H_{b}\right)$.

The next lemma establishes (very weak) conditions under which the $\prec^{+}$-closure of a partial order $\prec$ satisfies all three axioms. In particular, it is not necessary that $\prec$ satisfies HL Axiom 3. [The condition $\neg\left(H_{b} \prec H_{a}\right)$ is well defined according to Definition 23 even though $\prec$ is known only to satisfy HL Axioms 1 and 2 . Specifically, the indifference relation $\approx$ is well defined and satisfies all those properties, e.g., Corollary 2.4, which depend on HL Axioms 1 and 2 alone.]

LEMMA 3.4. The partial order $\prec^{+}$satisfies all three axioms provided $\prec$ satisfies the first two axioms, HL Axioms 1 and 2, and provided closure of $\prec$ under all three axioms does not produce a $\prec$-precluded preference.

Proof. We verify the axioms separately.

$H L$ Axiom 1 (irreflexivity). Since $H \approx H$ obtains and $\prec$ yields no $\prec-$ precluded preference (under the three axioms), no act $H$ satisfies $H \prec^{+} H$. That is, $H \approx H$ is not $\prec$-precluded. 
HL Axiom 1 (transitivity). Assume $H_{a} \prec^{+} H_{b}$ and $H_{b} \prec^{+} H_{c}$. Each of these $\prec^{+}$-preferences may arise two ways, according to Definition 23 . We examine a general case: $\neg\left(H_{b} \prec H_{a}\right), \neg\left(H_{c} \prec H_{b}\right), \neg\left(H_{a} \approx H_{b}\right)$ and $\neg\left(H_{b} \approx H_{c}\right)$. We show that (i) $\neg\left(H_{c} \prec H_{a}\right)$ and (ii) $\neg\left(H_{a} \approx H_{c}\right)$.

(i) From the two assumptions $\neg\left(H_{b} \prec H_{a}\right)$ and $\neg\left(H_{c} \prec H_{b}\right)$, we conclude that there exist convergent sequences $\left\{H_{a, n}\right\} \Rightarrow H_{a},\left\{H_{b, n}\right\}$ and $\left\{H_{b, n}^{\prime}\right\} \Rightarrow H_{b}$ and $\left\{H_{c, n}\right\} \Rightarrow H_{c}$, with $(\forall n) H_{a, n} \prec H_{b, n}$ and $H_{b, n}^{\prime} \prec H_{c, n}$. Thus, by Axioms 1 and $2,0.5 H_{a, n}+0.5 H_{b, n}^{\prime} \prec 0.5 H_{b, n}+0.5 H_{c, n}$. Using HL Axiom 2 to cancel common terms in $H_{b, n}$ and $H_{b, n}^{\prime}$, we obtain $\prec$-preferences of the form $H_{a, n}^{\prime} \prec H_{c, n}^{\prime}$, where $\left\{H_{a, n}^{\prime}\right\} \Rightarrow H_{a}$ and $\left\{H_{c, n}^{\prime}\right\} \Rightarrow H_{c}$. Thus, $\neg\left(H_{c} \prec H_{a}\right)$.

(ii) Assume $H_{a} \approx H_{c}$. Because $H_{a, n} \prec H_{b, n}$ we may construct new convergent sequences $\left\{H_{c, n}^{\prime}\right\} \Rightarrow H_{c}$ and $\left\{H_{b, n}^{\prime \prime}\right\} \Rightarrow H_{b}$, where $H_{c, n}^{\prime} \prec H_{b, n}^{\prime \prime}$.

This exercise is done as follows. From the indifference $H_{a} \approx H_{c}$ conclude $(1 / n) W+([n-1] / n) H_{c} \prec(1 / n) B+([n-1] / n) H_{a}$. Then $0.5 H_{a, n}+$ $0.5\left[(1 / n) W+([n-1] / n) H_{c}\right] \prec 0.5 H_{b, n}+0.5\left[(1 / n) B+([n-1] / n) H_{a}\right]$. Use Axiom 2 to cancel common terms involving act $H_{a}$.

We already have assumed $H_{b, n}^{\prime} \prec H_{c, n}$. Then, since we are entitled to use HL Axiom 3 in determining the consequences of adopting $H_{a} \approx H_{c}$, by Corollary 2.5, from $H_{a} \approx H_{c}$ we derive $H_{b} \approx H_{c}$. ᄀ $\left(H_{b} \approx H_{c}\right)$ means that adding the $\approx$-indifference $H_{b} \approx H_{c}$ yields a $\prec$-precluded preference. Thus, adding $H_{a} \approx H_{c}$ to $\prec$ yields the same $\prec$-precluded preference. Hence, $\neg\left(H_{a} \approx H_{c}\right)$.

HL Axiom 2 (independence). This axiom is easy to verify, since $\prec$ satisfies HL Axioms 1 and 2. We illustrate the argument from right to left. Suppose $x H_{a}+(1-x) H \prec^{+} x H_{b}+(1-x) H$. We are to show that (i) $\neg\left(H_{b} \prec H_{a}\right)$ and (ii) $\neg\left(H_{a} \approx H_{b}\right)$.

(i) We know that both $\neg\left(x H_{b}+(1-x) H \prec x H_{a}+(1-x) H\right)$ and $\neg\left(x H_{a}+(1-x) H \approx x H_{b}+(1-x) H\right)$. As in previous cases, we may assume existence of convergent sequences $\left.\left\{H_{1, n}\right\} \Rightarrow x H_{a}+(1-x) H\right)$ and $\left\{H_{2, n}\right\}$ $\left.\Rightarrow x H_{b}+(1-x) H\right)$, where $H_{1, n} \prec H_{2, n}$. Use HL Axiom 2 to cancel common terms (involving act $H$ ) in each pair $H_{1, n}$ and $H_{2, n}$. The results are $\prec-$ preferences of the form $H_{a, n} \prec H_{b, n}$, with $\left\{H_{a, n}\right\} \stackrel{\Rightarrow}{\Rightarrow} H_{a}$ and $\left\{H_{b, n}\right\} \Rightarrow H_{b}$. Thus, $\neg\left(H_{b} \prec H_{a}\right)$.

(ii) By Corollary 2.4, from the assumption $H_{a} \approx H_{b}$ it follows that $x H_{a}+$ $(1-x) H \approx x H_{b}+(1-x) H$, which yields a $\prec$-precluded preference as $\neg\left(x H_{a}+(1-x) H \approx x H_{b}+(1-x) H\right)$.

$H L$ Axiom 3(a). Assume $M_{n} \prec^{+} N_{n}$ and $N \prec{ }^{+} O$, where $\left\{M_{n}\right\} \Rightarrow M$ and $\left\{N_{n}\right\} \Rightarrow N$. We need to show that (a) $\neg(O \prec M)$ and (b) $\neg(M \approx O)$.

(a) Thus $\neg\left(N_{n} \prec M_{n}\right), \neg\left(M_{n} \approx N_{n}\right), \neg(O \prec N)$ and $\neg(N \approx O)$. As in previous cases, assume each of these $\prec$-precluded preferences arises from corresponding sequences of $\prec$-preferences. That is, for each $n$ there is a pair of convergent sequences $\lim _{j \rightarrow \infty}\left\{M_{n, j}\right\} \Rightarrow M_{n}$ and $\left\{N_{n, j}\right\} \Rightarrow N_{n}$, where $M_{n, j} \prec$ $N_{n, j}$. Also, there is a pair of convergent sequences $\left\{N_{n}^{\prime}\right\} \Rightarrow N$ and $\left\{O_{n}\right\} \stackrel{\Rightarrow}{\Rightarrow}$, where $N_{n}^{\prime} \prec O_{n}$. Since $\left\{M_{n}\right\} \Rightarrow M$ and $\left\{N_{n}\right\} \Rightarrow N$, for each $n$ we may choose a value $j_{n}$ so that $\lim _{n \rightarrow \infty}\left\{M_{n, j_{n}}\right\} \Rightarrow M$ and $\left\{N_{n, j_{n}}\right\} \Rightarrow N$. Of course, $M_{n, j_{n}} \prec N_{n, j_{n}}$. 
Then, $0.5 M_{n, j_{n}}+0.5 N_{n}^{\prime} \prec 0.5 N_{n, j_{n}}+0.5 O_{n}$. Use HL Axiom 2 to cancel terms common to act $N$, yielding $\prec$-preferences sufficient for $\neg(O \prec M)$.

(b) If we assume $M \approx O$, then (because $M_{n, j_{n}} \prec N_{n, j_{n}}$ ) there are sequences $\left\{O_{n}^{\prime}\right\} \Rightarrow O$ and $\left\{N_{n}^{\prime \prime}\right\} \Rightarrow N$, with $O_{n}^{\prime} \prec N_{n}^{\prime \prime}$. Since $N_{n}^{\prime} \prec O_{n}$, using Axiom 3, by Corollary 2.5 , then $N \approx O$. However, $\neg(N \approx O)$. Hence, assuming $M \approx O$ entails some $\prec$-precluded preference. Therefore, $\neg(M \approx O)$. HL Axiom $3(\mathrm{~b})$ is demonstrated in the identical fashion.

In the next definition, based on Lemma 3.4, we indicate whether either endpoint of $\mathscr{T}(H)$ is eligible as a utility for $H$ when extending $\prec$ to form $\prec_{H}$.

Definition 24. Say that $v_{*}(H)$ is a candidate utility for $H$ if $v_{*} \mathbf{B}+$ $\left(1-v_{*}\right) \mathbf{W} \sim^{+} H$. Likewise, $v^{*}(H)$ is a candidate utility for $H$ if $H^{+}$ $v^{*} \mathbf{B}+\left(1-v^{*}\right) \mathbf{W}$.

We conclude our discussion of the extension $\prec_{H}$ for the special case when it is generated by a target set endpoint provided, of course, the endpoint is a candidate utility for $H$. The idea behind the extension, is that as it stands, Definition 20 fails with $v=v_{*}$ or $v=v^{*}$ only because the resulting partial order is incomplete with respect to Axiom 3. (See Lemma 3.5, below.) Then, in light of Lemma 3.4, the +-closure (using Definition 23) corrects the omissions. (See Lemma 3.6.)

When extending $\prec$ with a candidate utility, $v=v_{*}$ or $v=v^{*}$, that is, using an endpoint of $\mathscr{T}(H)$, we define the extension $\prec_{H}$ in two steps, as follows: Analogous with Definition 20, let $G$ and $G^{\prime}$ be symmetric mixtures of $H$ and $v B+(1-v) W$.

Definition 25. Define $H_{1} \prec_{\#} H_{2}$ iff $\exists(0<x<1) \exists\left(G, G^{\prime}\right), x H_{1}+$ $(1-x) G \prec x H_{2}+(1-x) G^{\prime}$, and let $\prec_{H}$ result by closing $\prec_{\#}$ using Definition 23, that is, $\prec_{H}=\prec_{\#}^{+}$.

LEMMA 3.5. The partial order $\prec_{\#}$ extends $\prec$ and satisfies axioms $H L$ Axioms 1 and 2.

Proof. Since $v$ is a candidate utility, $v \mathbf{B}+(1-v) \mathbf{W} \sim H$. Then, as Definition 25 duplicates Definition 20, the proofs from Lemmas 3.2 and 3.3 apply to show that $\prec_{\#}$ extends $\prec$ and satisfies the first two axioms.

Lemma 3.6. The partial order $\prec_{\#}^{+}$extends $\prec$ and satisfies all three axioms.

Proof. In light of Lemma 3.5, the result follows by Lemma 3.4 once we show that $\prec_{\#}$ may be closed under the axioms without generating $\mathrm{a} \prec_{\#}{ }^{-}$ precluded preference. Note that $\prec_{\#}$ extends $\prec$ by some, but not necessarily 
all, preferences entailed (by the three axioms) from the $\approx$-indifference $H \approx v B+(1-v) W$. Then, since $v$ is a candidate utility, closing $\prec_{\#}$ under the three axioms does not lead to a $\prec$-precluded preference. We claim, next, that it does not lead to a $\prec_{\#}$-precluded preference either. Suppose, on the contrary, it does. Suppose, for example, closing $\prec_{\#}$ with the axioms results in a relation $H_{b} \prec_{\#} H_{a}$, where also $\neg\left(H_{b} \prec_{\#} H_{a}\right)$. The former means that adding $H \approx v B+(1-v) W$ to the set of $\prec$-preferences entails (by the axioms) that $H_{b} \prec H_{a}$. The latter requires that, for two convergent sequences $\left\{H_{a, n}\right\}$ and $\left\{H_{b, n}\right\},\left(H_{a, n} \prec_{\#} H_{b, n}\right)$. Thus, adding $H \approx v B+(1-v) W$ to the set of $\prec$-preferences entails (by the axioms) that $\left(H_{a, n} \prec H_{b, n}\right)$. By HL Axiom 3, these lead to a $\prec$-precluded preference $\left(H_{b} \prec H_{b}\right)$. Then $v$ is not a candidate utility for $H$ with respect to $\prec$, a contradiction.

Thus, with Definition 20, we have indicated how to extend $\prec$ to $\prec_{H}$, where act $H$ is assigned a utility $v$ from the interior of its target set $\mathscr{T}(H)$, and with Definition 25, how to extend to $\prec_{H}$ using a candidate utility endpoint.

We interject two simple, but useful results about $\approx_{H}$-indifferences. The first confirms that the extension $\prec_{H}$ preserves $\approx$-indifferences. The second shows that the extension $\prec_{H}$ makes act $H \approx_{H}$-indifferent with its assigned utility $v$.

\section{LEMmA 3.7. If $M \approx N$, then $M \approx_{H} N$.}

Proof. Suppose $M \approx N$ and that $x M+(1-x) H_{3} \prec_{H} H_{4}$. We are to show that $x N+(1-x) H_{3} \prec_{H} H_{4}$. By Definition 23, $\left[y\left(x M+(1-x) H_{3}\right)+\right.$ $(1-y) G] \prec\left[y H_{4}+(1-y) G^{\prime}\right]$. After rearranging terms, by Corollary 23, $\left[y\left(x N+(1-x) H_{3}\right)+(1-y) G\right] \prec\left[y H_{4}+(1-y) G^{\prime}\right]$, so that $x N+$ $(1-x) H_{3} \prec_{H} H_{4}$.

LEMma 3.8. $H \approx_{H} v \mathbf{B}+(1-v) \mathbf{W}$.

Proof. Since $\mathbf{W} \prec \mathbf{B}$, we have the following:

$$
\begin{aligned}
& (1 / n) W+[(n-1) / n][0.5 H+0.5(v B+(1-v) W)] \\
& \quad \prec(1 / n) B+[(n-1) / n][0.5 H+0.5(v B+(1-v) W)] .
\end{aligned}
$$

This equation may be written as $x_{n} H_{n}+\left(1-x_{n}\right)(v B+(1-v) W) \prec$ $x_{n} M_{n}+\left(1-x_{n}\right) H$, where $\left\{x_{n}\right\} \rightarrow 0.5, \quad\left\{H_{n}\right\} \Rightarrow H$ and $\left\{M_{n}\right\} \Rightarrow(v B+$ $(1-v) W)$. By Definition 20, $H_{n} \prec_{H} M_{n}$. Similarly, it may be written $x_{n} M_{n}^{\prime}+$ $\left(1-x_{n}\right)(H) \prec x_{n} H_{n}^{\prime}+\left(1-x_{n}\right)(v B+(1-v) W)$, where $\left\{x_{n}\right\} \rightarrow 0.5,\left\{H_{n}^{\prime}\right\} \Rightarrow H$ and $\left\{M_{n}^{\prime}\right\} \Rightarrow(v B+(1-v) W)$. By Definition 20, $M_{n}^{\prime} \prec_{H} H_{n}^{\prime}$. Then, by Corollary $2.5, H \approx_{H} v \mathbf{B}+(1-v) \mathbf{W}$.

We iterate Definition 20 (or Definition 25) in a denumerable sequence of extensions of $\prec$. 
Definition 26. Define the set $\mathscr{H}=\left\{\tilde{H}_{i}^{k}: \tilde{H}_{i}^{k}\left(s_{j}\right)=r_{1}\right.$ if $j \neq k$, and $\tilde{H}_{i}^{k}\left(s_{k}\right)=r_{i}$. Let $\mathbf{r}_{1}$ denote the constant act that yields reward $r_{1}$ in each state, so that $\mathbf{r}_{1} \in \mathscr{H}$.

LeMma 3.9. $\mathscr{H}$ is countable and finite if $\mathbf{R}$ is finite.

The proof is obvious.

[ $\mathscr{H}$ remains countable even when $\pi$ is a denumerable partition. Then it follows from HL Axiom 3 that personal probabilities over $\pi$ are $\sigma$-additive. That is, HL Axiom 3 entails "continuity": $\lim _{n \rightarrow \infty} p\left\{\cap E_{n}\right\}=p\left\{\lim _{n \rightarrow \infty} \cap E_{n}\right\}$. In the light of Fishburn's (1979), page 139, result Theorem 10.5, we conjecture that our central theorems, e.g., Theorems 3 and 6, carry over to countably infinite partitions. However, this is not evident, e.g., our proof of Claim 1 (for Theorem 3) does not apply when $\pi$ is infinite. Our use of finite partitions avoids mandating $\sigma$-additivity of personal probability.]

Hereafter, we enumerate $\mathscr{H}$ with a single subscript $i$. At stage $i$ of the induction, $\prec_{i}$ is obtained by choosing a target utility $v_{i}$ for act $\tilde{H}_{i} \in \mathscr{H}$, denoted $V\left(\tilde{H}_{i}\right)=v_{i}$. Here $v_{i} \in \mathscr{T}_{i}\left(\tilde{H}_{i}\right)$ and $\mathscr{T}_{i}(\cdot)$ identifies sets of target utilities, based on $\prec_{i-1}$. By Lemma 3.7, extensions preserve utilities already assigned, so that all utilities fixed by stage $i$ are well defined over stages $j \geq i$. Next, we show that each simple act has its "utility" $V$ determined by a finite subset of $\mathscr{H}$.

LEMMA 3.10. If $H \in \mathbf{H}_{\mathbf{R}}$ is a simple act, then there is a (finite) stage $\prec_{m}$ such that $\mathscr{T}_{m}(H)$ is a unit set, that is, by stage $\prec_{m}, H$ is assigned a precise utility $V(H)$.

Proof. First we verify that $V$ has the expected utility property over elements of $\mathscr{H}$. Consider $\tilde{H}_{a}, \tilde{H}_{b} \in \mathscr{H}$. Without loss of generality, let $b=$ $\max \{a, b\}$. Both $\tilde{H}_{a}$ and $\tilde{H}_{b}$ have their respective utilities by stage $\prec_{b}$. That is, $\tilde{H}_{a} \approx_{b} v_{a} \mathbf{B}+\left(1-v_{a}\right) \mathbf{W}$ and $\tilde{H}_{b} \approx_{b} v_{b} \mathbf{B}+\left(1-v_{b}\right) \mathbf{W}$. By Corollary 2.4, $x \tilde{H}_{a}+(1-x) \tilde{H}_{b} \approx_{b} x\left(v_{a} \mathbf{B}+\left(1-v_{a}\right) \mathbf{W}\right)+(1-x)\left(v_{b} \mathbf{B}+\left(1-v_{b}\right) \mathbf{W}\right)$. Hence, $V\left(x \tilde{H}_{a}+(1-x) \tilde{H}_{b}\right)=x V\left(\tilde{H}_{a}\right)+(1-x) V\left(\tilde{H}_{b}\right)$.

Next, write $H\left(s_{j}\right)=\sum_{i=1}^{k_{j}} P_{j}\left(r_{i}\right)$. Define the act $H_{j}^{\prime}(s)=H(s)$ if $s=s_{j}$; otherwise $H^{\prime}(s)=r_{1}$. Since $H$ is simple, each $H_{j}^{\prime}$ is a finite combination of $\tilde{H}_{i} \in \mathscr{H}$. Specifically, $H_{j}^{\prime}=\sum_{i=1}^{k_{j}} P_{j}\left(\tilde{H}_{i}^{j}\right)$, where $\tilde{H}_{i}^{j}(s)=r_{i}$ if $s=s_{j}$ and $\tilde{H}_{i}^{j}(s)=r_{1}$ otherwise. Observe that $(1 / n) H+(n-1 / n) \mathbf{r}_{1}=\Sigma(1 / n) H_{j}^{\prime}$. Thus the utility $V(H)$ is determined once $V\left(\mathbf{r}_{1}\right)$ and the $n$ values $V\left(H_{j}^{\prime}\right)$ are fixed, all of which occurs after finitely many elements of $\mathscr{H}$ are assigned their utilities.

We create a weak order $\precsim_{\omega}$ from the partial orders $\prec_{i}(i=1, \ldots)$ using the fact that each $H \in \mathbf{H}_{\mathbf{R}}$ is a limit point of simple horse lotteries. For $H \in \mathbf{H}_{\mathbf{R}}$ consider a sequence $\left\{H_{n}\right\} \Rightarrow H$, where $H_{n}$ is a simple act. Let $V(H)=\lim _{n \rightarrow \infty} V\left(H_{n}\right)$. Then: 
LEMMA 3.11. $V(H)$ is well defined.

Proof. We show that if $\left\{H_{n}\right\} \Rightarrow H$, then $\lim _{n \rightarrow \infty} V\left(H_{n}\right)$ exists and is unique. Assume $\left\{H_{n}\right\} \Rightarrow H$ and $\left\{H_{n}^{\prime}\right\} \Rightarrow H$, where all these acts belong to $\mathbf{H}_{\mathbf{R}}$. Without loss of generality, since the simple acts form a dense subset of $\mathbf{H}_{\mathbf{R}}$ under the topology of pointwise convergence, suppose that each of $H_{n}, H_{n}^{\prime}$ is simple. Then write $H_{n}$ as $y_{n} K_{n}+\left(1-y_{n}\right) M_{n}$ and $H_{n}^{\prime}$ as $y_{n} K_{n}+\left(1-y_{n}\right) M_{n}^{\prime}$, where $\lim _{n \rightarrow \infty} y_{n}=1$ and each of $K_{n}, M_{n}$ and $M_{n}^{\prime}$ is a simple act in $\mathbf{H}_{\mathbf{R}}$. By Lemma 3.10, $V\left(H_{n}\right)-V\left(H_{n}^{\prime}\right)=\left(1-y_{n}\right)\left[V\left(M_{n}\right)-V\left(M_{n}^{\prime}\right)\right]$. Since $\lim _{n \rightarrow \infty} y_{n}=1$ and $V$ is in the unit interval [0,1], $\lim _{n \rightarrow \infty} V\left(H_{n}\right)-V\left(H_{n}^{\prime}\right)=0$.

The next lemma establishes that $V$ has the expected utility property for all $H \in \mathbf{H}_{\mathbf{R}}$.

LEMMA 3.12. If $H_{a}, H_{b} \in \mathbf{H}_{\mathbf{R}}$, then $V\left(x H_{a}+(1-x) H_{b}\right)=x V\left(H_{a}\right)+$ $(1-x) V\left(H_{b}\right)$.

Proof. Consider two sequences $\left\{H_{a, n}\right\} \Rightarrow H_{a}$ and $\left\{H_{b, n}\right\} \Rightarrow H_{a}$, where each of $H_{a, n}$ and $H_{b, n}$ is simple and belongs to $\mathbf{H}_{\mathbf{R}}$. Then, for each $n$, the act $x\left(H_{a, n}\right)+(1-x) H_{b, n}$ is simple and belongs to $\mathbf{H}_{\mathbf{R}}$. It is evident that $\left\{x\left(H_{a, n}\right)+(1-x) H_{b, n}\right\} \Rightarrow x H_{a}+(1-x) H_{b}$. By Lemma 3.10, $V\left(x H_{a, n}+\right.$ $\left.(1-x) H_{b, n}\right)=x V\left(H_{a, n}\right)+(1-x) V\left(H_{b, n}\right)$. Then by Lemma 3.11, $V\left(x H_{a}+\right.$ $\left.(1-x) H_{b}\right)=x V\left(H_{a}\right)+(1-x) V\left(H_{b}\right)$.

Last, define the weak order $\precsim_{\omega}$ for $H \in \mathbf{H}_{\mathbf{R}}$ using the utilities fixed by $V$ :

Definition 27. ( $\left.H_{1} \precsim_{\omega} H_{2}\right)$ iff $\mathbf{V}\left(H_{1}\right) \leq \mathbf{V}\left(H_{2}\right)$.

We complete the proof of Theorem 3:

(i) That $\precsim_{\omega}$ is a weak order over elements of $\mathbf{H}_{\mathbf{R}}$ follows simply by noting that $V$ is real-valued. By Lemma 3.12, it satisfies the independence axiom. The Archimedean axiom also is a simple consequence of Lemmas 3.10 and 3.11 , that is, if $\left\{M_{n}\right\} \Rightarrow M,\left\{N_{n}\right\} \Rightarrow N$ and $M_{n} \prec_{\omega} N_{n}$, then $V(M) \leq V(N)$. Next, let $H_{a}$ and $H_{b}$ be simple, that is, each with finite support. Suppose $\left(H_{a} \prec H_{b}\right)$. According to Lemma 3.10 , the utilities $V\left(H_{a}\right)$ and $V\left(H_{b}\right)$ are determined by some stage $k$ of the induction, where $k$ is the maximum index of the (finitely many) elements of $\mathscr{H}$ in the combined supports of $H_{a}$ and $H_{b}$. Lemma 3.2 establishes that $\prec_{k}$ extends $\prec$. Then $\left(L_{1} \prec_{k} L_{2}\right)$ and thus $V\left(H_{a}\right)<V\left(H_{b}\right)$. Therefore, $\Im_{\omega}$ extends $\prec$ for simple lotteries.

(ii) We argue that $V$ almost agrees with $\prec$, that is, if $\left(H_{1} \prec H_{2}\right)$, then $\left(H_{1} \precsim_{V} H_{2}\right)$. Here is a simple lemma about the changing endpoints of target sets which completes the theorem.

LeMMA 3.13. For every act $H \in \mathbf{H}_{\mathbf{R}}$ and stage $j=2, \ldots$, (i) $v_{j-1 *}(H) \leq$ $v_{j *}(H) \leq v_{j}^{*}(H) \leq v_{j-1}^{*}(H)$ and (ii) $\lim _{j \rightarrow \infty} v_{j *}(H)=v_{j}^{*}(H)=V(H)$. 
Proof. (i) Since $\prec_{j}$ extends $\prec_{j-1}$, any sequence of $j-1$ stage preferences $H_{n} \prec_{j-1} x_{n} B+\left(1-x_{n}\right) W$ also obtain at stage $j$. Thus, by Definitions 20 and 25 and Lemma 3.2(i), $v_{j-1 *}(H) \leq v_{j *}(H) \leq v_{j}^{*}(H) \leq v_{j-1}^{*}(H)$.

(ii) For each act $\tilde{H}_{i} \in \mathscr{H}, \forall(j>i), v_{j *}\left(\tilde{H}_{i}\right)=v_{j}^{*}\left(\tilde{H}_{i}\right)=V\left(\tilde{H}_{i}\right)=v_{i}$. Hence, (ii) is obvious for all simple lotteries. Assume $H$ is not simple. It is easy to find a convergent sequence of simple acts in $\mathbf{H}_{\mathbf{R}},\left\{K_{n}\right\} \Rightarrow H$, where $v_{n *}\left(K_{n}\right)=v_{n}^{*}\left(K_{n}\right)=V\left(K_{n}\right)$ and $H=y_{n} K_{n}+\left(1-y_{n}\right) M_{n}$. The sequence of acts $M_{n}$, though elements of $\mathbf{H}_{\mathbf{R}}$, need not converge. Since $H$ is not simple, $y_{n}<1$. Then, $y_{n} K_{n}+\left(1-y_{n}\right) \mathbf{W} \prec y_{n} K_{n}+\left(1-y_{n}\right) M_{n} \prec y_{n} K_{n}+\left(1-y_{n}\right) \mathbf{B}$. As each $\prec_{n}$ extends $\prec$, we have $y_{n} V\left(K_{n}\right)<v_{n *}(H) \leq v_{n}^{*}(H)<y_{n} V\left(K_{n}\right)+$ $\left(1-y_{n}\right)$. However, $\lim _{n \rightarrow \infty} y_{n}=1$ and, by Lemma 3.8, $\lim _{n \rightarrow \infty} V\left(K_{n}\right)=V(H)$. Thus, $\lim _{j \rightarrow \infty} v_{j *}(H)=\lim _{j \rightarrow \infty} v_{j}^{*}(H)=V(H)$.

Finally, if $\left(H_{1} \prec H_{2}\right)$, since for each $n, \prec_{n}$ extends $\prec$, we have that $v_{n *}\left(H_{1}\right) \leq v_{n}^{*}\left(H_{2}\right)$. Then by Lemma 3.13, $V\left(H_{1}\right) \leq V\left(H_{2}\right)$.

\section{Other results from Section 3 .}

Proof of CoRollary 3.1. The extensions $\prec_{i}$ created in Theorem 3 rely on the existence at stage $i-1$ of a nonempty target set $\mathscr{T}_{i}\left(\tilde{H}_{i}\right)$, only for the acts $\tilde{H}_{i} \in \mathscr{H}$. However, $\mathscr{T}_{i}(\cdot)$ is defined on all of $\mathbf{H}_{\mathbf{R}}$, including the nonsimple acts. Hence, we can amend the sequence of extensions of $\prec$ to fix utilities for any countable set of acts, in addition to fixing utilities for each element of $\mathscr{H}$. Just modify the argument of Theorem 3 to assign utilities to the countable set $\mathscr{H} \cup \mathscr{B}$.

In connection with Example 3.1, for instance, we can introduce acts $H_{a}$ and $H_{b}$ into a well ordering of $\mathscr{H}$, for example, $\left\{\tilde{H}_{1}, H_{a}, \tilde{H}_{2}, H_{b}, \tilde{H}_{3}, \ldots\right\}$, so that by stage 4 of the sequence of extensions, $k_{1}=V\left(H_{a}\right)<V\left(H_{b}\right)=k_{2}$, which precludes the undesired limit stage in which $V\left(r_{i}\right)=0.25(i=1, \ldots)$.

Theorem 4 is easily demonstrated.

Proof OF Theorem 5. That $\phi \neq \mathscr{V} \subseteq \mathscr{Z}^{\mathscr{S}}$ is part (i) of Theorem 4. For the converse, argue indirectly. If $Z \in \mathscr{Z}^{\mathscr{S}} / \mathscr{V}$ then let $\tilde{H}_{k}$ be the first element of $\mathscr{H}$ (that is, let $k$ be the least integer) for which $Z\left(\tilde{H}_{k}\right) \notin \mathscr{T}_{k}\left(\tilde{H}_{k}\right)$, even though $v_{1}=Z\left(\tilde{H}_{1}\right), \ldots, v_{k-1}=Z\left(\tilde{H}_{k-1}\right)$ for acts $\tilde{H}_{1}, \ldots, \tilde{H}_{k-1}$. Then $Z$ agrees with $\prec_{k-1}$, since $\prec_{k-1}$ is the result of extending $\prec$ by the conditions $\tilde{H}_{i} \approx$ $v_{i} B+\left(1-v_{i}\right) W(i=1, \ldots, k-1)$. That is, expand each $\prec_{k-1}$-preference into a $\prec$-preference. The former follows from the latter by adding a set of $k-1$ assumptions $\left\{\tilde{H}_{i} \approx v_{i} B+\left(1-v_{i}\right) W:(i=1, \ldots, k-1)\right\}$ to $\prec$. But these $k-1$ conditions are satisfied under $Z$, and $Z$ agrees with $\prec$ on simple acts. Hence, it must be that either $Z\left(\tilde{H}_{k}\right)=v_{k}=v_{k *}\left(\tilde{H}_{k}\right)$ and $\mathscr{T}_{k}\left(\tilde{H}_{k}\right)$ is open at the lower end or else $Z\left(\tilde{H}_{k}\right)=v_{k}=v_{k}^{*}\left(\tilde{H}_{k}\right)$ and $\mathscr{T}_{k}\left(\tilde{H}_{k}\right)$ is open at the upper end. However, if the target set is open and if an endpoint $v_{k}$ of $\mathscr{T}_{k}\left(\tilde{H}_{k}\right)$ is not a 
candidate utility for $\tilde{H}_{k}$, then adding $\tilde{H}_{k} \approx v_{k} B+\left(1-v_{k}\right) W$ to $\prec_{k-1}$ produces a $\prec_{k-1}$-precluded preference. Since $Z$ agrees with $\prec_{k-1}, Z$ does not agree with any $\prec_{k-1}$-precluded preference. Thus, $Z$ cannot assign act $\tilde{H}_{k}$ the utility $v_{k}$, which contradicts the assumption $Z\left(\tilde{H}_{k}\right)=v_{k}$.

E. Results from Section 4. The proof of Lemma 4.1 is immediate after Theorem 13.1 of Fishburn (1979).

Proof of Lemma 4.2. Recall the strict preferences $\mathbf{W} \prec H \prec \mathbf{B}$, whenever $W, B \notin \operatorname{supp}(H)$. Hence, for each $V$, we may standardize the (expected) utility of act $\mathbf{W}$ as 0 and the (expected) utility of act $\mathbf{B}$ as 1 , where all other acts (not involving $W$ and $B$ ) have (expected) cardinal utilities in the open interval $(0,1)$. Next, define a set of simple, called-off acts $\left\{H_{i, j} \in \mathbf{H s}_{\mathbf{j}}\right\}$, which yield the lottery outcome $L_{i} \in \mathbf{L}_{\mathbf{R}-\{W, B\}}$ in state $s_{j}$ and outcome $W$ in all other states. In keeping with this notation, let $H_{W, j}=W$ and let $H_{B, j}$ be the $\mathbf{H s}_{\mathbf{j}}$ act with outcome $B$ in state $s_{j}$. Recall, for each $j, \lim _{m \rightarrow \infty}\left\{H_{m, j}^{i}\right\} \Rightarrow H_{i, j}$ and $H_{m, j}^{W}=H_{W, j}=\mathbf{W}$. Then, whenever $H_{L_{i}}<H_{L_{k}}$ (by HL Axiom 5), $\mathbf{W} \prec H_{m, j}^{i} \prec$ $H_{m, j}^{k} \prec H_{m, j}^{B}$. Hence, by the Archimedean HL Axiom 3 (as in Lemma 2.3), we have the restriction $\neg\left(H_{B, j} \prec H_{k, j} \prec H_{i, j} \prec \mathbf{W}\right)$. Moreover, this constraint obtains also for each extension of $\prec$, including all the limit extensions $\precsim_{V}$ since these $\prec$-preferences involve simple acts. Then, for each $V, \mathbf{W} \precsim_{V}$ $H_{k, j} \Im_{V} H_{i, j} \preccurlyeq_{V} H_{B, j}$. Trivially, either $\mathbf{W} \approx_{V} H_{B, j}$ or else $\mathbf{W} \prec_{V} H_{B, j}$. The upshot is that, for each $V$, one of two circumstances obtains:

Case 1. If $\mathbf{W} \approx_{V} H_{B, j}, H_{\alpha, j} \approx_{V} H_{\beta, j}$ and $s_{j}$ is null under $\lessgtr_{V}$, so $p\left(s_{j}\right)=0$.

Case 2. If $\mathbf{W} \prec_{V} H_{B, j}$, then $s_{j}$ is $V$-nonnull and for each representation of $V$ as an expected, state-dependent utility [in accord with condition (4.1)], $U_{j}(W) \leq U_{j}\left(L_{i}\right) \leq U_{j}\left(L_{k}\right) \leq U_{j}(B)$, with at least one of the outside inequalities strict. However, since the $U_{j}$ are defined only up to a similarity transformation, without loss of generality choose $U_{j}(W)=0$ and $U_{j}(B)=1$ and rescale $p$ accordingly.

Proof of Lemma 4.3. Without loss of generality (Corollary 3.3), let the denumerable sequence $\mathscr{H}=\left\{\tilde{H}_{i}\right\}$ of simple horse lotteries, used to create the set $\mathscr{V}$ of extensions for $\prec$, take $\left\{H_{r_{1}}, \ldots, H_{r_{n}}\right\}$ as its initial segment: the constant acts that award $r_{i}$ in each state. Suppose the interval $\mathscr{T}_{1}\left(r_{1}\right)$ is not open, for example, $\mathscr{T}_{1}\left(r_{1}\right)=\left[v_{1 *}, v_{1}^{*}\right)$. Then $0<v_{1 *}$. Extend $\prec$ according to the condition $H_{r_{1}} \approx_{1} v_{1 *} \mathbf{B}+\left(1-v_{1 *}\right) \mathbf{W}$. That is (by Definition 2.3), $H_{1} \prec_{1}$ $H_{2}$ iff $x H_{1}+(1-x) G_{1} \prec x H_{2}+(1-x) G_{1}^{\prime}$, where $G_{1}$ and $G_{1}^{\prime}$ are constant acts, symmetric mixtures of outcomes $r_{1}$ and $v_{1 *} B+\left(1-v_{1 *}\right) W$.

We show that each $V \in \mathscr{V}$ which extends $\prec_{1}$ (where $V$ is standardly represented by the set of pairs $\left\{\left(p, U_{j}\right)\right\}$ according to condition (4.1)) carries only state-independent utilities for $r_{1}$. That is, for each such $U_{j}, U_{j}\left(r_{1}\right)=v_{1} *$ if $s_{j}$ is $p$-nonnull. To verify this claim, define act $H_{j}$ as follows:

$H_{-\varepsilon, j}\left(s_{j}\right)=\left(v_{1 *}-\varepsilon\right) B+\left(1-\left[v_{1 *}-\varepsilon\right]\right) W$ and $H_{-\varepsilon, j}(s)=W \quad$ for $s \notin s_{j}$. If state $s_{j}$ is not $\prec$-potentially null then, since $v_{1 *}$ is the lower bound of $\mathscr{T}_{1}\left(r_{1}\right)$, by HL Axiom 4, we have $\forall\left(v_{1 *}>\varepsilon>0\right), H_{-\varepsilon, j} \prec H_{1, j}$. [Recall, 
$H_{1, j}\left(s_{j}\right)=r_{1}$ and $H_{1, j}(s)=W$ when $s \notin s_{j}$.] By the Archimedean condition HL Axiom 3, letting $\varepsilon \rightarrow 0$, we find that these $\prec$-preferences create the constraint $\neg\left(H_{1, j} \prec H_{\varepsilon=0, j}\right)$, which applies also to each extension of $\prec$. Thus, if $s_{j}$ is not $\prec$-potentially null, each $V$ which extends $\prec_{1}$ has $v_{1 *}$ as a lower bound on the state dependent utility $U_{j}\left(r_{1}\right)$. Likewise, by appeal to HL Axiom 5 in case $s_{j}$ is $\prec$-potentially null, it follows that $\neg\left(H_{1, j} \prec H_{\varepsilon=0, j}\right)$ and this applies also to all extensions of $\prec$. So, again, $v_{1 *}$ is a lower bound on the state dependent utility $U_{j}\left(r_{1}\right)$ for cases where $s_{j}$ is $\prec$-potentially null but $V$-nonnull and $\precsim_{V}$ extends $\prec$ (on simple acts). (Note: Here we use axiom HL Axiom 5 to regulate the state-dependent utility of lotteries in $\prec-$ potentially null states.) Because $V\left(r_{1}\right)=v_{1 *}$ for each $\precsim_{V}$ that extends $\prec_{1}$ on simple acts, $v_{1 *}$ also is an upper bound on all such $V$-nonnull statedependent utilities $U_{j}\left(r_{1}\right)$. This is so because $v_{1 *}=V\left(r_{1}\right)$ is the $p$-expectation of $U_{j}\left(r_{1}\right)$. Hence, each $\precsim_{V}$ that so extends $\prec_{1}$ assigns to reward $r_{1}$ the state-independent utility $v_{1 *}$.

Next, assume that $\mathscr{T}_{2}\left(r_{2}\right)$ is not an open interval, for example, let $\mathscr{T}_{2}\left(r_{2}\right)=$ $\left(v_{2 *}, v_{2}^{*}\right]$, and we know $v_{2}^{*}<1$. Thus, $H_{r_{2}} \prec_{1}\left(v_{2}^{*}+\varepsilon\right) \mathbf{B}+\left(1-\left[v_{2}^{*}+\varepsilon\right]\right) \mathbf{W}$. Extend $\prec_{1}$ to $\prec_{2}$ by introducing the $\approx_{2}$-condition $H_{r_{2}} \approx_{2} v_{2}^{*} \mathbf{B}+$ $\left(1-v_{2}^{*}\right) \mathbf{W}$. That is, define $\prec_{2}$ by $H_{1} \prec_{2} H_{2}$ iff $x H_{1}+(1-x) G_{2} \prec_{1} x H_{2}+$ $(1-x) G_{2}^{\prime}$, where $G_{2}$ and $G_{2}^{\prime}$ are constant horse lotteries, which are symmetric mixtures of acts $H_{r_{2}}$ and $v_{2}^{*} \mathbf{B}+\left(1-v_{2}^{*}\right) \mathbf{W}$.

To see that all $\lessgtr_{V}$-extensions of $\prec_{2}$ impose a state-independent utility on $r_{2}$, that is, to show $U_{j}\left(r_{2}\right)=v_{2}^{*}$, it suffices to demonstrate that $v_{2}^{*} B+$ $\left(1-v_{2}^{*}\right) W$ serves as an upper utility bound for $r_{2}$ over all $\prec_{1}, s_{j}$-called-off preferences, called-off if $s_{j}$ fails. In other words, we are to establish that, for each state $s_{j}$, the constraint $\neg\left(H_{v_{2}^{*}+\varepsilon, j} \prec_{1} H_{2, j}\right)$ applies to $\prec_{1}$ and its extensions. Then, by the reasoning we used above, since $V\left(r_{2}\right)=v_{2}^{*}$ for all $\lessgtr_{V}$ which extend $\prec_{2}$ (on simple acts), $v_{2}^{*}$ also is a lower utility bound for each state-dependent utility $U_{j}\left(r_{2}\right)$, and thus $U_{j}\left(r_{2}\right)$ is state-independent. That is, since $V\left(r_{2}\right)=v_{2}^{*}$ is the $p$-expectation of quantities, none of which is greater than $v_{2}^{*}$, then $U_{j}\left(r_{2}\right)=v_{2}^{*}$ if $P\left(s_{j}\right)>0$.

To establish that $v_{2}^{*}$ is such a state-independent upper bound, expand each of the relevant $\prec_{1}$-preferences, to wit, $\forall\left(1-v_{2}^{*}>\varepsilon>0\right)$ expand $H_{r_{2}} \prec_{1}\left(v_{2}^{*}+\varepsilon\right) \mathbf{B}+\left(1-\left[v_{2}^{*}+\varepsilon\right]\right) \mathbf{W}$, into its respective $\prec$-preference: $\exists x_{\varepsilon}>0, \exists\left(G_{1 \varepsilon}, G_{1 \varepsilon}^{\prime}\right)$,

$$
x_{\varepsilon} H_{r_{2}}+\left(1-x_{\varepsilon}\right) G_{1 \varepsilon} \prec x_{\varepsilon}\left[\left(v_{2}^{*}+\varepsilon\right) \mathbf{B}+\left(1-\left[v_{2}^{*}+\varepsilon\right]\right) \mathbf{W}\right]+\left(1-x_{\varepsilon}\right) G_{1 \varepsilon}^{\prime} .
$$

Each pair $\left(G_{1 \varepsilon}, G_{1 \varepsilon}^{\prime}\right)$ is a symmetric mixture of acts $H_{r_{1}}$ and $v_{1 *} \mathbf{B}+$ $\left(1-v_{1 *}\right) \mathbf{W}$. These $\prec$-preferences are between constant horse lottery acts. By appeal to HL Axiom 4 in case $s_{j}$ is not $\prec$-potentially null, or by appeal to HL Axiom 5 in case $s_{j}$ is $\prec$-potentially null, we arrive at a constraint for called-off acts involving the two lottery outcomes $x_{\varepsilon} r_{2}+\left(1-x_{\varepsilon}\right) G_{1 \varepsilon}$ and $x_{\varepsilon}\left[\left(v_{2}^{*}+\varepsilon\right) B+\left(1-\left[v_{2}^{*}+\varepsilon\right]\right) W\right]+\left(1-x_{\varepsilon}\right) G_{1 \varepsilon}^{\prime}$. Specifically, we obtain the restriction $\neg\left(H_{x+\varepsilon, j} \prec H_{x_{2}, j}\right)$ - a constraint on all extensions of $\prec-$ where

$$
H_{x+\varepsilon, j}\left(s_{j}\right)=x_{\varepsilon}\left[\left(v_{2}^{*}+\varepsilon\right) B+\left(1-\left[v_{2}^{*}+\varepsilon\right]\right) W\right]+\left(1-x_{\varepsilon}\right) G_{1 \varepsilon}^{\prime} \text { and }
$$




$$
\begin{aligned}
H_{x+\varepsilon, j}(s) & =W \quad \text { if } s \notin s_{j}, \\
H_{x_{2}, j}\left(s_{j}\right) & =x_{\varepsilon} r_{2}+\left(1-x_{\varepsilon}\right) G_{1 \varepsilon} \quad \text { and } \quad H_{x_{2}, j}(s)=W \quad \text { if } s \notin s_{j} .
\end{aligned}
$$

However, each $\precsim_{V}$ extension of $\prec_{1}$ (on simple acts) assigns to $r_{1}$ the state-independent utility $v_{1 *}$. Thus, each extension assigns $G_{1 \varepsilon}$ and $G_{1 \varepsilon}^{\prime}$ this same state-independent utility $v_{1 *}$. Then, as the constraint $\neg\left(H_{x+\varepsilon, j} \prec_{1}\right.$ $H_{x_{2}, j}$ ) obtains, so too does the constraint which results at the limit, when $\varepsilon=0$, and terms $G_{1 \varepsilon}$ and $G_{1 \varepsilon}^{\prime}$ are canceled according to HL Axiom 2. Hence, each $\precsim_{V}$ extension of $\prec_{1}$ has the quantity $v_{2}^{*}$ as an upper bound on the state-dependent utility $U_{j}\left(r_{2}\right)$ of $r_{2}$, provided $s_{j}$ is not null under $\precsim_{V}$. Therefore, since $V$ is a weighted average of $U_{j}$ values, $U_{j}\left(r_{2}\right)=v_{2}^{*}$ for each $\precsim_{V}$ that extends $\prec_{2}$ on simple acts.

Proceed, this way, through the first $n$ stages in the extension of $\prec$ (using Theorem 3), by choosing for the $i$ th stage either the condition $H_{r_{i}} \approx_{i} v_{i *} \mathbf{B}+$ $\left(1-v_{i *}\right) \mathbf{W}$ or the condition $H_{r_{i}} \approx_{i} v_{i}^{*} \mathbf{B}+\left(1-v_{i}^{*}\right) \mathbf{W}$, as $\mathscr{T}_{i}\left(r_{i}\right)$ is closed below or above (respectively). Then the set $\mathscr{V}^{\prime}$ of extensions for $\prec_{n}$ provides the requisite subset of $\mathscr{V}$. [Note: $\mathscr{V}^{\prime}$ may fail to be convex when $\mathscr{T}_{i}\left(r_{i}\right)$ is a closed interval, as in the example for Theorem 1. Then either endpoint may be chosen, but not values in between.]

F. Proof of Theorem 6. The proof of Theorem 6(i) is based on the idea of the proof of Lemma 4.3. The argument is by induction on the number of rewards, that is, on the length of the initial segment of $\left\{r_{1}, r_{2}, \ldots\right\}$. The method is a straightforward epsilon-delta technique of fixing the degree of state-dependence to be tolerated and then choosing target set values sufficiently close to a boundary of the target sets to force agreement with the allowed tolerance for state-dependent utilities.

The proof of Theorem 6(ii) follows the argument of Corollary 3.1; that is, use the countable set $\{\mathscr{R} \cup \mathscr{B}\}$ in forming the extensions of $\prec$, subject to the following modification in the ordering of $\{\mathscr{R} \cup \mathscr{B}\}$ : Fix $k$, which determines the initial segment of $\mathscr{R},\left\{r_{1}, \ldots, r_{k}\right\}$, over which the almost state-independent utilities are to be provided. Given a nonsimple act $H \in \mathscr{B}$, insert it into the sequence of extensions based on $\mathscr{H}$ only after these $k$-many rewards have been assigned their utilities. This method ensures that assigning utilities to the nonsimple acts in $\mathscr{B}$ does not interfere with using the boundary regions of the target sets of the $k$-many rewards, $\left\{r_{1}, \ldots, r_{k}\right\}$, to locate their almost state-independent utilities. For interesting discussion of this point, see Section 5 of Nau (1993).

Two remarks help to explain the content of Theorem 6. First, in light of Example 4.1, it may be that for each $\varepsilon>0$, $\prec$ admits an almost stateindependent utility, but (corresponding to $\varepsilon=0$ ) there is no agreeing probability/state-independent utility pair in the limit. That is, the limit (as $\varepsilon \rightarrow 0$ ) of the (nested) sets of agreeing, almost state-independent utilities is empty. Second, Definition 31 requires only that $\prec$ admit almost stateindependent utilities for each finite set of $n$-many rewards. Obviously, by 
increasing $n$, we can form sequences of (nested) sets of probability/utility pairs. However, Definition 31 does not provide for an almost state-independent utility covering infinitely many rewards simultaneously. We do not yet know whether, given our five axioms, there exists a nonempty limit (as $n \rightarrow \infty)$ to these nested sets.

\section{G. Results from Section 5 .}

Proof of Lemma 5.1. (i) By HL Axiom 2, $H_{1} \prec H_{2}$ iff $0.5 H_{1}+0.5 H_{1}^{\prime} \prec$ $0.5 H_{2}+0.5 H_{1}^{\prime}$. Regrouping terms on the r.h.s. of the second $\prec$ relation, we obtain $H_{1} \prec H_{2}$ iff $0.5 H_{1}+0.5 H_{1}^{\prime} \prec 0.5 H_{1}+0.5 H_{2}^{\prime}$. Another application of HL Axiom 2 yields the desired result: $H_{1} \prec H_{2}$ iff $H_{1}^{\prime} \prec H_{2}^{\prime}$.

(ii) Suppose $H_{1} \approx H_{2}$. By Corollary 2.3, it suffices to show that $x H_{1}^{\prime}+$ $(1-x) H_{3} \prec H_{4}$ iff $x H_{2}^{\prime}+(1-x) H_{3} \prec H_{4}$. By HL Axiom $2, x H_{1}^{\prime}+(1-x) H_{3}$ $\prec H_{4} \quad$ iff $z\left[x H_{1}^{\prime}+(1-x) H_{3}\right]+(1-z) H_{1} \prec z H_{4}+(1-z) H_{1} \quad(0<z \leq 1)$. Since $H_{1} \approx H_{2}$, by Corollary 2.3, substituting $H_{2}$ for $H_{1}$ on the l.h.s., the biconditional reads: iff $z\left[x H_{1}^{\prime}+(1-x) H_{3}\right]+(1-z) H_{2} \prec z H_{4}+(1-z) H_{1}$. Let $z x=1-z$, that is, $z=(1+x)^{-1}$. Then regrouping terms in $H_{1}^{\prime}$ and $H_{2}$, the biconditional reads: iff $z\left[x H_{2}^{\prime}+(1-x) H_{3}\right]+(1-z) H_{1} \prec z H_{4}+$ $(1-z) H_{1}$. Another application of HL Axiom 2 yields the desired result.

Proof of Theorem 8. Part (i) is immediate as $\mathbf{H}_{\mathbf{e}}$ is a subset of $\mathbf{H}_{\mathbf{R}}$. Specifically, if a weak order $\lessgtr_{V}$ (of Theorem 3) agrees with $\prec$, it agrees with $\prec_{\mathbf{e}}$. That is, consider the $e$-called-off family $\mathbf{H}_{\mathbf{e}}$, where $H(s)=W$ if $s \notin e$ and $\prec_{\mathbf{e}}$ is the restriction of $\prec$ to $\mathbf{H}_{\mathbf{e}}$. Let $H_{1}$ and $H_{2}$ be simple acts that belong to $\mathbf{H}_{\mathbf{e}}$. If $H_{1} \prec_{\mathbf{e}} H_{2}$, then $H_{1} \prec H_{2}$ and therefore $V\left(H_{1}\right) \prec V\left(H_{2}\right)$. Let the expected utility $V$ be given by the probability/(state-dependent) utility pair $\left(p, U_{j}\right)$. As $U_{j}(W)=0$ and $H_{i}(s)=W$ for $s \notin e(i=1,2)$, then $\sum_{s_{j} \in e} p\left(s_{j}\right) U_{j}\left(L_{1 j}\right)<\sum_{s_{j} \in e} p\left(s_{j}\right) U_{j}\left(L_{2 j}\right)$. Hence, $\left(p_{e}, U_{j \in e}\right)$ agrees with $\prec_{\mathbf{e}}$.

For part (ii), without loss of generality (Lemma 5.1), continue with the $e$-called-off family $\mathbf{H}_{\mathbf{e}}$ determined by fixing $H(s)=W$ if $s \notin e$. Define the act $\mathbf{B}_{\mathbf{e}} \in \mathbf{H}_{\mathbf{e}}$ by $\mathbf{B}_{\mathbf{e}}(s)=B$ if $s \in e$. With respect to $\prec_{\mathbf{e}}, \mathbf{B}_{\mathbf{e}}$ serves as the "best" act and $\mathbf{W}$ serves as the "worst." Thus, for $H \in \mathbf{H}_{\mathbf{e}}, V(H \mid e) V\left(\mathbf{B}_{\mathbf{e}}\right)=V(H)$. Let $V_{\mathbf{e}}(\cdot)$ agree with $\prec_{\mathbf{e}}$ over the set $\mathbf{H}_{\mathbf{e}}$. Assume $V_{\mathbf{e}}(\cdot)$ differs from each conditional expected utility $V(\cdot \mid e)(V \in \mathscr{V})$. In particular, with $\mathscr{H}_{\mathbf{e}}$ ordered for applying Theorem 3 to $\prec_{\mathbf{e}}$, let $H_{z} \in \mathscr{H}_{\mathbf{e}}$ satisfy the following condition: For each $V \in \mathscr{V}$ such that $V_{\mathbf{e}}\left(H_{i}\right)=V\left(H_{i} \mid e\right)(i=1, \ldots, z-1), V_{\mathbf{e}}\left(H_{z}\right) \neq V\left(H_{z} \mid e\right)$. That is, $H_{z}$ is the first $e$-called-off act, where $V_{\mathbf{e}}$ differs from each $V(\cdot \mid e)$, $V \in \mathscr{V}$. Without loss of generality, according to Corollary 3.3, put the first $z$-elements of $\mathscr{H}_{\mathbf{e}}$ as the initial segment of $\mathscr{H}$. Thus, $H_{z}$ is the $z$ th element in this reordering of $\mathscr{H}$.

By hypothesis, for some $V \in \mathscr{V}, V_{\mathbf{e}}\left(H_{i}\right)=V\left(H_{i} \mid e\right)(i=1, \ldots, z-1)$. Then mimic the first $z-1$ extensions of $\prec_{e}$ in the first $z-1$ extensions of $\prec$. That is, provided $e$ is not potentially null so that $\mathbf{W} \prec \mathbf{B}_{\mathbf{e}}$, use Definition 20 to extend $\prec$ to $\prec_{z-1}$ with symmetric mixtures of the $z-1$ act pairs: $H_{i}$ and $V_{\mathbf{e}}\left(H_{i}\right) \mathbf{B}_{\mathbf{e}}+\left(1-V_{\mathbf{e}}\left(H_{i}\right)\right) \mathbf{W}$. Also by hypothesis, $\prec_{z-1}$ cannot be ex- 
tended to $\prec_{z}$ using Definition 20 with symmetric mixtures of $H_{z}$ and $V_{\mathbf{e}}\left(H_{z}\right) \mathbf{B}_{\mathbf{e}}+\left(1-V_{\mathbf{e}}\left(H_{z}\right)\right) \mathbf{W}$.

Next, we show that $V_{\mathbf{e}}\left(H_{z}\right)$ is an endpoint of the conditional target set $\mathscr{T}_{z}\left(H_{z}\right)$, defined using mixtures of $\mathbf{B}_{\mathbf{e}}$ and $\mathbf{W}$. Argue indirectly: either $H_{z} \prec_{z-1}$ $V_{\mathbf{e}}\left(H_{z}\right) \mathbf{B}_{\mathbf{e}}+\left(1-V_{\mathbf{e}}\left(H_{z}\right)\right) \mathbf{W}$ or else $V_{\mathbf{e}}\left(H_{z}\right) \mathbf{B}_{\mathbf{e}}+\left(1-V_{\mathbf{e}}\left(H_{z}\right)\right) \mathbf{W} \prec_{z-1} H_{z}$. We give the analysis for the former case. (The reasoning for the latter case is parallel.) Expand the $\prec_{z-1}$-preference into its equivalent $\prec$-preference. Thus, for $i=1, \ldots, z-1$, there exist $x_{i} \geq 0, x_{z}>0, \sum_{i} x_{i}+x_{z}=1$, such that

$$
\begin{aligned}
x_{1} G_{1} & +\cdots+x_{z-1} G_{z-1}+x_{z} H_{z} \\
& \prec x_{1} G_{1}^{\prime}+\cdots+x_{z-1} G_{z-1}^{\prime}+x_{z}\left[V_{\mathbf{e}}\left(H_{z}\right) \mathbf{B}_{\mathbf{e}}+\left(1-V_{\mathbf{e}}\left(H_{z}\right)\right) \mathbf{W}\right],
\end{aligned}
$$

where the pairs $\left(G_{i}, G_{i}^{\prime}\right)$ are symmetric mixtures of $H_{i}$ and $V_{\mathbf{e}}\left(H_{i}\right) \mathbf{B}_{\mathbf{e}}+$ $\left(1-V_{\mathbf{e}}\left(H_{i}\right)\right) \mathbf{W}$. However, as this $\prec$-preference involves elements of $\mathscr{C}_{\mathbf{e}}$ only, then $x_{1} G_{1}+\cdots+x_{z-1} G_{z-1}+x_{z} H_{z} \prec_{\mathbf{e}} x_{1} G_{1}^{\prime}+\cdots+x_{z-1} G_{z-1}^{\prime}+x_{z}\left[V_{\mathbf{e}}\left(H_{z}\right)\right.$ $\left.\times \mathbf{B}_{\mathbf{e}}+\left(1-V_{\mathbf{e}}\left(H_{z}\right)\right) \mathbf{W}\right]$. Thus $V_{\mathbf{e}}\left(H_{z}\right) \notin \mathscr{T}_{\mathbf{e}, z}\left(H_{z}\right)$ - a contraction with the assumption that $V_{\mathbf{e}}(\cdot)$ agrees with $\prec_{\mathbf{e}}$. Hence, $V_{\mathbf{e}}\left(H_{z}\right)$ is a precluded endpoint of the conditional $\mathscr{T}_{z}\left(H_{z}\right)$ according to preferences $\prec_{z-1}$, but it is not precluded from $\mathscr{T}_{\mathbf{e}, z}\left(H_{z}\right)$ according to the subset of preferences in $\prec_{\mathbf{e}, z-1}$.

Acknowledgments. The authors thank Peter Fishburn for his careful reading of an earlier version of this paper and for providing us with insightful and extensive feedback. Also, we thank Robert Nau for stimulating exchanges relating to matters of state-dependent utility. We are exceedingly grateful to the two Annals referees, whose patient and detailed readings of our long manuscript resulted in many helpful ideas and useful suggestions.

\section{REFERENCES}

Anscombe, F. J. and Aumann, R. J. (1963). A definition of subjective probability. Ann. Math. Statist. 34 199-205.

Aumann, R. J. (1962). Utility theory without the completeness axiom. Econometrica $30445-462$.

Aumann, R. J. (1964). Utility theory without the completeness axiom: a correction. Econometrica 32 210-212.

Berger, J. (1985). Statistical Decision Theory and Bayesian Analysis, 2nd ed. Springer, New York.

DE FinetTi, B. (1937). La prevision: ses lois logiques, ses sources subjectives. Ann. Inst. H. Poincaré 7 1-68.

DeGroot, M. (1974). Reaching a consensus. J. Amer. Statist. Assoc. 69 118-121.

EllsberG, D. (1961). Risk, ambiguity, and the Savage axioms. Quart. J. Econom. 75 643-669.

FishbuRn, P. C. (1979). Utility Theory for Decision Making. Krieger, New York.

FishbuRn, P. C. (1982). The Foundations of Expected Utility. Reidel, Dordrecht.

Giron, F. J. and RIos, S. (1980). Quasi-Bayesian behaviour: A more realistic approach to decision making? In Bayesian Statistics (J. M. Bernardo, M. H. DeGroot, D. V. Lindley and A. F. M. Smith, eds.) 17-38. Univ. Valencia Press.

Hartigan, J. A. (1983). Bayes Theory. Springer, New York.

Hausner, M. (1954). Multidimensional utilities. In Decision Processes (R. M. Thrall, C. H. Coombs and R. L. Davis, eds.) 167-180. Wiley, New York. 
KaDANE, J. B., Ed. (1984). Robustness of Bayesian Analysis. North-Holland, Amsterdam.

KADANE, J. B. (1986). Progress toward a more ethical method for clinical trials. Journal of Medicine and Philosophy 11 385-404.

KadAne, J. B. and Sedransk, N. (1980). Toward a more ethical clinical trial. In Bayesian Statistics (J. M. Bernardo, M. H. DeGroot, D. V. Lindley and A. F. M. Smith, eds.) 329-338. Univ. Valencia Press.

KANNAI, Y. (1963). Existence of a utility in infinite dimensional partially ordered spaces. Israel J. Math. 1 229-234.

LEVI, I. (1974). On indeterminate probabilities. J. Philos. 71 391-418.

LEVI, I. (1980). The Enterprise of Knowledge. MIT Press.

LeVI, I. (1990). Pareto unanimity and consensus. J. Philos. 87 481-492.

Moskowitz, H., Wong, R. T. and CHU, P.-Y. (1988). Robust interactive decision-analysis (RID): Concepts, methodology, and system principles. Paper 948, Krannert Graduate School of Management, Purdue Univ.

NAU, R. F. (1992). Indeterminate probabilities on finite sets. Ann. Statist. 20 1737-1767.

NAU, R. F. (1993). The shape of incomplete preferences. Paper 9301, The Fuqua School of Business, Duke Univ.

Ramsey, F. P. (1931). Truth and probability. In The Foundations of Mathematics and Other Logical Essays (R. B. Braithwaite, ed.) 156-198. Kegan, Paul, Trench, Trubner and Co. Ltd., London.

Rios Insua, D. (1990). Sensitivity Analysis in Multi-Objective Decision Making. Springer, New York.

Rios InsuA, D. (1992). On the foundations of decision making under partial information. Theory and Decision 33 83-100.

Savage, L. J. (1954). The Foundations of Statistics. Wiley, New York.

Schervish, M. J., Seidenfeld, T. and KadAne, J. B. (1990). State-dependent utilities. J. Amer. Statist. Assoc. 85 840-847.

Schervish, M. J., Seidenfeld, T. and Kadane, J. B. (1991). Shared preferences and statedependent utilities. Management Sci. 37 1575-1589.

Seidenfeld, T., Kadane, J. B. and Schervish, M. J. (1989). On the shared preferences of two Bayesian decision makers. J. Philos. 86 225-244.

Seidenfeld, T. and Schervish, M. J. (1983). A conflict between finite additivity and avoiding Dutch Book. Philos. Sci. 50 398-412.

Seidenfeld, T., Schervish, M. J. and Kadane, J. B. (1990). Decisions without ordering. In Acting and Reflecting (W. Sieg, ed.) 143-170. Kluwer, Dordrecht.

Sмiтн, C. A. B. (1961). Consistency in statistical inference and decision. J. Roy. Statist. Soc. Ser. B 23 1-25.

SzPIlRaJn, E. (1930). Sur l'extension de l'ordre partiel. Fund. Math. 16 386-389.

von Neumann, J. and Morgenstern, O. (1947). Theory of Games and Economic Behavior, 2nd ed. Princeton Univ. Press.

Walley, P. (1991). Statistical Reasoning with Imprecise Probabilities. Chapman and Hall, London.

White, C. C. (1986). A posteriori representations based on linear inequality descriptions of a priori and conditional probabilities. IEEE Trans. Systems Man Cybernet. 16 570-573.

Williams, P. (1976). Indeterminate probabilities. In Formal Methods in the Methodology of Empirical Sciences (M. Przelecki, K. Szaniawski and R. Wojcicki, eds.) 229-246. Reidel, Dordrecht.

Departments of Philosophy and Statistics Carnegie Mellon University

PitTsburgh, Pennsylvania 15213 